TOPICAL REVIEW

\title{
Spin photocurrents in quantum wells
}

\author{
S D Ganichev and W Prettl
}

Fakultät für Physik, Universität Regensburg, D-93040 Regensburg, Germany

Received 10 February 2003

Published 12 May 2003

Online at stacks.iop.org/JPhysCM/15/R935

\begin{abstract}
Spin photocurrents generated by homogeneous optical excitation with circularly polarized radiation in quantum wells (QWs) are reviewed. The absorption of circularly polarized light results in optical spin orientation due to the transfer of the angular momentum of photons to electrons of a two-dimensional electron gas. It is shown that in QWs belonging to one of the gyrotropic crystal classes a non-equilibrium spin polarization of uniformly distributed electrons causes a directed motion of electrons in the plane of the QW. A characteristic feature of this electric current, which occurs in unbiased samples, is that it reverses its direction upon changing the radiation helicity from left-handed to right-handed and vice versa.

Two microscopic mechanisms are responsible for the occurrence of an electric current linked to a uniform spin polarization in a QW: the spin polarization-induced circular photogalvanic effect and the spin-galvanic effect. In both effects the current flow is driven by an asymmetric distribution of spin-polarized carriers in $\boldsymbol{k}$-space of systems with lifted spin degeneracy due to $k$-linear terms in the Hamiltonian. Spin photocurrents provide methods to investigate spin relaxation and to reach a conclusion as regards the inplane symmetry of QWs. The effect can also be utilized to develop fast detectors for determining the degree of circular polarization of a radiation beam. Furthermore, spin photocurrents under infrared excitation were used to demonstrate and investigate monopolar spin orientation of free carriers.
\end{abstract}

\section{Contents}

1. Introduction 936

2. Homogeneous spin orientation-induced photocurrents 938

2.1. Removal of spin degeneracy 938

2.2. The circular photogalvanic effect $\quad 942$

2.3. The spin-galvanic effect 948

2.4. The spin orientation-induced circular photogalvanic effect versus the spingalvanic effect 952 
3. Methods 953

3.1. Samples 953

3.2. The experimental technique 955

4. Experimental results and discussion 956

4.1. The spin polarization-induced circular photogalvanic effect 956

4.2. The spin-galvanic effect 961

4.3. Monopolar spin orientation 968

4.4. The spin-controlled non-linearity of the spin orientation-induced CPGE 969

5. Spin-independent photocurrents under homogeneous excitation 971

$\begin{array}{ll}\text { 5.1. The linear photogalvanic effect } & 973\end{array}$

5.2. The photon drag effect 975

6. Spin photocurrents caused by inhomogeneities 977

$\begin{array}{ll}\text { 7. Summary } & 978\end{array}$

$\begin{array}{ll}\text { Acknowledgments } & 979\end{array}$

$\begin{array}{ll}\text { References } & 979\end{array}$

\section{Introduction}

The spin of electrons and holes in solid-state systems is an intensively studied quantum mechanical property showing a large variety of interesting physical phenomena. Lately, there has been much interest in the use of the spin of carriers in semiconductor heterostructures together with their charge to realize novel concepts such as spintronics and several schemes of quantum computation (for a review see [1]). The necessary conditions for realizing spintronic devices are high spin polarizations in low-dimensional structures and large spin splitting of subbands in $\boldsymbol{k}$-space due to $\boldsymbol{k}$-linear terms in the Hamiltonian. The latter is important for the ability to manipulate spins with an external electric field by the Rashba effect [2]. Significant progress has been achieved recently in the injection of spin-polarized electrons (or holes), in demonstrating the Rashba splitting, and also in using the splitting for manipulating the spins [1].

One of the most frequently used and powerful methods of generation and investigation of spin polarization is optical orientation [3]. Optical generation of an unbalanced spin distribution in a semiconductor may lead to a spin photoconductive effect as well as to a spin photocurrent. In the spin photoconductive effect the optical spin orientation yields a change of conductivity which, upon application of an external voltage bias, results in a spin-polarized current [4-6]. On the other hand, spin photocurrents reviewed here occur without an external bias. They are electric currents which are driven by optically generated spin polarization.

A spin photocurrent was proposed for the first time in [7] (see also [8]) and thereafter observed in bulk AlGaAs [9]. In these works it was shown that an inhomogeneity of the spin polarization of electrons results in a surface current due to spin-orbit interaction. A gradient of spin density was obtained by making use of the strong fundamental absorption of circularly polarized light at the band edge of the semiconductor. This and other spin photocurrents caused by inhomogeneous spin orientation will be briefly outlined in section 6 .

In the present article we review a new property of the electron spin in a homogeneous spin-polarized two-dimensional electron gas: its ability to drive an electric current if some general symmetry requirements are met. Recently it was demonstrated that an optical excitation of quantum well $(\mathrm{QW})$ structures with circularly polarized radiation leads to a current whose direction and magnitude depend on the degree of circular polarization of the incident light [10]. This effect belongs to the class of photogalvanic effects which were intensively studied in semiconductors (for a review see [11-14]) and represents a circular photogalvanic 
effect (CPGE). The CPGE can be considered as a transfer of the photon angular momentum into a directed motion of a free charge carrier. It is an electronic analogue of mechanical systems which transmit rotatory motion into a linear one, such as a screw thread or a propeller. The CPGE was independently predicted by Ivchenko and Pikus [15] and Belinicher [16] and then observed in bulk tellurium [17, 18] (for reviews see [13, 14]). In tellurium the current arises due to spin splitting of the valence band edge at the boundary of the first Brillouin zone ('camel back' structure) [19]. While neither bulk zinc-blende structure materials such as GaAs and related compounds nor bulk diamond structure crystals such as $\mathrm{Si}$ and Ge allow this effect, in QW structures a CPGE is possible due to a reduction of symmetry.

It was shown in [20] that in zinc-blende structure-based QW structures a CPGE is caused by optical spin orientation of carriers in systems with band splitting in $\boldsymbol{k}$-space due to $\boldsymbol{k}$-linear terms in the Hamiltonian. Here $\boldsymbol{k}$ is the two-dimensional electron wavevector in the plane of the QW. In this case homogeneous irradiation of QWs with circularly polarized light results in a non-uniform distribution of photoexcited carriers in $\boldsymbol{k}$-space due to optical selection rules and energy and momentum conservation, which leads to a current [20-26]. The carrier distribution in the real space remains uniform.

Furthermore, a thermalized but spin-polarized electron gas can drive an electrical current $[27,28]$. Recently it was demonstrated that a homogeneous spin polarization obtained by any means, not necessarily optical, yields a current, if the same symmetry requirements as allow $\boldsymbol{k}$-linear terms in the Hamiltonian are met [29]. This phenomenon is referred to as the spin-galvanic effect. While electrical currents are usually generated by electric or magnetic fields or gradients, in this case a uniform non-equilibrium population of electron spins gives rise to an electric current. The microscopic origin of the spin-galvanic effect is an inherent asymmetry of spin-flip scattering of electrons in systems with removed $\boldsymbol{k}$-space spin degeneracy of the band structure. This effect has been demonstrated by optical spin orientation [29-31] and therefore also represents a spin photocurrent.

Both the spin orientation-induced CPGE and the spin-galvanic effect in QWs occur upon one-photon excitation yielding an electric charge current linked to a spin polarization. However, a pure spin current may be obtained upon simultaneous one- and two-photon coherent excitation of appropriate polarization as recently demonstrated for bulk GaAs [32, 33]. This phenomenon may be attributed to a photogalvanic effect where the reduced symmetry is caused by the coherent two-frequency excitation [34] which may also occur in QWs [35].

Spin photocurrents occurring upon homogeneous excitation have been observed in $\mathrm{n}$ - and $\mathrm{p}$-type QWs based on various semiconductor materials for very different types of optical excitation achieved by application of several lasers at wavelengths ranging from the visible to the far-infrared. There is particular interest in spin photocurrents generated by infrared radiation because, in contrast to the case for conventional methods of optical spin orientation using inter-band transitions [3], only one type of charge carrier is excited yielding monopolar spin orientation [29, 36-41]. Therefore infrared spin orientation allows one to study spin relaxation without electron-hole interaction and exciton formation under conditions close to the case for electrical spin injection [29, 38, 39]. Finally, spin photocurrents have found technical application as room temperature detectors which allow one to determine and monitor the state of polarization of terahertz radiation with picosecond time resolution [42].

This article is organized in the following way. In section 2 an overview of mechanisms yielding photocurrents for homogeneous spin orientation in QWs is given. First the removal of spin degeneracy due to spin-orbit interaction in QWs is discussed and then it is shown that spin splitting in $\boldsymbol{k}$-space is the basic reason for the different mechanisms of spin photocurrents in QWs. Section 3 gives a short account of the experimental technique. In section 4 the experimental results are presented and discussed on the basis of the theoretical background. 
Section 5 sketches several kinds of spin-independent photocurrent in comparison to spin photocurrents, and experimental methods are introduced allowing one to distinguish between spin-dependent and spin-independent currents. Finally, in section 6 we present several mechanisms of spin generation due to inhomogeneities.

\section{Homogeneous spin orientation-induced photocurrents}

Light propagating through a semiconductor and acting upon mobile carriers can generate a dc electric current without external bias. The irradiated sample represents a current source. Here we consider photocurrents which appear due to optically induced homogeneous spin orientation of carriers in homogeneous samples. The microscopic origin of these currents is the conversion of spin polarization of carriers into directed motion. The fingerprint of spin photocurrents is their dependence on the helicity of the radiation field. The current reverses its direction by switching the polarization of light from right-handed circular to left-handed circular and vice versa. The experimental data can be described by simple analytical expressions derived from a phenomenological theory which shows that the effect can only be present in gyrotropic media. This requirement rules out effects depending on the helicity of the radiation field in bulk nonoptically active materials such as bulk zinc-blende structure and diamond structure crystals. The reduction of dimensionality as realized in QWs makes spin photocurrents possible. The effect is quite general and has so far been observed in GaAs [10, 20, 29, 38], InAs [20, 29, 30], BeZnMnSe [43] QW structures, and in asymmetric SiGe QWs [23].

On a microscopical level, spin photocurrents are the result of spin orientation in systems with $k$-linear terms in the Hamiltonian which may also occur only in gyrotropic media. In general, two mechanisms contribute to spin photocurrents: photoexcitation and scattering of photoexcited carriers. The first is the spin orientation-induced CPGE which is caused by the asymmetry of the momentum distribution of carriers excited in optical transitions which are sensitive to the light circular polarization due to selection rules [20]. The second mechanism is the spin-galvanic effect which is a result of spin relaxation. In general this effect does not need optical excitation but may also occur due to optical spin orientation [29]. The current caused by the CPGE is spin polarized and decays with the momentum relaxation time of free carriers, whereas the spin-galvanic effect-induced current is unpolarized but decays with the spin relaxation time.

\subsection{Removal of spin degeneracy}

2.1.1. $k$-linear terms in the effective Hamiltonian. Quantum phenomena in semiconductors are highly sensitive to subtle details of the carrier energy spectrum, so even a small spin splitting of energy bands may result in measurable effects. Spin-dependent terms linear in the wavevector $\boldsymbol{k}$ in the effective Hamiltonian remove the spin degeneracy in $\boldsymbol{k}$-space of the carrier spectrum. The presence of these terms in QWs gives rise to spin photocurrents, yields beating patterns in Shubnikov-de Haas oscillations [2, 44, 45], determines spin relaxation in QWs [1, 46, 47], results in spin-polarized tunnelling [48-51], and allows the control of spin orientation by external fields [1, 2, 52-58].

In the general case, the terms linear in $k$ appear because the symmetry of heterostructures is lower than the symmetry of the corresponding bulk materials. Spin degeneracy of electron bands in semiconductors and subbands of heterostructures results because of the simultaneous presence of time reversal and spatial inversion symmetry. In the present case of lowdimensional heterostructures and QWs, the spatial inversion symmetry is broken. However, in order to obtain spin photocurrents depending on the helicity of radiation and spin orientation, 
inversion asymmetry is a necessary, but not a sufficient condition. In fact, the materials must belong to one of the gyrotropic crystal classes which have second-rank pseudo-tensors as invariants. As a consequence, spin-dependent $\boldsymbol{k}$-linear terms caused by spin-orbit interaction appear in the electron Hamiltonian leading to a splitting of electronic subbands in $\boldsymbol{k}$-space. As long as the time reversal symmetry is not broken by the application of an external magnetic field, the degeneracy of Kramers doublets is not lifted, so still $\varepsilon(\boldsymbol{k}, \uparrow)=\varepsilon(-\boldsymbol{k}, \downarrow)$. Here $\varepsilon$ is the electron energy and the arrows indicate the spin orientation.

The principal sources of $k$-linear terms in the band structure of QWs are the bulk inversion asymmetry (BIA) of zinc-blende structure crystals and possibly a structural inversion asymmetry (SIA) of the low-dimensional quantizing structure (see [1, 2, 23, 4680] and references therein). In addition, an interface inversion asymmetry (IIA) may yield $\boldsymbol{k}$-linear terms caused by non-inversion symmetric bonding of atoms at heterostructure interfaces [1, 23, 81-87]. BIA induces $\boldsymbol{k}$-linear terms in the 2D Hamiltonian, known as Dresselhaus terms, due to the absence of an inversion centre in the bulk crystal. The Dresselhaus terms originate from the $\boldsymbol{k}$-cubic terms in the Hamiltonian of a bulk material [88]. Averaging these cubic terms along the quantization axis in the case of low subband filling with carriers gives rise to the terms linear in $\boldsymbol{k}$. These terms are present in QWs based on zinc-blende structure material and are absent in SiGe heterostructures. IIA may occur in zinc-blende structure-based QWs [81-86] where the well and the cladding have different compositions of both anions and cations, such as in InAs/GaSb QWs as well as in SiGe [23, 87]. IIA yields BIA-like terms in the effective Hamiltonian [23, 87]; thus on a phenomenological level a separation between BIA and IIA is not necessary.

The SIA contribution to the removal of spin degeneracy is caused by the intrinsic heterostructure asymmetry which need not be related to the crystal lattice. These $k$-linear terms in the Hamiltonian were first recognized by Rashba and are called Rashba terms [2, 89]. SIA may arise from different kinds of asymmetry of the heterostructures such as non-equivalent normal and inverted interfaces, asymmetric doping of QWs, asymmetrically shaped QWs, and external or built-in electric fields, and may also exist in QWs prepared from materials with inversion symmetry such as $\mathrm{Si}$ and $\mathrm{Ge}[23,86]$. It is the SIA term which allows control of the spin polarization by externally applied electric fields [2]. Therefore these spin-orbit coupling terms are important for spintronics and in particular for the spin transistor [90].

In the unperturbed symmetric case, we will assume a doubly degenerate subband. Then the spin-orbit coupling in the non-symmetric structure has the form

$$
\hat{H}^{\prime}=\sum_{l m} \beta_{l m} \sigma_{l} k_{m}
$$

where $\beta_{l m}$ is a second-rank pseudo-tensor and $\sigma_{l}$ are the Pauli matrices. The Pauli matrices occur here because of time reversal symmetry.

In equation (1), BIA, IIA, and SIA can be distinguished by decomposing $\sigma_{l} k_{m}$ into a symmetric and an anti-symmetric product:

$$
\sigma_{l} k_{m}=\left\{\sigma_{l}, k_{m}\right\}+\left[\sigma_{l}, k_{m}\right]
$$

with the symmetric term

$$
\left\{\sigma_{l}, k_{m}\right\}=\frac{1}{2}\left(\sigma_{l} k_{m}+\sigma_{m} k_{l}\right)
$$

and the anti-symmetric term

$$
\left[\sigma_{l}, k_{m}\right]=\frac{1}{2}\left(\sigma_{l} k_{m}-\sigma_{m} k_{l}\right) .
$$

Now the perturbation can be written as

$$
\hat{H}^{\prime}=\sum_{l m}\left(\beta_{l m}^{s}\left\{\sigma_{l} k_{m}\right\}+\beta_{l m}^{a}\left[\sigma_{l} k_{m}\right]\right)
$$


where $\beta_{l m}^{s}$ and $\beta_{l m}^{a}$ are symmetric and anti-symmetric pseudo-tensors projected out of the full tensor by the symmetric and anti-symmetric products of $\sigma_{l} k_{m}$, respectively. The symmetric term describes BIA as well as possible IIA terms, whereas the anti-symmetric term is caused by SIA.

2.1.2. Spin splitting of energy bands in zinc-blende structure-based $Q W s$. The pseudo-tensor $\beta_{l m}$ as a material property must transform following the identity representation of the point group symmetry of the QW. The point group is determined by the crystallographic orientation and the profile of growth and doping of the QWs. The three point groups $\mathrm{D}_{2 \mathrm{~d}}, \mathrm{C}_{2 \mathrm{v}}$, and $\mathrm{C}_{\mathrm{s}}$ are particularly relevant for zinc-blende structure-based QWs [14]. Hereafter, the Schönflies notation is used to label the point groups. In the international notation they are labelled as $\overline{4} 2 m, m m 2$, and $m$, respectively. The $\mathrm{D}_{2 \mathrm{~d}}$ point group symmetry corresponds to perfectly grown (001)-oriented QWs with symmetric doping. In such QWs only BIA and IIA terms may exist. The symmetry of (001)-grown QWs reduces from $D_{2 d}$ to $C_{2 v}$ if an additional asymmetry is present due to e.g. non-equivalent interfaces, asymmetric growth profiles, and asymmetric doping, resulting in SIA. The relative strength of BIA, IIA, and SIA depends on the structure of the QW. In structures of strong growth direction asymmetry such as heterojunctions, the SIA term may be larger than the BIA and IIA terms. The last point group is $\mathrm{C}_{\mathrm{s}}$, which contains only two elements: the identity and one mirror reflection plane. It is realized for instance in (113)-oriented and miscut (001)-oriented samples.

The non-zero components of the pseudo-tensor $\beta_{l m}$ depend on the symmetry and the coordinate system used. For (001) crystallographic orientation-grown QWs of $D_{2 \mathrm{~d}}$ and $\mathrm{C}_{2 \mathrm{v}}$ symmetry, the tensor elements are given in the coordinate system $(x y z)$ with $x \|[1 \overline{10}$, $y \|$ [110], $z \|$ [001]. The coordinates $x$ and $y$ are in the reflection planes of both point groups perpendicular to the principal twofold axis; $z$ is along the growth direction normal to the plane of the QW. In $\mathrm{D}_{2 \mathrm{~d}}$ the pseudo-tensor $\beta_{l m}$ is symmetric, $\beta_{l m}=\beta_{l m}^{s}$. In the above coordinate system there are two non-zero components, $\beta_{x y}$ and $\beta_{y x}$ with $\beta_{y x}=\beta_{x y}=\beta_{y x}^{s}$. For zinc-blende structure-type crystals, it has been shown that the BIA and IIA terms in the Hamiltonian have the same form; thus, IIA enhances or reduces the strength of the BIA-like term.

Therefore we obtain ${ }^{1}$

$$
\hat{H}^{\prime}=\hat{H}_{B I A}+\hat{H}_{I I A}=\beta_{x y}^{s}\left(\sigma_{x} k_{y}+\sigma_{y} k_{x}\right) .
$$

In $\mathrm{C}_{2 \mathrm{v}}$ the tensor $\beta_{l m}$ is non-symmetric, yielding additional terms in $\hat{H}^{\prime}$ caused by SIA, so now $\hat{H}^{\prime}=\hat{H}_{B I A}+\hat{H}_{I I A}+\hat{H}_{S I A}$. The form of $\hat{H}_{B I A}$ and $\hat{H}_{I I A}$ remains unchanged by the reduction of symmetry from $\mathrm{D}_{2 \mathrm{~d}}$ to $\mathrm{C}_{2 \mathrm{v}}$. The SIA term in $\mathrm{C}_{2 \mathrm{v}}$ assumes the form

$$
\hat{H}_{S I A}=\beta_{x y}^{a}\left(\sigma_{x} k_{y}-\sigma_{y} k_{x}\right) .
$$

It is clear that the form of this term is independent of the orientation of cartesian coordinates in the plane of the QW. The strength of spin splitting was experimentally derived e.g. from beatings of Shubnikov-de Haas oscillations in various III-V compound-based QWs [2, 44, 45]. It has been found to be in the range of $10^{-10}$ to $10^{-9} \mathrm{eV} \mathrm{cm}^{-1}$ and was attributed to SIA.

The point group $\mathrm{C}_{\mathrm{s}}$ is discussed using the example of (113) orientation-grown QWs because they are available and spin because the photocurrents in them have already been intensively investigated $^{2}$. In this case, we use the coordinates $x^{\prime}=x \|[1 \overline{10}]$, as above, $y^{\prime} \|$ [332] $], z^{\prime} \|$ [113]. Direction $x$ is normal to the reflection plane, the only non-identity

1 For coordinates along cubic axes, $x \|[100]$ and $y \|[010]$, we have non-zero components $\beta_{x x}$ and $\beta_{y y}$ with $\beta_{y y}=-\beta_{x x}$ which yields $\hat{H}^{\prime}=\hat{H}_{B I A}+\hat{H}_{I I A}=\beta_{x x}\left(\sigma_{x} k_{x}-\sigma_{y} k_{y}\right)$.

${ }_{2}$ The miscut (001)-oriented samples investigated in [10] also have $\mathrm{C}_{\mathrm{s}}$ symmetry. 
(a)

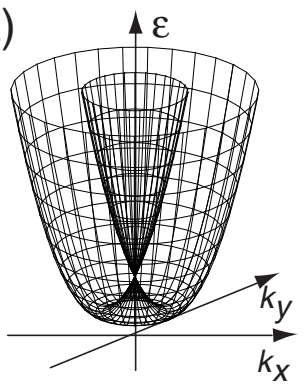

(c)

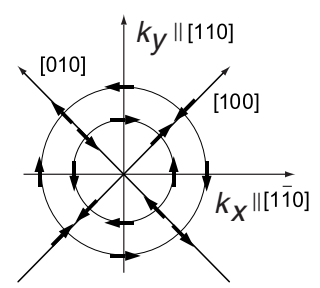

(d)

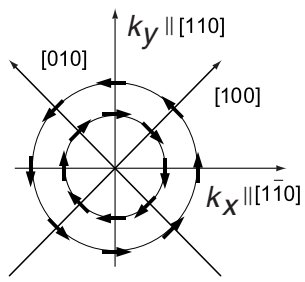

(b)

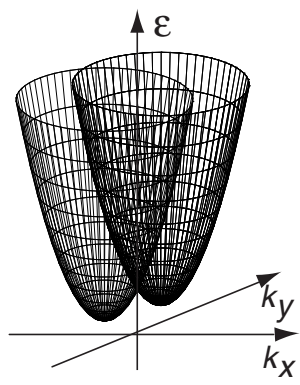

(e)

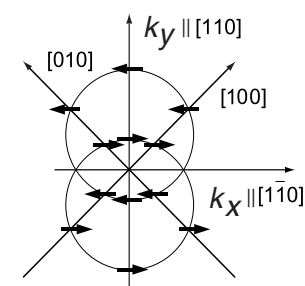

$\mathrm{BIA}=\mathrm{SIA}$ (f)

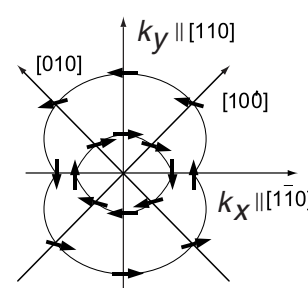

$\mathrm{BIA} \neq \mathrm{SIA}$ $\mathrm{SIA}=0$

Figure 1. A schematic 2D band structure with $k$-linear terms for $\mathrm{C}_{2 \mathrm{v}}$ symmetry. The energy $\varepsilon$ is plotted as a function of $k_{x}$ and $k_{y}$ in (a) with only one type of inversion asymmetry, BIA or SIA, respectively, and in (b) for equal strengths of the BIA and SIA terms in the Hamiltonian. The bottom plate shows the distribution of spin orientations at the 2D Fermi energy for different strengths of the BIA and SIA terms.

symmetry element of this group, and $z^{\prime}$ is along the growth direction. The reduction of the symmetry to $\mathrm{C}_{\mathrm{s}}$ results in an additional term in the Hamiltonian:

$$
\hat{H}^{\prime}=\beta_{z^{\prime} x} \sigma_{z^{\prime}} k_{x} \text {. }
$$

In order to illustrate band structures with a $\boldsymbol{k}$-linear term in figure 1, we plotted the energy $\varepsilon$ as a function of $k_{x}$ and $k_{y}$ for $\mathrm{C}_{2 \mathrm{v}}$ symmetry. The upper plate of figure 1 shows the band structure with only one type of inversion asymmetry, BIA or SIA (figure 1(a)), and the band structure at equal strength of the BIA (including IIA) and SIA (figure 1(b)). In the illustration we assume positive coefficients $\beta_{l m}^{a}, \beta_{l m}^{s} \geqslant 0$. In the case of BIA only $\left(\beta_{l m}^{a}=0\right)$ or SIA only $\left(\beta_{l m}^{s}=0\right)$, the band structure is the result of the revolution around the energy axis of two parabolas symmetrically displaced with respect to $k=0$. A constant-energy surface is a pair of concentric circles; however, the spins are oriented differently for BIA and SIA. The distribution of spin orientation in $k$-space, obtained by the procedure of [68], is indicated by arrows in the bottom plate of figure 1. The distribution of spins for a pure BIA term is shown in figure 1(c). If only the SIA term is present (figure 1(d)), then the spins are always oriented normal to the wavevector $\boldsymbol{k}$. This is a consequence of the vector product in the Rashba spinorbit interaction [2]. If the strengths of BIA and SIA are the same, then the 2D band structure consists of two revolution paraboloids with revolution axes symmetrically shifted in opposite directions with respect to $k=0$ (figure 1(b)). Now the spins are oriented along $\pm k_{x}$ as shown in figure 1(e). In figure 1(f) we have shown a constant-energy surface and the direction of spins for $\beta_{l m}^{a} \neq \beta_{l m}^{s}$. 
(a)

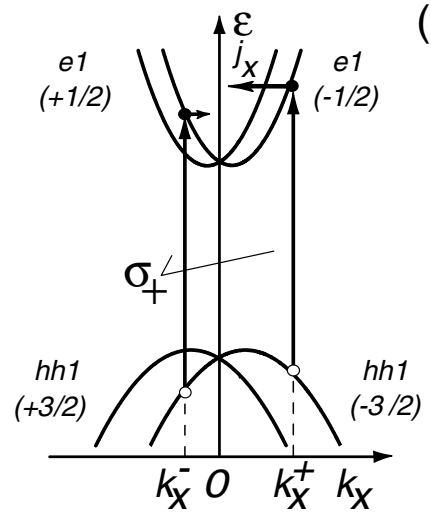

(b)

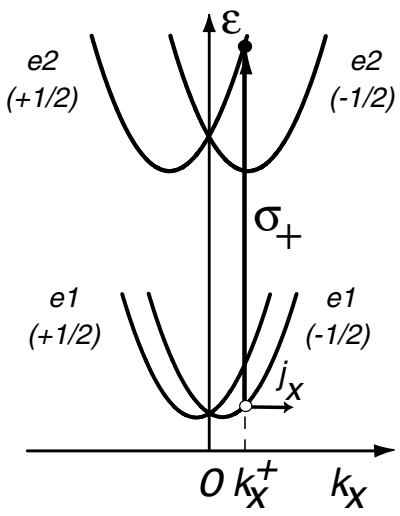

Figure 2. A microscopic picture of the spin orientation-induced CPGE for direct transitions in the $\mathrm{C}_{\mathrm{s}}$ point group taking into account the splitting of subbands in $k$-space. $\sigma_{+}$-excitation induces direct transitions (solid arrows) (a) between the valence and conduction band (from hh1 $\left(m_{s}=-3 / 2\right)$ to e1 $\left.\left(m_{s}=-1 / 2\right)\right)$ and (b) between size-quantized subbands in the conduction band (from e1 $\left(m_{s}=-1 / 2\right)$ to $\left.2\left(m_{s}=+1 / 2\right)\right)$. Spin splitting together with optical selection rules results in an unbalanced occupation of the positive $\left(k_{x}^{+}\right)$and negative $\left(k_{x}^{-}\right)$states yielding a spin-polarized photocurrent. For $\sigma_{-}$-excitation both the spin orientation of the charge carriers and the current direction get reversed. In each plate, arrows indicate the current due to an imbalance of carriers. Currents are shown for one subband only.

Finally, we briefly discuss QWs prepared on SiGe. As both Si and Ge possess inversion centres, there is no BIA; however, both IIA, with a BIA-like form of the Hamiltonian, and SIA may lead to $\boldsymbol{k}$-linear terms $[23,79,86,87]$. The symmetry of $\mathrm{Si} /\left(\mathrm{Si}_{1-x} \mathrm{Ge}_{x}\right)_{n} / \mathrm{Si} \mathrm{QW}$ depends on the number $n$ of monatomic layers in the well. In the case of (001) crystallographic orientation-grown QW structures with an even number $n$, the symmetry of QWs is $\mathrm{D}_{2 \mathrm{~h}}$ which is inversion symmetric and does not yield $\boldsymbol{k}$-linear terms. An odd number of $n$, however, interchanges the [1 $\overline{10}$ ] and [110] axes of the adjacent barriers and reduces the symmetry to $\mathrm{D}_{2 \mathrm{~d}}$ with the same implication as treated above for zinc-blende structure QWs [23].

\subsection{The circular photogalvanic effect}

2.2.1. The microscopic model. Inter-band transitions: The spin orientation-induced CPGE is most easily conceivable for both $\mathrm{n}$ - and $\mathrm{p}$-type materials from the schematic band structure shown in figure 2(a) [20]. We assume direct inter-band transitions in a $\mathrm{QW}$ of $\mathrm{C}_{\mathrm{s}}$ symmetry. For the sake of simplicity we take into account a one-dimensional band structure consisting only of the lowest conduction subband $\mathrm{e} 1$ and the highest heavy-hole subband hh1. The splitting in the conduction band is given by $\varepsilon_{e 1, \pm 1 / 2}(k)=\left[\left(\hbar^{2} k_{x}^{2} / 2 m_{e 1}\right) \pm \beta_{e 1} k_{x}+\varepsilon_{g}\right]$ and that in the valence band by $\varepsilon_{h h 1, \pm 3 / 2}(\boldsymbol{k})=-\left[\left(\hbar^{2} k_{x}^{2} / 2 m_{h h 1}\right) \pm \beta_{h h 1} k_{x}\right]$, where $\varepsilon_{g}$ is the energy gap.

For absorption of circularly polarized radiation of photon energy $\hbar \omega$, energy and momentum conservation allow transitions only for two values of $k_{x}$. By the selection rules, the optical transitions occur from $m_{s}=-3 / 2$ to $-1 / 2$ for right-handed circular polarization $\left(\sigma_{+}\right)$ and from $m_{s}=3 / 2$ to $1 / 2$ for left-handed circular polarization $\left(\sigma_{-}\right)$. Here the $m_{s}$ are the spin quantum numbers of the electron states. The corresponding transitions for e.g. $\sigma_{+}$-photons occur at

$$
k_{x}^{ \pm}=+\frac{\mu}{\hbar^{2}}\left(\beta_{e 1}+\beta_{h h 1}\right) \pm \sqrt{\frac{\mu^{2}}{\hbar^{4}}\left(\beta_{e 1}+\beta_{h h 1}\right)^{2}+\frac{2 \mu}{\hbar^{2}}\left(\hbar \omega-\varepsilon_{g}\right)},
$$

and are shown in figure 2(a) by the solid vertical arrows. Here $\mu=\left(m_{e 1} m_{h h 1}\right) /\left(m_{e 1}+m_{h h 1}\right)$ 
is a reduced mass. The 'centre of mass' of these transitions is shifted from the point $k_{x}=0$ by $\left(\beta_{e 1}+\beta_{h h 1}\right)\left(\mu / \hbar^{2}\right)$. Thus the sum of the electron velocities in the excited states in the conduction band, $v_{e 1}=\hbar\left(k_{x}^{-}+k_{x}^{+}-2 k_{x}^{\text {min }}\right) / m_{e 1}=2 /\left[\hbar\left(m_{e 1}+m_{h h 1}\right)\right]\left(\beta_{h h 1} m_{h h 1}-\beta_{e 1} m_{e 1}\right)$, is non-zero. The contributions of $k_{x}^{ \pm}$-photoelectrons to the current do not cancel each other except in the case of $\beta_{e 1} m_{e 1}=\beta_{h h 1} m_{h h 1}$ which corresponds to equal splittings of the conduction and the valence band. We note that the group velocity is obtained when it is taken into account that the $k_{x}^{ \pm}$are to be counted from the conduction subband minima $k_{x}^{\min }$ because the current is caused by the difference of the group velocities within the subband. The same consideration applies for holes in the initial states in hh1. Consequently, a spin-polarized net current in the $x$ direction results. Changing the circular polarization of the radiation from $\sigma_{+}$to $\sigma_{-}$reverses the current because the 'centre of mass' of these transitions is now shifted to $-\left(\beta_{e 1}+\beta_{h h 1}\right)\left(\mu / \hbar^{2}\right)$.

Inter-subband transitions: In the longer-wavelength range, infrared or far infrared, the current is caused by inter-subband or intra-subband transition. For direct transition between size-quantized states in the valence or conduction band, for example e1 and e 2 in n-type materials, the model is very similar to that of the inter-band transitions discussed above [24]. In figure 2(b) we sketch this situation for QWs of $\mathrm{C}_{\mathrm{s}}$ symmetry. The contribution $\sigma_{z^{\prime}} k_{x}$ to the Hamiltonian splits the electron spectrum into spin sublevels with the spin components $m_{s}= \pm 1 / 2$ along the growth direction $z^{\prime}$. As a result of optical selection rules, right-handed circular polarization under normal incidence induces direct optical transitions between the subband e1 with spin $m_{s}=-1 / 2$ and 22 with spin $m_{s}=+1 / 2$. For monochromatic radiation, optical transitions occur only at a fixed $k_{x}^{+}$, where the energy of the incident light matches the transition energy, as is indicated by the arrow in figure 2(b). Therefore optical transitions induce an imbalance of momentum distribution in the two subbands yielding an electric current in the $x$-direction with contributions from e1 and e2. As in n-type QWs the energy separation between 1 and 2 is typically larger than the energy of longitudinal optical phonons $\hbar \omega_{L O}$, the non-equilibrium distribution of electrons in e 2 relaxes rapidly due to emission of phonons. As a result, the contribution of the e 2 subband to the electric current vanishes. Thus the magnitude and the direction of the current are determined by the group velocity and the momentum relaxation time $\tau_{p}$ of photogenerated holes in the initial state of the resonant optical transition in the e 1 subband with $m_{s}=-1 / 2$.

Intra-subband transitions (Drude absorption): Now we consider indirect intra-subband transitions. This situation is usually realized in the far-infrared range where the photon energy is not high enough to excite direct inter-subband transitions. Due to energy and momentum conservation, intra-subband transitions can only occur by absorption of a photon and simultaneous absorption or emission of a phonon. This process is described by virtual transitions involving intermediate states and will be discussed in more detail in section 4.3.2. It can be shown that transitions via intermediate states within one and the same subband do not yield spin orientation and do not contribute to the spin photocurrent. However, spinselective indirect optical transitions excited by circularly polarized light with both initial and final states in the conduction band can generate a spin current if virtual processes involve intermediate states in different subbands [20]. Figure 3 sketches the underlying mechanism for $\sigma_{+}$-polarization. For the sake of simplicity, only the spin splitting of the valence band is taken into account. The two virtual transitions shown represent excitations which, for $\sigma_{+}$-helicity, transfer electrons from states with negative $k_{x}$ to states with positive $k_{x}$. The current resulting from a free electron transition (solid arrow) in the conduction band e 1 occurs due to transitions involving intermediate states in the valence subbands. Two representative virtual transitions for $\sigma_{+}$-excitation are illustrated in figure 3 . One is an optical transition from $m_{s}=+1 / 2$ to $+3 / 2$ (dashed line, downward arrow) and a transition involving a phonon from $m_{s}=+3 / 2$ back to the conduction band (dash-dotted line, upward arrow). The other is a phonon transition 


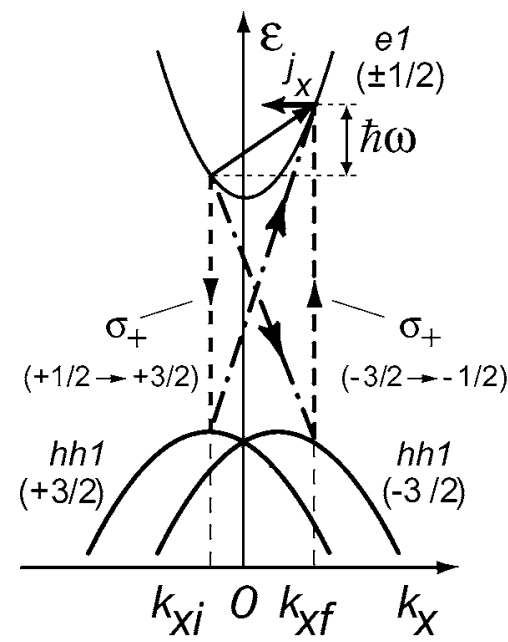

Figure 3. A microscopic picture of the origin of the spin orientation-induced CPGE for indirect (Drude) transitions in $\mathrm{C}_{\mathrm{s}}$ point group samples.

from the conduction band to the $m_{s}=-3 / 2$ intermediate state in hh1 and an optical transition from $m_{s}=-3 / 2$ to $-1 / 2$. While the first route depopulates preferentially initial states of spin $m_{s}=+1 / 2$ for $k_{x i}<0$, the second one populates preferentially final state of $m_{s}=-1 / 2$ states for $k_{x f}>0$ [20]. This, together with the unbalanced occupation of the $k$-space, causes a spin-polarized photocurrent. Switching the helicity from $\sigma_{+}$to $\sigma_{-}$reverses the process and results in a spin photocurrent in the opposite direction.

2.2.2. Phenomenology. On the macroscopic level the CPGE can be described by the following phenomenological expression [14]:

$$
\begin{aligned}
& j_{\lambda}=\sum_{\mu} \gamma_{\lambda \mu} \mathrm{i}\left(\boldsymbol{E} \times \boldsymbol{E}^{*}\right)_{\mu}, \\
& \mathrm{i}\left(\boldsymbol{E} \times \boldsymbol{E}^{*}\right)_{\mu}=\hat{e}_{\mu} E_{0}^{2} P_{\text {circ }}
\end{aligned}
$$

where: $\boldsymbol{j}$ is the photocurrent density; $\gamma$ is a second-rank pseudo-tensor; $\boldsymbol{E}$ is the complex amplitude of the electric field of the electromagnetic wave; $E_{0}, P_{\text {circ }}, \hat{\boldsymbol{e}}=\boldsymbol{q} / q$, and $\boldsymbol{q}$ are the electric field amplitude, the degree of circular polarization, the unit vector pointing in the direction of light propagation, and the light wavevector inside the medium, respectively. The photocurrent is proportional to the radiation helicity $P_{\text {circ }}$ and can be observed only under circularly polarized excitation. The helicity of the incident radiation is given by

$$
P_{\text {circ }}=\frac{I_{\sigma_{+}}-I_{\sigma_{-}}}{I_{\sigma_{+}}+I_{\sigma_{-}}}=\sin 2 \varphi
$$

where $\varphi$ is the phase angle between the $x$ - and $y$-components of the electric field vector. $P_{\text {circ }}$ varies from -1 (left-handed circular, $\sigma_{-}$) to +1 (right-handed circular, $\sigma_{+}$).

In general, in addition to the circular photogalvanic current given in equation (10), two other photocurrents can be simultaneously present, namely the linear photogalvanic effect (LPGE) and the photon drag effect. Both effects were observed in low-dimensional structures. They do not require spin orientation and will be summarized in section 5 .

As a result of tensor equivalence the second-rank pseudo-tensor $\gamma$ is subjected to the same symmetry restriction as $\boldsymbol{\beta}$ responsible for the $\boldsymbol{k}$-linear terms in the Hamiltonian, discussed in 


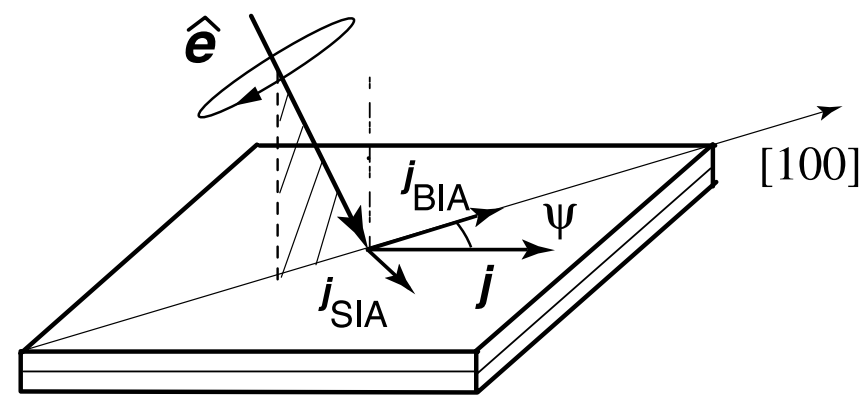

Figure 4. The SIA- and BIA-induced CPGE generated in samples of $\mathrm{C}_{2 \mathrm{v}}$ symmetry under oblique incidence of circularly polarized light with excitation along [100].

detail above. Thus, $\gamma_{l m}$ depends in the same way as $\beta_{l m}$ on the symmetry and the coordinate system. In the various crystal classes of importance here, the same elements of $\beta_{l m}$ as of $\gamma_{l m}$ are non-zero.

In the following we analyse equation (10) for $\mathrm{D}_{2 \mathrm{~d}}, \mathrm{C}_{2 \mathrm{v}}$, and $\mathrm{C}_{\mathrm{s}}$ in the coordinate systems $(x y z)$ and $\left(x y^{\prime} z^{\prime}\right)$. Due to carrier confinement in the growth direction, the photocurrent in QWs has non-vanishing components only in the plane of a QW.

For the point group $\mathrm{D}_{2 \mathrm{~d}}$ the non-zero components of $\gamma$ are $\gamma_{x y}$ and $\gamma_{y x}$ with $\gamma_{x y}=\gamma_{y x}$. We denote the only independent element by $\gamma^{(0)}=\gamma_{x y}$; then the current in a QW is given by

$$
j_{x}=\gamma^{(0)} \hat{e}_{y} E_{0}^{2} P_{\text {circ }}, \quad j_{y}=\gamma^{(0)} \hat{e}_{x} E_{0}^{2} P_{\text {circ }}
$$

where $E_{0}^{2}$ is the square of the electric field amplitude in vacuum, proportional to the radiation power $P$.

Equations (13) shows that in this configuration we get a transverse effect if the sample is irradiated along a $\langle 110\rangle$ crystallographic orientation, corresponding to $\hat{e}_{x}=1, \hat{e}_{y}=0$ or $\hat{e}_{x}=0, \hat{e}_{y}=1$. The current $j$ is perpendicular to the direction of light propagation $\hat{e}$. If the radiation is shone in along a cubic axis $\langle 100\rangle$, with $\hat{e}_{x}=\hat{e}_{y}=1 / \sqrt{2}$, then the current is longitudinal, flowing along the same cubic axis because $j_{x}=j_{y}$. Putting all of this together, we see from equations (13) that rotating $\hat{e}$ in the plane of the QW anticlockwise yields a clockwise rotation of $j$.

Reducing the symmetry from $\mathrm{D}_{2 \mathrm{~d}}$ to $\mathrm{C}_{2 \mathrm{v}}$, the tensor $\gamma$ describing the CPGE is characterized by two independent components $\gamma_{x y}$ and $\gamma_{y x} \neq \gamma_{x y}$. We define $\gamma^{(1)}=\gamma_{x y}$ and $\gamma^{(2)}=\gamma_{y x}$; then the photocurrent is determined by

$$
j_{x}=\gamma^{(1)} \hat{e}_{y} E_{0}^{2} P_{\text {circ }}, \quad j_{y}=\gamma^{(2)} \hat{e}_{x} E_{0}^{2} P_{\text {circ }} .
$$

If $\hat{e}$ is along $\langle 110\rangle$ with the result that $\hat{e}_{x}=1$ and $\hat{e}_{y}=0$ or $\hat{e}_{x}=0$ and $\hat{e}_{y}=1$, then the current again flows normal to the light propagation direction. In contrast to the case for $\mathrm{D}_{2 \mathrm{~d}}$ symmetry, the strengths of the current are different for the radiation propagating along $x$ and $y$. This is due to the non-equivalence of the crystallographic axes [110] and [110] because of the twofold rotation axis in $\mathrm{C}_{2 \mathrm{v}}$ symmetry. If the sample is irradiated with $\hat{e}$ parallel to $\langle 100\rangle$ corresponding to $\hat{e}_{x}=\hat{e}_{y}=1 / \sqrt{2}$, the current is neither parallel nor perpendicular to the light propagation direction (see figure 4 ). The current includes an angle $\psi$ with the $x$-axis given by $\tan \psi=\gamma_{x y} / \gamma_{y x}=\beta_{y x} / \beta_{x y}$. The last equation follows from tensor equivalence.

Another conclusion from equations (13) and (14) is that in QWs of the higher symmetries $\mathrm{D}_{2 \mathrm{~d}}$ and $\mathrm{C}_{2 \mathrm{v}}$ the photocurrent can only be induced under oblique incidence of irradiation. For normal incidence, $\hat{e}$ is parallel to [001] and hence the current vanishes as $\hat{e}_{x}=\hat{e}_{y}=0$. In contrast to this result, in QWs of $\mathrm{C}_{\mathrm{s}}$ symmetry a photocurrent also occurs for normal incidence 
of the radiation on the plane of the QW because the tensor $\gamma$ has an additional component $\gamma_{x z^{\prime}}$. The current here is given by

$$
j_{x}=\left(\gamma_{x y^{\prime}} \hat{e}_{y^{\prime}}+\gamma_{x z^{\prime}} \hat{e}_{z^{\prime}}\right) E_{0}^{2} P_{\text {circ }}, \quad j_{y^{\prime}}=\gamma_{y^{\prime} x} \hat{e}_{x} E_{0}^{2} P_{c i r c} .
$$

At normal incidence, $\hat{e}_{x}=\hat{e}_{y^{\prime}}=0$ and $\hat{e}_{z^{\prime}}=1$, the current in the QW flows perpendicular to the mirror reflection plane of $\mathrm{C}_{\mathrm{s}}$ which corresponds to the $x$-coordinate parallel to [1 $\overline{1} 0$ ].

Now we will take a closer look at the dependence of the photocurrent on the angle of incidence $\Theta_{0}$ which is determined by the value of the projection of $\hat{e}$ on the $x$-axis or $y$-axis (see equations (13) and (14)) or on the $z^{\prime}$-axis (equations (15)). We have for the excitation in the plane of incidence parallel to $(y z)$

$$
\hat{e}_{x}=t_{p} t_{s} \sin \Theta,
$$

and in the plane of incidence parallel to $\left(y^{\prime} z^{\prime}\right)$

$$
\hat{e}_{z^{\prime}}=t_{p} t_{s} \cos \Theta,
$$

where $\Theta$ is the refraction angle defined by $\sin \Theta=\sin \Theta_{0} / \sqrt{\varepsilon^{*}}, \varepsilon^{*}$ is the dielectric constant of the QW material, and the transmission coefficients $t_{p}, t_{s}$ for linear $\mathrm{p}$ and s polarizations are given, using Fresnel's formula, by

$$
t_{p} t_{s}=\frac{4 \cos ^{2} \Theta_{0}}{\left(\cos \Theta_{0}+\sqrt{\varepsilon^{*}-\sin ^{2} \Theta_{0}}\right)\left(\varepsilon^{*} \cos \Theta_{0}+\sqrt{\varepsilon^{*}-\sin ^{2} \Theta_{0}}\right)} .
$$

For $\hat{e}_{y^{\prime}}$ in the left equation of equations (15) we obtain $\hat{e}_{y^{\prime}}=t_{p} t_{s} \sin \Theta$ for the excitation in the plane of incidence parallel to $\left(y^{\prime} z\right)$.

The measurement of the CPGE with respect to the angle of incidence and the crystallographic direction is important for determining the in-plane symmetry of the QW. Indeed, only in $\mathrm{C}_{\mathrm{s}}$ symmetry does the CPGE occur at normal incidence (see equations (15)), and $\mathrm{D}_{2 \mathrm{~d}}$ and $\mathrm{C}_{2 \mathrm{v}}$ symmetries may be distinguished by excitation along a $\langle 100\rangle$ axis, because in this case only $\mathrm{D}_{2 \mathrm{~d}}$ does not allow a transverse effect.

2.2.3. Microscopic theory. Inter-band transitions: The microscopic theory of the spin orientation-induced CPGE in QWs was worked out for inter-band excitation in $[21,25,26]$ and is briefly sketched here following [21]. We consider the asymmetry of the momentum distribution of holes excited under direct inter-band optical transitions in p-doped (113)-grown QWs of $\mathrm{C}_{\mathrm{s}}$ symmetry. We recall that in this case normally incident radiation of circular polarization induces a current in the $x$-direction. Let us denote the free hole states in a $\mathrm{QW}$ as $\left|v m_{s} \boldsymbol{k}\right\rangle$, where $v$ and $m_{s}$ are the hole subband and spin branch indices, respectively. If only terms even in $k$ are taken into account in the effective Hamiltonian of holes, all hole subbands $(v, k)$ are doubly degenerate. Allowing terms odd in $\boldsymbol{k}$ in the Hamiltonian results in a subband spin splitting, so the hole energy $\varepsilon_{v m_{s} k}$ becomes dependent on the spin branch index $m_{s}$. The photocurrent density is given by a standard expression

$$
j_{x}=e \sum_{\nu m_{s} k} u_{x}\left(v m_{s} \boldsymbol{k}\right) f_{\nu m_{s} k}
$$

where $e$ is the elementary charge (for holes $e>0), u_{x}\left(v m_{s} k\right)$ is the group velocity $\hbar^{-1}\left(\partial \varepsilon_{v m_{s} k} / \partial k_{x}\right)$, and $f_{v m_{s} k}$ is the non-equilibrium steady-state distribution function. Note that the energy $\varepsilon_{v m_{s} k}$ is invariant and the velocity $u_{x}\left(v m_{s} k\right)$ changes its sign under the time inversion operation $K$ transforming a spinor $\hat{\psi}$ into $K \hat{\psi} \equiv \mathrm{i} \sigma_{y} \hat{\psi}$ ( $\sigma_{y}$ is one of the Pauli matrices) [14]. Therefore only the anti-symmetric part of the distribution function $f_{v m_{s} k}^{-}=\left(f_{v m_{s} k}-f_{v \bar{m}_{s},-k}\right) / 2$ contributes to $j_{x}$. Here $\left|\nu \bar{m}_{s},-\boldsymbol{k}\right\rangle$ is obtained from $\left|\nu m_{s} \boldsymbol{k}\right\rangle$ by application of the operator $K$. 
In the momentum relaxation time approximation under direct optical transitions we have

$$
j_{x}=e \sum_{v^{\prime} v m_{s}^{\prime} m_{s} k} W_{\nu^{\prime} m_{s}^{\prime}, v m_{s}}(\boldsymbol{k}, \boldsymbol{e})\left[u_{x}\left(v^{\prime} m_{s}^{\prime} \boldsymbol{k}\right) \tau_{p}^{\left(\nu^{\prime}\right)}-u_{x}\left(v m_{s} \boldsymbol{k}\right) \tau_{p}^{(v)}\right]
$$

where $e$ is the light polarization unit vector and $\tau_{p}^{(v)}$ is the hole momentum relaxation time in the subband $v$. The probability rate for the transition $\left|v m_{s} \boldsymbol{k}\right\rangle \rightarrow\left|v^{\prime} m_{s}^{\prime} \boldsymbol{k}\right\rangle$ is given by Fermi's golden rule

$W_{\nu^{\prime} m_{s}^{\prime}, v m_{s}}(\boldsymbol{k}, \boldsymbol{e})=\frac{2 \pi}{\hbar}\left|M_{\nu^{\prime} m_{s}^{\prime}, v m_{s}}(\boldsymbol{k})\right|^{2}\left(f_{\nu m_{s} k}^{0}-f_{\nu^{\prime} m_{s}^{\prime} k}^{0}\right) \delta\left(\varepsilon_{\nu^{\prime} m_{s}^{\prime} k}-\varepsilon_{\nu m_{s} k}-\hbar \omega\right)$,

where $M_{\nu^{\prime} m_{s}^{\prime}, v m_{s}}(\boldsymbol{k})$ is the inter-subband optical matrix element proportional to the amplitude of the electromagnetic field $E_{0}$ and $f_{v m_{s} k}^{0}$ is the distribution function in equilibrium. For the sake of simplicity, it is assumed that the light intensity is low enough to allow us to ignore a photoinduced redistribution of the symmetrical part $f_{v m_{s} k}^{+}=\left(f_{v m_{s} k}+f_{v \bar{m}_{s},-k}\right) / 2$.

The most important result of the microscopic theory is that both the initial and final states of the carriers involved in the optical transition contribute to the circular photogalvanic current, with different strengths and directions. The partial currents are proportional to the group velocity, being dependent on $k$, the momentum relaxation time $\tau_{p}$, and the occupation of the initial states described by the distribution function. Therefore the direction of total current depends on the details of the experimental conditions and may change its sign on varying the radiation frequency, temperature, etc. An interesting feature of the spin orientation-induced CPGE at inter-band excitation was pointed out in $[25,26]$, where it was shown that varying the frequency around the fundamental band edge results in sign inversion of the current due to the SIA contribution but not to BIA.

Inter-subband transitions: The microscopic theory of the spin orientation-induced CPGE was also developed for direct inter-subband transitions in $\mathrm{n}$-type $\mathrm{QWs}$ for both $\mathrm{C}_{\mathrm{s}}$ and $\mathrm{C}_{2 \mathrm{v}}$ symmetry; the finding was that the current is proportional to the derivative of the absorbance [24]. For the typical condition of inter-subband transitions in n-type QWs that the momentum relaxation time of photoexcited carriers in the e 2 subband, $\tau_{p}^{(2)}$, is much less than that in the e 1 subbands, $\tau_{p}^{(1)}$, it was obtained that for $\mathrm{C}_{\mathrm{s}}$ symmetry

$$
j_{x} \sim\left(\beta_{y x}^{(2)}+\beta_{y x}^{(1)}\right) \frac{\mathrm{d} \eta_{12}(\hbar \omega)}{\mathrm{d} \hbar \omega} \tau_{p}^{(1)} I P_{c i r c} \hat{e}_{y},
$$

where $\eta_{12}$ is the absorbance, $I$ is the radiation intensity, $\beta_{y x}^{(1)}$ and $\beta_{y x}^{(2)}$ are the components of $\boldsymbol{\beta}$ in the e1 and e 2 subbands, respectively. An analogous equation was obtained for QWs of $\mathrm{C}_{2 \mathrm{v}}$ symmetry. In contrast to spin-flip processes occurring for $\mathrm{C}_{\mathrm{s}}$ symmetry described above, in $\mathrm{C}_{2 \mathrm{v}}$ symmetry due to selection rules the absorption of circularly polarized radiation is spin conserving [14]. The asymmetric distribution of photoexcited electrons resulting in a current is caused by these spin-conserving but spin-dependent transitions. It was shown in [24,91] that under oblique excitation by circularly polarized light the rates of inter-subband transitions are different for electrons with the spin oriented parallel and anti-parallel to the in-plane direction of light propagation. This is depicted in figure 5(a) by vertical arrows of different thicknesses. In systems with $k$-linear spin splitting, such processes lead to an asymmetrical distribution of carriers in $k$-space, i.e. to an electrical current. Also, for this symmetry the photocurrent is proportional to the derivative of the absorption and is given by

$$
j_{x} \sim\left(\beta_{y x}^{(2)}-\beta_{y x}^{(1)}\right) \frac{\mathrm{d} \eta_{12}(\hbar \omega)}{\mathrm{d} \hbar \omega} \tau_{p}^{(1)} I P_{c i r c} \hat{e}_{y} .
$$

Since the CPGE in QW structures of $\mathrm{C}_{2 \mathrm{v}}$ symmetry is caused by spin-dependent spinconserving optical transitions, the photocurrent described by equation (23) in contrast to equation (22) is proportional to the difference of subband spin splittings. 

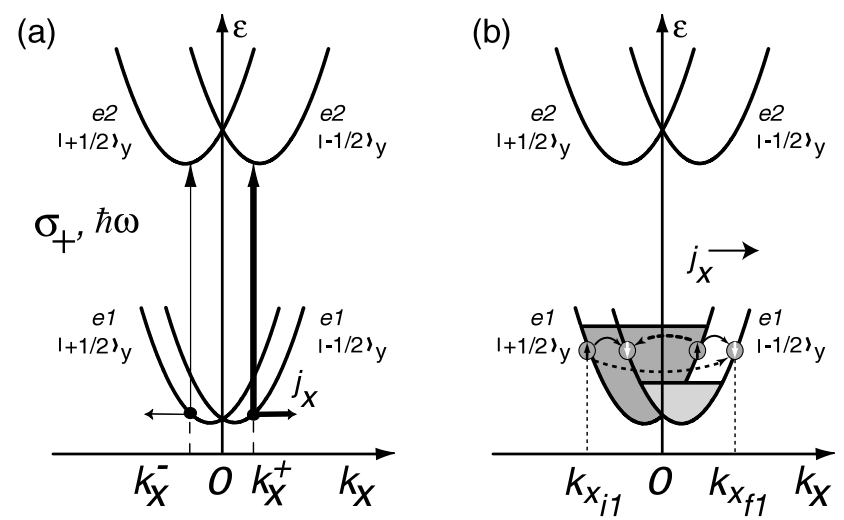

Figure 5. Microscopic pictures of the (a) CPGE and (b) spin-galvanic effect for inter-subband excitation in $\mathrm{C}_{2 \mathrm{v}}$ point group samples. In (a) the current $j_{x}$ is caused by the imbalance of optical transition probabilities at $k_{x}^{-}$and $k_{x}^{+}$decaying with the momentum relaxation time $\tau_{p}$. Excitation with $\sigma_{+}$-radiation with $\hbar \omega$ less than the energy subband separation at $k=0, \varepsilon_{21}$, induces direct spinconserving transitions (vertical arrows) at $k_{x}^{-}$and $k_{x}^{+}$. The rates of these transitions are different, as illustrated by the different thicknesses of the arrows (reversing the angle of incidence mirrors the thicknesses of the arrows). This leads to a photocurrent due to an asymmetrical distribution of carriers in $\boldsymbol{k}$-space if the splittings of the e 1 and e 2 subbands are unequal. Increasing the photon energy shifts more intense transitions to the left and less intense ones to the right, resulting in a current sign change. In (b) the current occurs after thermalization in the lowest subband which results in spin orientation in the e 1 subband. This spin-galvanic current is caused by asymmetric spin-flip scattering. The rate of spin-flip scattering depends on the value of the initial and final $\boldsymbol{k}$-vectors. Thus transitions sketched as dashed arrows yield an asymmetric occupation of the two subbands and hence a current flow which decays with the spin relaxation time $\tau_{s}$. The magnitude of the spin polarization, and hence the current, depends on the initial absorption strength but not on the momentum $k$ of the transition. Therefore the shape of the spectrum of the spin-galvanic current follows the absorption.

2.2.4. One- and two-photon excitation. One more spin photocurrent related to the photogalvanic effect was proposed in [32] and has most recently been observed in bulk GaAs [33]. The generation of the photocurrent is based on quantum interference of one- and two-photon excitation [92] which represents a coherent photogalvanic effect [34]. Irradiation of a semiconductor sample with coherent superposition of a laser beam of frequency $\omega$ satisfying $\varepsilon_{g} / 2<\hbar \omega<\varepsilon_{g}$ and its second harmonic may yield an electric current if there are definite phase relations of the radiation fields. Tuning of the phase relation among the coherent beams allows control of the current. In order to obtain a spin photocurrent, appropriate polarization of both beams is required. If the radiation fields at $\omega$ and $2 \omega$ have either the same circular polarization or orthogonal linear polarizations, quantum interference under injection distinguishes carriers of opposite spin which results in a net spin flux. It is possible to obtain spin photocurrent without electric current flow. The asymmetry of the spin population in $\boldsymbol{k}$-space appears as a consequence of spin-orbit coupling. The photocurrent is mediated by a fourth-rank tensor and thus needs no symmetry restriction as in the case of the one-photon excitation photogalvanic effect. It may be present in materials with a centre of inversion. So far, coherent control of a spin photocurrent has been observed in bulk semiconductors (GaAs) [33] but it may also be possible in QWs and in asymmetric superlattices [35].

\subsection{The spin-galvanic effect}

The picture of spin photocurrents given so far involved the asymmetry of the momentum distribution of photoexcited carriers, i.e. the spin orientation-induced CPGE. In addition to the 
CPGE a spin-driven current may also occur even after momentum relaxation of photoexcited carriers. It is due to an asymmetry of spin-flip scattering of non-equilibrium spin-polarized carriers as shown in figure 5(b). This current is caused by the spin-galvanic effect and will be described in the following.

2.3.1. Phenomenology. The spin-galvanic effect is caused by spin relaxation of a uniform non-equilibrium spin polarization in QWs of gyrotropic symmetry [29]. While this effect may occur for any method of spin orientation, e.g. electric spin injection, optical spin polarization is also possible, resulting in a spin photocurrent. Phenomenologically, an electric current can be linked to the electron's averaged spin polarization $S$ by

$$
j_{\alpha}=\sum_{\gamma} Q_{\alpha \gamma} S_{\gamma}
$$

Like in the case of $\boldsymbol{k}$-linear terms and the CPGE, here we have again a second-rank pseudotensor $\boldsymbol{Q}$ with the same symmetry restrictions as $\boldsymbol{\beta}$ and $\gamma$. Therefore in zinc-blende structure QWs, non-zero components of $Q_{\alpha \gamma}$ exist, in contrast to the case for the corresponding bulk crystals $[27,28]$. Due to tensor equivalence we have the same non-zero components of the tensor $\boldsymbol{Q}$ and their relations, as discussed above for $\boldsymbol{\beta}$ and $\boldsymbol{\gamma}$. For $\mathrm{C}_{2 \mathrm{v}}$ symmetry of (001)-grown QWs only two linearly independent components, $Q_{x y}$ and $Q_{y x}$, may be non-zero, so

$$
j_{x}=Q_{x y} S_{y}, \quad j_{y}=Q_{y x} S_{x} .
$$

Hence, a spin polarization-driven current needs a spin component lying in the plane of QWs. In $\mathrm{D}_{2 \mathrm{~d}}$ symmetry there is only one independent tensor component $Q_{x y}=Q_{y x}$. In the $\mathrm{C}_{\mathrm{s}}$ symmetry of (113)-oriented QWs an additional tensor component $Q_{x z^{\prime}}$ may be non-zero and the spin-galvanic current may be driven by spins oriented normally to the plane of the QWs.

2.3.2. The microscopic model. Microscopically, the spin-galvanic effect is caused by asymmetric spin-flip relaxation of spin-polarized electrons in systems with $\boldsymbol{k}$-linear contributions to the effective Hamiltonian [29]. Figure 6(a) sketches the electron energy spectrum along $k_{x}$ with the spin-dependent term $\beta_{y x} \sigma_{y} k_{x}$ resulting from BIA and SIA. In this case $\sigma_{y}$ is a good quantum number. Spin orientation in the $y$-direction causes unbalanced population of spin-down and spin-up subbands. The current flow is caused by $\boldsymbol{k}$-dependent spin-flip relaxation processes. Spins oriented in the $y$-direction are scattered along $k_{x}$ from the more highly filled, e.g. spin subband $|+1 / 2\rangle_{y}$, to the less filled spin subband, $|-1 / 2\rangle_{y}$. Four quantitatively different spin-flip scattering events exist and are sketched in figure 6(a) as bent arrows. The spin-flip scattering rate depends on the values of the wavevectors of the initial and the final states [47]. Therefore spin-flip transitions, shown by solid arrows in figure 6(a), have the same rates. They preserve the symmetric distribution of carriers in the subbands and, thus, do not yield a current. However, the two scattering processes shown by broken arrows are inequivalent and generate an asymmetric carrier distribution around the subband minima in both subbands. This asymmetric population results in a current flow along the $x$-direction. Within this model of elastic scattering the current is not spin polarized, since the same number of spin-up and spin-down electrons move in the same direction with the same velocity.

It must be pointed out that the above one-dimensional model, which demonstrates clearly how a spin-galvanic current can occur, somewhat simplifies the microscopic picture. The probability of the spin-flip processes $\left|+1 / 2, \boldsymbol{k}_{i}\right\rangle_{y} \rightarrow\left|-1 / 2, \boldsymbol{k}_{f}\right\rangle_{y}$ shown by arrows in figure 6 is given by the product $\left[v\left(\boldsymbol{k}_{i}-\boldsymbol{k}_{f}\right)\right]^{2}\left(\boldsymbol{k}_{f}+\boldsymbol{k}_{i}\right)^{2}$ (see equation (30) of [47]). The amplitude $v\left(\boldsymbol{k}_{f}-\boldsymbol{k}_{i}\right)$ depends on $\boldsymbol{k}_{f}-\boldsymbol{k}_{i}$ and therefore the spin-flip process is asymmetric, as needed for the occurrence of the current. However, for the one-dimensional model presented above, the probability is given by $\left[v\left(k_{x_{f}}-k_{x_{i}}\right)\right]^{2}\left(k_{x_{f}}+k_{x_{i}}\right)^{2}$. In the case of elastic scattering, as sketched 
(a)

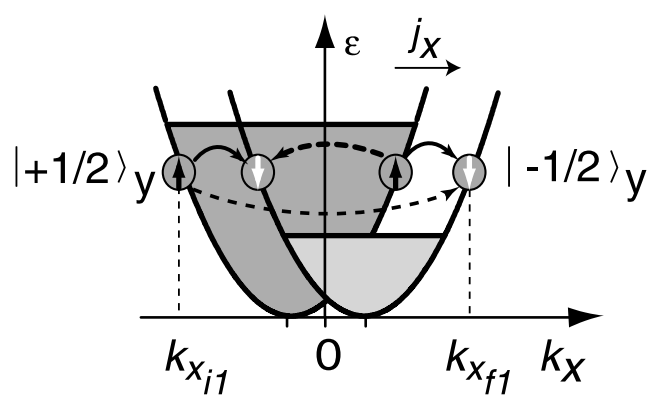

(b)

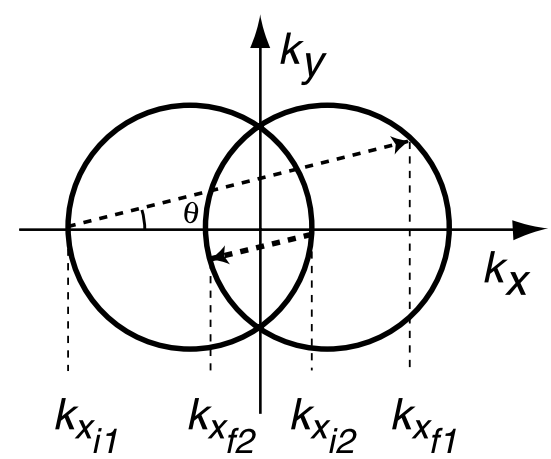

Figure 6. The microscopic origin of the spin-galvanic current in the presence of $\boldsymbol{k}$-linear terms in the electron Hamiltonian. (a) A one-dimensional sketch: the term $\sigma_{y} k_{x}$ in the Hamiltonian splits the conduction band into two parabolas with the spin $m_{s}= \pm 1 / 2$ in the $y$-direction. If one spin subband is preferentially occupied, e.g., by spin injection (the $|+1 / 2\rangle_{y}$ states in the figure), asymmetric spin-flip scattering results in a current in the $x$-direction. The rate of spin-flip scattering depends on the value of the initial and final $k$-vectors. Thus transitions sketched as dashed arrows yield an asymmetric occupation of the two subbands and hence a current flow. These transitions are also shown in two dimensions (b) by dashed arrows at the scattering angle $\theta$. If instead of the spin-down subband the spin-up subband is preferentially occupied, the current direction is reversed.

in figure 6(a), the magnitudes of the initial and final wavevectors are equal, $\left|k_{x_{i}}\right|=\left|k_{x_{f}}\right|$; thus $k_{x_{f}}+k_{x_{i}}=0$ and the probability vanishes. A non-zero current is obtained for inelastic scattering and for elastic scattering with $k_{y} \neq 0$. The latter situation is depicted in figure 6(b).

Note that the reverse of the spin-galvanic effect, i.e. a spin polarization induced by an electric current flow, has been theoretically considered in [93, 94].

The uniformity of spin polarization in space is preserved during the scattering processes. Therefore the spin-galvanic effect differs from other experimental effects where the spin current is caused by inhomogeneities. These effects will be discussed in section 6 .

2.3.3. Microscopic theory. The microscopic theory of the spin-galvanic effect has been developed in [31] for inter-subband transitions in n-type zinc-blende structure materials of $\mathrm{C}_{2 \mathrm{v}}$ symmetry. In this case the spin orientation (see figure 5(b)) is generated by resonant spin-selective optical excitation (see figure 5(a)) followed by spin-non-specific thermalization.

The occurrence of a current is due to the spin dependence of the electron scattering matrix elements $M_{k^{\prime} k}$. The $2 \times 2$ matrix $\hat{M}_{k^{\prime} k}$ can be written as a linear combination of the unit matrix $\hat{I}$ and Pauli matrices as follows:

$$
\hat{M}_{k^{\prime} k}=A_{k^{\prime} k} \hat{I}+\sigma \cdot B_{k^{\prime} k}
$$

where $A_{k^{\prime} k}^{*}=A_{k k^{\prime}}, B_{k^{\prime} k}^{*}=B_{k k^{\prime}}$ due to hermiticity of the interaction, and $A_{-k^{\prime},-k}=A_{k k^{\prime}}$, $B_{-k^{\prime},-k}=-B_{k k^{\prime}}$ due to the symmetry under time inversion. The spin-dependent part of the scattering amplitude in (001)-grown QW structures is given by [47]

$$
\boldsymbol{\sigma} \cdot \boldsymbol{B}_{\boldsymbol{k}^{\prime} \boldsymbol{k}}=v\left(\boldsymbol{k}-\boldsymbol{k}^{\prime}\right)\left[\sigma_{x}\left(k_{y}^{\prime}+k_{y}\right)-\sigma_{y}\left(k_{x}^{\prime}+k_{x}\right)\right] .
$$

We note that equation (27) determines the spin relaxation time, $\tau_{s}^{\prime}$, due to the Elliot-Yafet 
mechanism. The spin-galvanic current, for instance in the $y$-direction, has the form [31]

$j_{S G E, x}=Q_{x y} S_{y} \sim e n_{e} \frac{\beta_{y x}^{(1)}}{\hbar} \frac{\tau_{p}}{\tau_{s}^{\prime}} S_{y}, \quad j_{S G E, y}=Q_{y x} S_{x} \sim e n_{e} \frac{\beta_{x y}^{(1)}}{\hbar} \frac{\tau_{p}}{\tau_{s}^{\prime}} S_{x}$.

Since scattering is the origin of the spin-galvanic effect, the spin-galvanic current, $j_{S G E}$, is determined by the Elliot-Yafet spin relaxation time. The relaxation time $\tau_{s}^{\prime}$ is proportional to the momentum relaxation time $\tau_{p}$. Therefore the ratio $\tau_{p} / \tau_{s}^{\prime}$ in equations (28) does not depend on the momentum relaxation time. The in-plane average spin $S_{x}$ in equations (28) decays with the total spin relaxation time $\tau_{s}$ (which may have a contribution from any spin relaxing process). Thus the time decay of the spin-galvanic current following a pulsed photoexcitation is determined by $\tau_{s}$.

For the case where spin relaxation is obtained as a result of inter-subband absorption of circularly polarized radiation, the current is given by

$$
\begin{aligned}
& j_{S G E, x}=Q_{x y} S_{y} \sim e \frac{\beta_{y x}^{(1)}}{\hbar} \frac{\tau_{p} \tau_{s}}{\tau_{s}^{\prime}} \frac{\eta_{12} I}{\hbar \omega} P_{c i r c} \xi \hat{e}_{y}, \\
& j_{S G E, y}=Q_{y x} S_{x} \sim e \frac{\beta_{x y}^{(1)}}{\hbar} \frac{\tau_{p} \tau_{s}}{\tau_{s}^{\prime}} \frac{\eta_{12} I}{\hbar \omega} P_{c i r c} \xi \hat{e}_{x} .
\end{aligned}
$$

where $\eta_{12}$ is the absorbance in the transitions between e 1 and e 2 subbands. The parameter $\xi$, varying between 0 and 1, is the ratio of photoexcited electrons relaxing to the e 1 subband with and without spin flips. It determines the degree of spin polarization in the lowest subband (see figure 4(b)) and depends on the details of the relaxation mechanism. Optical orientation requires $\xi \neq 0[3,91,95]$. Equations (29) show that the spin-galvanic current is proportional to the absorbance and is determined by the spin splitting in the first subband, $\beta_{y x}^{(1)}$ or $\beta_{x y}^{(1)}$.

2.3.4. The spin-galvanic effect for optical orientation. Excitation of QWs by circularly polarized light results in a spin polarization which, at the appropriate orientation of the electron spins, causes a photocurrent due to the spin-galvanic effect. Because of the tensor equivalence, the spin-galvanic current induced by circularly polarized light always occurs simultaneously with the spin orientation-induced CPGE. The two effects can be separated by time-resolved measurements because of the different relaxation mechanisms of the two currents. After removal of light or under pulsed photoexcitation, the circular photogalvanic current decays with the momentum relaxation time whereas the spin-galvanic current decays with the spin relaxation time. On the other hand, as has been recently shown [31], in inter-subband transitions the spin-galvanic effect may be separated from the CPGE making use of the spectral behaviour at resonance. The optically induced spin-galvanic current reproduces the absorbance, whereas the CPGE is proportional to the derivative of the absorbance and vanishes at the resonance frequency. This will be discussed in more detail in section 4.2.2.

2.3.5. The spin-galvanic effect for optical orientation in the presence of a magnetic field. Another possibility for investigating the spin-galvanic effect without contributions of the spin orientation-induced CPGE to the current was introduced in [29]. This method is not limited to resonant inter-subband optical excitation. The spin polarization was obtained by absorption of circularly polarized radiation at normal incidence on (001)-grown QWs as depicted in figure 7. For normal incidence the spin orientation-induced CPGE as well as the spin-galvanic effect vanish because $\hat{e}_{x}=\hat{e}_{y}=0$ (see equations (14)) and $S_{x}=S_{y}=0$ (see equations (25)), respectively. Thus, we obtain a spin orientation along the $z$-coordinate but no spin photocurrent.

The steady-state spin polarization $S_{0 z}$ is proportional to the spin generation rate $\dot{S}_{z}$. To obtain an in-plane component of the spins, necessary for the spin-galvanic effect, a magnetic 


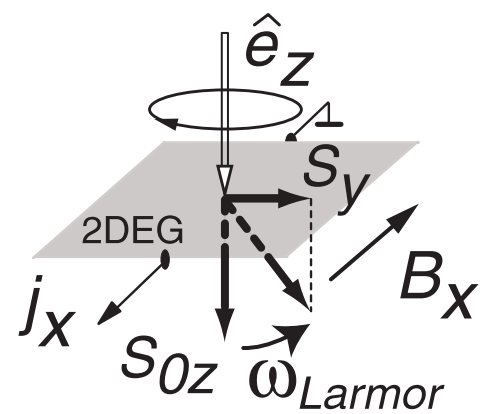

Figure 7. The optical scheme for generating a uniform in-plane spin polarization which causes spin-galvanic photocurrent. Electron spins are oriented normal to the plane of the QW sy circularly polarized radiation and rotated into the plane by Larmor precession in a magnetic field $B_{x}$.

field $\boldsymbol{B} \| x$-axis has been applied. Due to Larmor precession, a non-equilibrium spin polarization $S_{y}$ is induced:

$$
S_{y}=-\frac{\omega_{L} \tau_{s \perp}}{1+\left(\omega_{L} \tau_{s}\right)^{2}} S_{0 z},
$$

where $\tau_{s}=\sqrt{\tau_{s \|} \tau_{s \perp}}, \tau_{s \|}, \tau_{s \perp}$ are the longitudinal and transverse electron spin relaxation times, $\omega_{L}$ is the Larmor frequency. The denominator in equation (30) yielding the decay of $S_{y}$ for $\omega_{L}$ exceeding the inverse spin relaxation time is well known from the Hanle effect $[3,96]$

On a phenomenological level and at small magnetic fields, $\omega_{L} \tau_{s} \ll 1$, this magnetic field-induced spin photocurrent can be described by

$$
j_{\alpha}=\sum_{\beta \gamma} \mu_{\alpha \beta \gamma} B_{\beta} \mathrm{i}\left(\boldsymbol{E} \times \boldsymbol{E}^{*}\right)_{\gamma}=\sum_{\beta \gamma} \mu_{\alpha \beta \gamma} B_{\beta} \hat{\boldsymbol{e}}_{\gamma} E_{0}^{2} P_{c i r c}
$$

where $\mu_{\alpha \beta \gamma}$ is a third-rank tensor. As $P_{\text {circ }}$ is a pseudo-scalar and $\boldsymbol{B}$ a pseudo-vector, $\mu_{\alpha \beta \gamma}$ is an ordinary negative-parity third-rank tensor which is allowed in inversion asymmetric materials only. Gyrotropy at zero magnetic field, as in the case of only an optical excited spin-galvanic effect or the CPGE, is not necessary. We note that in non-gyrotropic p-type bulk GaAs a magnetic field-induced CPGE was previously observed upon intra-band excitation [97]. However, this effect is not due to spin orientation and does not occur in p-type QWs due to spatial quantization [98]. In QWs under normal incidence of the light and for a magnetic field lying in the plane of a $\mathrm{QW}$ of $\mathrm{C}_{2 \mathrm{v}}$ symmetry, which corresponds to the measurements in section 4.2.1, the current is described by two independent components of the tensor $\mu$ and can be written as

$$
j_{x}=\mu_{x x z} B_{x} \hat{e}_{z} E_{0}^{2} P_{\text {circ }}, \quad j_{y}=\mu_{y y z} B_{y} \hat{e}_{z} E_{0}^{2} P_{\text {circ }} .
$$

The current $\boldsymbol{j}$ and the magnetic field $\boldsymbol{B}$ are parallel (or anti-parallel) when the magnetic field is applied along $\langle 110\rangle$ and neither parallel nor perpendicular for $B \|\langle 100\rangle$. In $\mathrm{D}_{2 \mathrm{~d}}$ symmetry QWs with symmetric interfaces, $\mu_{x x z}=-\mu_{y y z}$, and therefore the current is perpendicular to the magnetic field for $B \|\langle 100\rangle$.

\subsection{The spin orientation-induced circular photogalvanic effect versus the spin-galvanic effect}

The spin orientation-induced CPGE and the spin-galvanic effect have in common that the current flow is driven by an asymmetric distribution of carriers in $\boldsymbol{k}$-space in systems with lifted spin degeneracy due to $k$-linear terms in the Hamiltonian. The crucial difference between the 
(a)

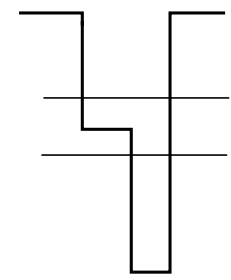

(b)

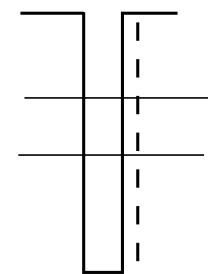

(c)

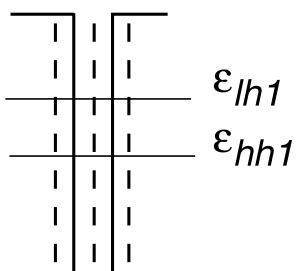

Figure 8. Potential profiles of SiGe QW samples: (a) a compositionally stepped QW, (b) an asymmetrically doped compositionally symmetric QW, and (c) a symmetric QW. The vertical dashed lines indicate the doping.

two effects is that the spin-galvanic effect may be caused by any kind of spin injection, while the spin orientation-induced CPGE needs optical excitation with circularly polarized radiation. Even if the spin-galvanic effect is achieved by optical spin orientation, as discussed here, the microscopic mechanisms are different. The spin-galvanic effect is caused by asymmetric spinflip scattering of spin-polarized carriers and it is determined by the process of spin relaxation (see figure 6). If spin relaxation is absent, the spin-galvanic current vanishes. In contrast, the spin orientation-induced CPGE is the result of selective photoexcitation of carriers in $k$-space with circularly polarized light due to optical selection rules and depends on the momentum relaxation (see figure 2). In some optical experiments the observed photocurrent may represent a sum of the two effects. For example, if we irradiate an (001)-oriented QW with obliquely incident circularly polarized radiation, we obtain both selective photoexcitation of carriers in $\boldsymbol{k}$-space determined by momentum relaxation and a spin-galvanic effect due to an in-plane component of non-equilibrium spin polarization. Thus both effects contribute to the current occurring in the plane of the QWs. The two mechanisms can be distinguished by time-resolved measurements.

\section{Methods}

\subsection{Samples}

The experiments were carried out on GaAs, InAs, semimagnetic BeZnMnSe, and SiGe heterostructures belonging to two different classes of symmetry. The more highly symmetric structures were (001)-oriented QWs. While these structures can belong to two point groups, either $\mathrm{D}_{2 \mathrm{~d}}$ or $\mathrm{C}_{2 \mathrm{v}}$, our measurements showed that all samples available for the present work correspond to the point group $C_{2 v}$. The structures of the lower-symmetry class $C_{\mathrm{s}}$ were (113)oriented QWs and QWs grown on (001) miscut substrates.

Zinc-blende structure-based QW samples were of n- and p-type GaAs/AlGaAs grown by molecular beam epitaxy (MBE) with QW widths $L_{W}$ of 4-20 nm [20, 24, 38], n-type InAs/AlGaSb QWs with $L_{W}=15 \mathrm{~nm}[20,99]$, as well as single n-type GaAs heterojunctions. The free carrier densities, $n_{s}$ for electrons and $p_{s}$ for holes, ranged from $10^{11}$ to $2 \times 10^{12} \mathrm{~cm}^{-2}$. The mobility at $4.2 \mathrm{~K}$ in n-type samples was from $5 \times 10^{5}$ to $2 \times 10^{6} \mathrm{~cm}^{2} \mathrm{~V}^{-1} \mathrm{~s}^{-1}$ and in p-type samples was about $5 \times 10^{5} \mathrm{~cm}^{2} \mathrm{~V}^{-1} \mathrm{~s}^{-1}$.

Semimagnetic BeZnMnSe semiconductor heterostructures were grown by MBE on semiinsulating GaAs substrates with (001) orientation [43, 100]. The heterostructures consisted of a $500 \mathrm{~nm}$ thick $\mathrm{Be}_{0.03} \mathrm{Zn}_{0.97} \mathrm{Se}$ layer, $\mathrm{n}$ doped to $2 \times 10^{18} \mathrm{~cm}^{-3}$, followed by a $100 \mathrm{~nm}$ thick $\mathrm{Be}_{0.05} \mathrm{Zn}_{0.89} \mathrm{Mn}_{0.06}$ Se layer, $\mathrm{n}$ doped to $6 \times 10^{18} \mathrm{~cm}^{-3}$. 

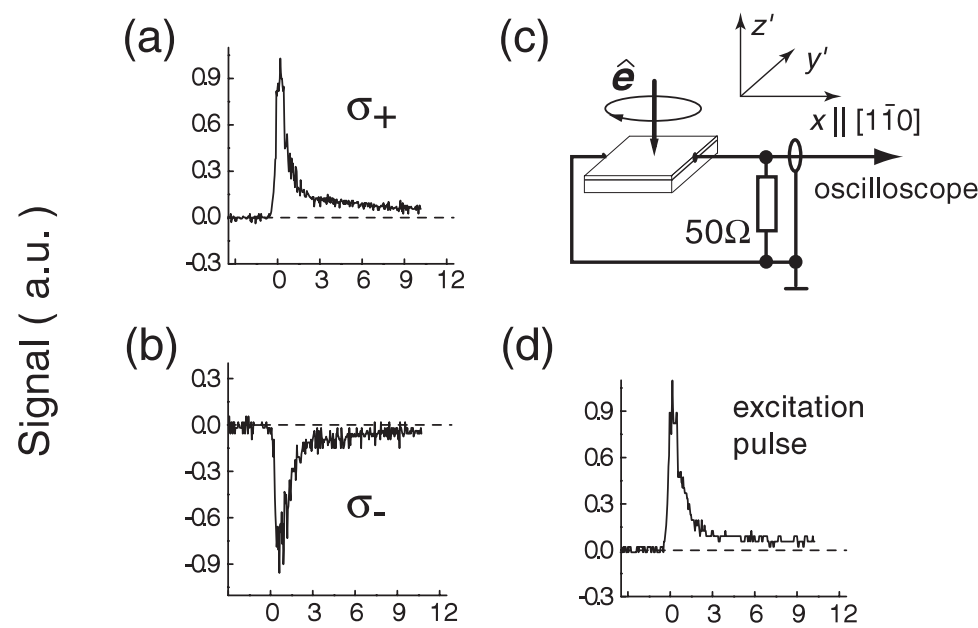

(d)

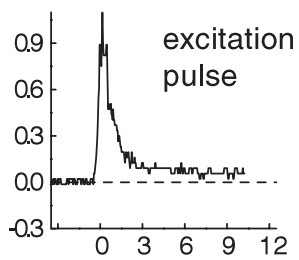

Time $\left(10^{-7} \mathrm{~s}\right)$

Figure 9. Oscilloscope traces obtained for pulsed excitation of (113)-grown n-type GaAs QWs at $\lambda=10.6 \mu \mathrm{m}$. (a) and (b) show CPGE signals obtained for circular $\sigma_{+}$- and $\sigma_{-}$-polarization, respectively. For comparison, in (d) a signal pulse for a fast-photon drag detector is plotted. In (c) the measurement arrangement is sketched. For (113)-grown samples of $\mathrm{C}_{\mathrm{s}}$ symmetry, radiation was applied at normal incidence and the current detected in the direction $x \|$ [110]. For (001)-grown QWs, oblique incidence was used in order to produce a helicity-dependent current.

The measurements on SiGe QWs were carried out on p-type structures, MBE grown on (001)- and (113)-oriented Si substrates [23]. Both bulk Si and Ge have a centre of inversion; therefore in order to obtain gyrotropy, asymmetric QWs were grown. Two groups of (001)grown asymmetric samples, whose potential profiles are sketched in figures 8(a) and (b), were fabricated in the following manner: one of the groups of samples was compositionally stepped (figure 8(a)), comprising ten QWs of $\left[\mathrm{Si}_{0.75} \mathrm{Ge}_{0.25}(4 \mathrm{~nm}) / \mathrm{Si}_{0.55} \mathrm{Ge}_{0.45}(2.4 \mathrm{~nm})\right]$ separated by $6 \mathrm{~nm}$ Si barriers. The second group of asymmetric structures had a single QW of $\mathrm{Si}_{0.75} \mathrm{Ge}_{0.25}$ composition which was doped with boron from one side only (figure 8(b)). These structures are of the $C_{2 v}$ point group symmetry, as was confirmed by the experiments described below. Structures with the lower symmetry $\mathrm{C}_{\mathrm{s}}$ were (113) grown with a $\mathrm{Si} / \mathrm{Si}_{0.75} \mathrm{Ge}_{0.25}(5 \mathrm{~nm}) / \mathrm{Si}$ single QW, one-side boron doped. As a reference sample, a (001)-grown compositionally symmetric and symmetrically boron-doped multiple-QW structure (figure 8(c)) of sixty $\mathrm{Si}_{0.7} \mathrm{Ge}_{0.3}(3 \mathrm{~nm}$ ) QWs has been used. All these samples had free carrier densities $p_{s}$ of about $8 \times 10^{11} \mathrm{~cm}^{-2}$ in each QW.

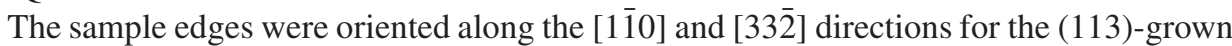
sample and along the [110] and [110] directions for the (001)-grown one. For (113)-oriented samples, two pairs of ohmic contacts were centred along opposite sample edges, pointing in the directions $x \|[1 \overline{10}]$ and $y \|[33 \overline{2}]$ (see figure 9 and the inset in figure 10, lower plate). For (001)-oriented samples, two pairs of point contacts in the middle of the sample edges with connecting lines along $x \|[1 \overline{10}]$ and $y^{\prime} \|[110]$ were prepared (see the inset in figure 10, upper plate). These samples had two additional pairs of contacts at the corners of the samples corresponding to the $\langle 100\rangle$ directions (see the inset in figure 10, upper plate). 


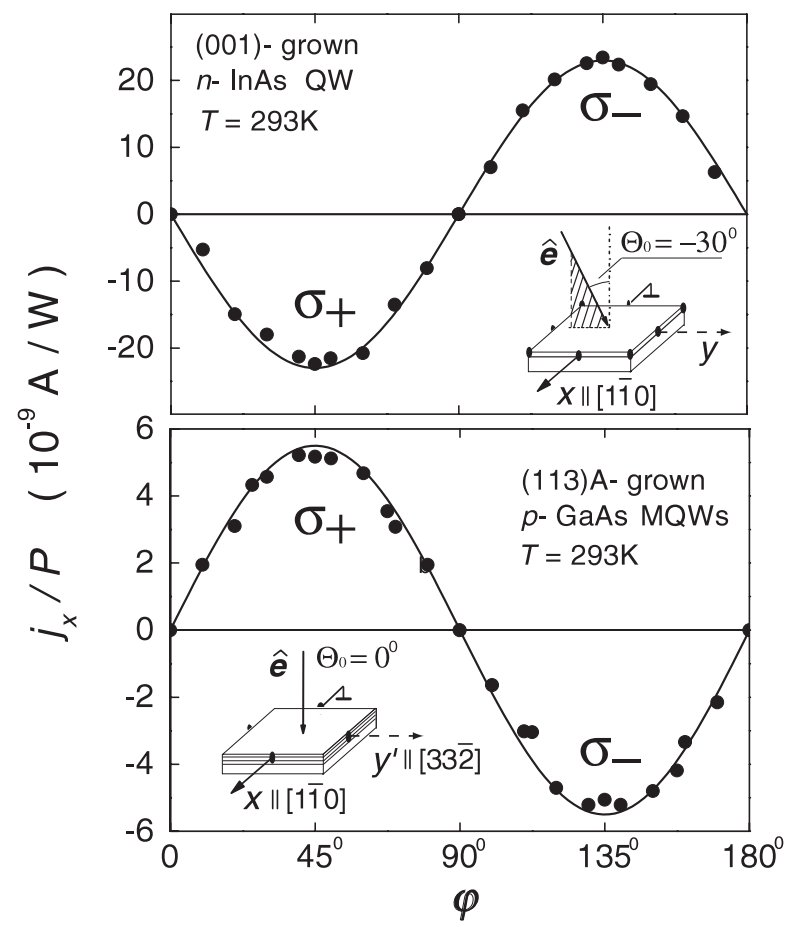

Figure 10. The photocurrent in QWs normalized by the light power $P$ as a function of the phase angle $\varphi$ defining helicity. Measurements are presented for $T=293 \mathrm{~K}$ and $\lambda=76 \mu \mathrm{m}$. The insets show the geometry of the experiment. Upper panel: oblique incidence of radiation with an angle of incidence $\Theta_{0}=-30^{\circ}$ on n-type (001)-grown InAs/AlGaSb QWs (symmetry class $\mathrm{C}_{2 \mathrm{v}}$ ). The current $j_{x}$ is perpendicular to the direction of light propagation. Lower panel: normal incidence of radiation on p-type (113)A-grown GaAs/AlGaAs QWs (symmetry class $\mathrm{C}_{\mathrm{s}}$ ). The current $j_{x}$ flows along the [110] direction perpendicular to the mirror plane of $\mathrm{C}_{\mathrm{s}}$ symmetry. Full curves show an ordinate scale fit using equations (14) and (15) for the top and lower panel, respectively.

\subsection{The experimental technique}

For optical excitation, mid-infrared (MIR), far-infrared (FIR), and visible laser radiations were used. Most of the measurements were carried out in the infrared with photon energies less than the energy gap of the semiconductors investigated. For investigations of spin photocurrents, infrared excitation has several advantages. First of all, below the energy gap the absorption is very weak and therefore allows homogeneous excitation with marginal heating of the 2D electron gas. Furthermore, in contrast to the case for inter-band excitation, there are no spurious photocurrents due to other mechanisms such as the Dember effect, photovoltaic effects at contacts, and Schottky barriers. Depending on the photon energy and QW band structure, the MIR and FIR radiation induce direct optical transitions between size-quantized subbands in $\mathrm{n}$ - and p-type samples or, at longer wavelength, indirect optical transitions (Drude absorption) in the lowest subband.

A high-power pulsed MIR transversely excited atmospheric pressure carbon dioxide $\left(\mathrm{TEA}-\mathrm{CO}_{2}\right)$ laser and a molecular FIR laser [101, 102] were used as radiation sources in the spectral range between 9.2 and $496 \mu \mathrm{m}$. The corresponding photon energies $\hbar \omega$ lie in the range of $135-2 \mathrm{meV}$. The radiation pulses $(\simeq 100 \mathrm{~ns})$ of power $P$ up to $50 \mathrm{~kW}$ were focused 
to a spot of about $1 \mathrm{~mm}^{2}$ yielding a maximum intensity of about $5 \mathrm{MW} \mathrm{cm}^{-2}$. Such high intensities are only needed for the saturation measurements described in section 4.4. The power required to detect spin photocurrents is much lower. One series of measurements was carried out making use of the frequency tunability of the free electron laser FELIX at FOM-Rijnhuizen in The Netherlands [103]. The FELIX operated in the spectral range between 7 and $12 \mu \mathrm{m}$. The output pulses of light from FELIX were chosen to be 3 ps long, separated by $40 \mathrm{~ns}$, in a train (or 'macropulse') of $5 \mu$ s duration. The macropulses had a repetition rate of $5 \mathrm{~Hz}$.

Typically, this laser emits linearly polarized radiation. The polarization was modified from linear to circular using a Fresnel rhomb and $\lambda / 4$ plates for MIR and FIR radiation, respectively. The helicity $P_{\text {circ }}$ of the incident light was varied from -1 (left-handed circular, $\sigma_{-}$) to +1 (right-handed circular, $\sigma_{+}$) according to $P_{\text {circ }}=\sin 2 \varphi$ (see equation (12)). In the present experimental arrangement, the phase angle $\varphi$ corresponds to the angle between the initial plane of polarization and the optical axis of the $\lambda / 4$ plate or the polarization plane of the Fresnel rhomb.

For optical inter-band excitation, a cw Ti:sapphire laser was used, providing radiation of $\lambda=0.777 \mu \mathrm{m}$ with about $100 \mathrm{~mW}$ power. In order to extract the helicity-dependent current the linearly polarized laser beam was transmitted through a photoelastic modulator which yields a polarization oscillating periodically between $\sigma_{+}$and $\sigma_{-}$[29].

Samples were studied at room temperature or mounted in an optical cryostat which allowed the variation of the temperature in the range of 4.2-293 K. The photocurrent $j_{x}$ was measured in the unbiased structures via the voltage drop across a $50 \Omega$ load resistor in a closed-circuit configuration [10] (see figure 9(c)). The current in the case of the excitation by visible radiation was recorded by a lock-in amplifier in phase with the photoelastic modulator.

The experiments on the spin-galvanic effect, which require an external magnetic field, were performed at room temperature in a conventional electromagnet with the magnetic field up to $1 \mathrm{~T}$ and at $4.2 \mathrm{~K}$ using a superconducting split-coil magnet with $B$ up to $3 \mathrm{~T}$.

\section{Experimental results and discussion}

\subsection{The spin polarization-induced circular photogalvanic effect}

4.1.1. General features. With illumination of QW structures by polarized radiation a current signal proportional to the helicity $P_{\text {circ }}$ has been observed in unbiased samples [10, 20, 23, 43]. The irradiated QW structure represents a current source wherein the current flows in the QW. Figure 9 shows measurements of the voltage drop across a $50 \Omega$ load resistor in response to $100 \mathrm{~ns}$ laser pulses at $\lambda=10.6 \mu \mathrm{m}$. Signal traces are plotted in figure 9(a) for right-handed circular polarization and in figure 9 (b) for left-handed circular polarization, in comparison to a reference signal (see figure 9(d)) obtained from the fast-photon drag detector [104, 105]. The signal follows the temporal structure of the applied laser pulses. In figure 10 the current is shown as a function of the phase angle $\varphi$. The current signal assumes a maximum for circularly polarized radiation and changes sign if the polarization is switched from $\sigma_{+}$to $\sigma_{-}$. In the case of linearly polarized radiation corresponding to $\varphi=0^{\circ}$ or $90^{\circ}$ the current vanishes. The radiation-induced current and its characteristic helicity dependence reveal that we are dealing with a CPGE. The effect is quite general and has been observed in all samples in the temperature range of 4.2-293 $\mathrm{K}$ and over a wide spectral range.

In (001)-oriented samples a helicity-dependent signal is only observed under oblique incidence [20, 21, 23]. For light propagating along the $\langle 110\rangle$ direction the photocurrent flows perpendicular to the wavevector of the incident light (see figure 10, upper plate). This observation is in accordance with equations (14). For illumination along a cubic axis 
$\langle 100\rangle$, both a transverse and longitudinal circular photogalvanic current are detected [22]. The presence of a transverse current in this geometric configuration in all (001)-oriented samples, except miscut ones, investigated so far unambiguously demonstrates that they belong to the symmetry class $C_{2 \mathrm{v}}$. Indeed, in such a geometry $\left(\hat{e}_{x}=\hat{e}_{y}=1 / \sqrt{2}\right)$, the transverse effect is only allowed for the $\mathrm{C}_{2 \mathrm{v}}$ symmetry class and is forbidden for $\mathrm{D}_{2 \mathrm{~d}}$ symmetry as can be seen from equations (13) and (14) and the discussion following. In samples grown on a (113) GaAs surface or on (001) miscut substrates representing the lower-symmetry class $\mathrm{C}_{\mathrm{s}}$, the CPGE has been observed also under normal incidence of radiation [10, 20, 21, 23] as shown in the lower plate of figure 10. This is in contrast to the case for (001)-oriented samples and in accordance with the phenomenological theory of the CPGE for $C_{s}$ (see equations (15)). For normal incidence in this symmetry, the current always flows along the [110] direction perpendicular to the plane of mirror reflection of the point group $\mathrm{C}_{\mathrm{s}}$. The solid curves in figure 10 are obtained from the phenomenological picture outlined above which perfectly describes the experimental observations.

In figure 11 we show the dependence of the photocurrent on the angle of incidence $\Theta_{0}$ of the right-handed circularly polarized laser beam. For (001)-oriented samples $\left(\mathrm{C}_{2 \mathrm{v}}\right.$ symmetry) a variation of $\Theta_{0}$ in the plane of incidence normal to $x$ changes the sign of the current $j_{x}$ for normal incidence, $\Theta_{0}=0$, as can be seen in the upper panel of figure 11. The lower panel of figure 11 displays the angular dependence for (113)-oriented QWs $\left(\mathrm{C}_{\mathrm{s}}\right.$ symmetry). The currents measured as a function of the angle of incidence $\Theta_{0}$ along any direction in the plane of (001)-oriented samples and along $x \|$ [1 10 ] for (113)-oriented samples (figure 11) are in very good agreement with the phenomenological expressions (14), (16), and (18) for $\mathrm{C}_{2 \mathrm{v}}$ and equations (15), (17), and (18) for $\mathrm{C}_{\mathrm{s}}$ symmetry. Both figures show experimental data compared to calculations which were fitted with one ordinate scaling parameter. The fact that $j_{x}$ is an even function of $\Theta_{0}$ for (113)-oriented samples means that in the sample under study the component $\gamma_{x z^{\prime}}$ of $\gamma$ in equations (15) is much larger than $\gamma_{x y^{\prime}}$.

Microscopically, the CPGE can be the result of different optical absorption mechanisms such as inter-band transitions, inter-subband transitions in QWs, and Drude absorption. The CPGE for inter-band absorption (valence band to conduction band) has not been observed experimentally so far. A strong spurious photocurrent due to other mechanisms such as the Dember effect and photovoltaic effects at contacts mask the relatively weak CPGE. However, application of polarization-selective measurements, such as modulation of the polarization, should allow us to extract the CPGE current. In the infrared range, where the effects mentioned above vanish, the CPGE has been observed experimentally. In QW structures, absorption of infrared radiation may occur through indirect intra-subband optical transitions (Drude absorption) and, for photon energies in resonance with the energy distance between sizequantized subbands, through direct transitions between these subbands.

4.1.2. Inter-subband transitions in n-type $Q W s$. Absorption of radiation in the range of 9 to $11 \mu \mathrm{m}$ in n-type GaAs samples of QW widths 8.2 and $8.6 \mathrm{~nm}$ is dominated by resonant direct inter-subband optical transitions between the first and the second size-quantized subbands. Figure 12 shows the resonance behaviour of the absorption measured in GaAs QWs obtained by Fourier spectroscopy using a multiple-reflection waveguide geometry. Applying MIR radiation from the $\mathrm{CO}_{2}$ laser, which causes direct transitions in GaAs QWs, a current signal proportional to the helicity $P_{\text {circ }}$ has been observed at normal incidence in (113)-oriented samples and at oblique incidence in (001)-oriented samples, indicating a spin orientationinduced CPGE [24]. In figure 12 the data are presented for a (001)-grown n-type GaAs QW of $8.2 \mathrm{~nm}$ width measured at room temperature. It is seen that the current for both left- and right-handed circular polarization changes sign at a frequency $\omega=\omega_{i n v}$. This inversion 


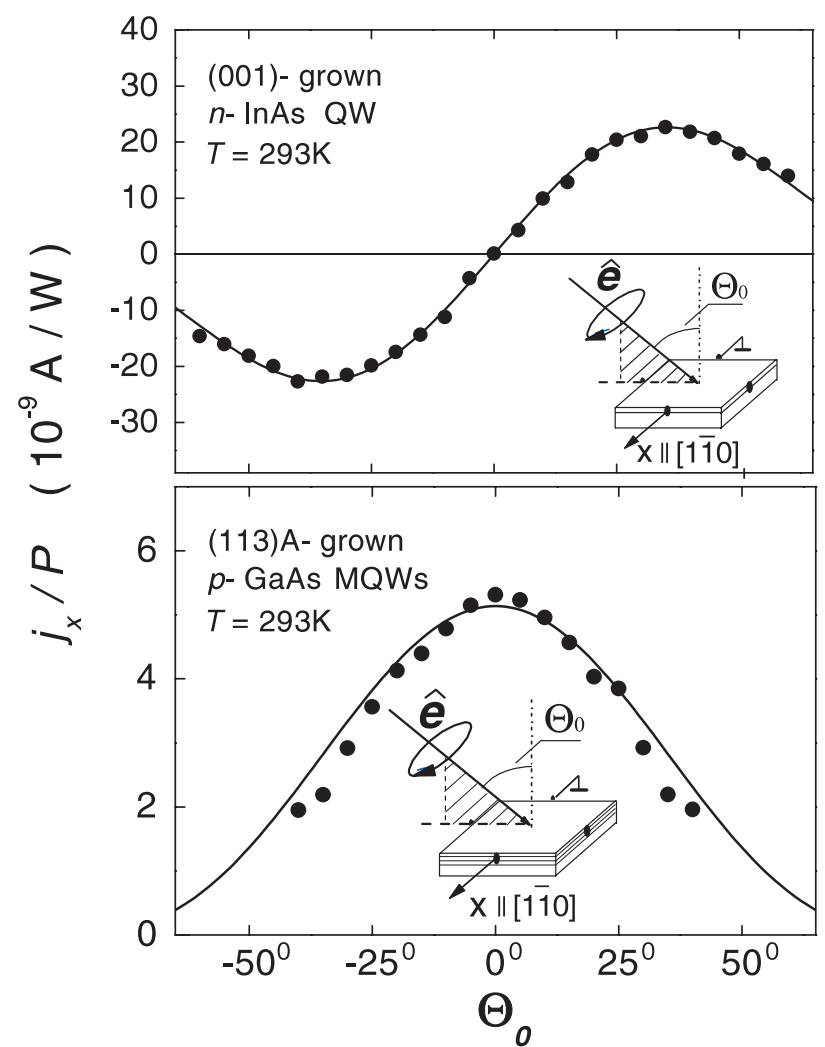

Figure 11. The photocurrent in QWs normalized by $P$ as a function of the angle of incidence $\Theta_{0}$ for right-handed circularly polarized radiation $\sigma_{+}$measured perpendicular to the light propagation direction $(T=293 \mathrm{~K}, \lambda=76 \mu \mathrm{m})$. Upper panel: n-type (001)-grown InAs/AlGaSb QWs $\left(\mathrm{C}_{2 \mathrm{v}}\right)$. Lower panel: p-type (113)A-grown GaAs/AlGaAs QWs $\left(\mathrm{C}_{\mathrm{s}}\right)$. Full curves show an ordinate scale fit using equations (14), (16) (upper plate) and (15), (17) (lower plate).

frequency $\omega_{i n v}$ coincides with the frequency of the absorption peak. The peak frequency and $\omega_{i n v}$ depends on the sample width in agreement with the variation of the subband energy separation. Experimental results shown in figure 12, in particular the sign inversion of the spectral behaviour of the current, are in good agreement with microscopic theory developed in [24] (see equations (22) and (23)). The spectral sign inversion of the CPGE has also been detected in a (113)-oriented n-type GaAs QW which belongs to the point group $\mathrm{C}_{\mathrm{s}}$. In this case the helicity-dependent signal is observed in the $x$-direction for normal incidence of radiation along $z^{\prime}$.

The inversion of the photon helicity-driven current is a direct consequence of $\boldsymbol{k}$-linear terms in the band structure of subbands together with energy and momentum conservation, as well as optical selection rules for direct optical transitions between size-quantized subbands [24]. At large photon energy, $\hbar \omega>\varepsilon_{21}$, and for QWs of $\mathrm{C}_{\mathrm{s}}$ symmetry, excitation occurs at positive $k_{x}$ resulting in a current $j_{x}$ shown by an arrow in figure 2(b). Decreasing the photon frequency shifts the transition towards negative $k_{x}$ and reverses the direction of the current. In the framework of this model the inversion of sign of the current takes place at the photon energy $\hbar \omega_{i n v}$ corresponding to optical transitions from the band minima. This shift of $\omega_{i n v}$ away from the frequency of peak absorption cannot be resolved in experiments on currently available 


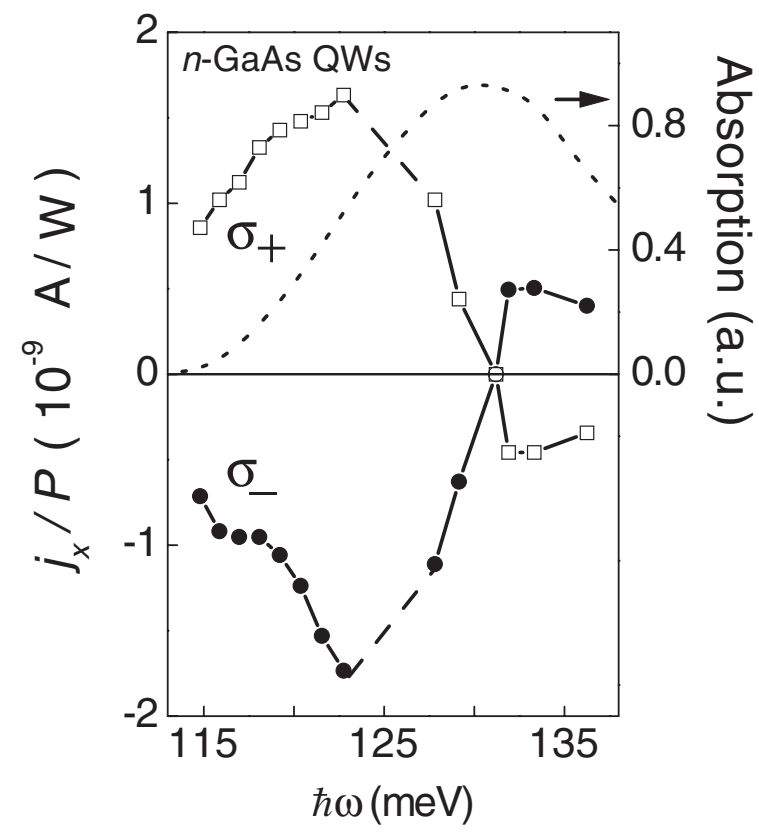

Figure 12. The photocurrent in QWs normalized by $P$ as a function of the photon energy $\hbar \omega$. Measurements are presented for n-type (001)-grown GaAs/AlGaAs QWs of $8.2 \mathrm{~nm}$ width (symmetry class $\mathrm{C}_{2 \mathrm{v}}$ ) at $T=293 \mathrm{~K}$ and oblique incidence of radiation with an angle of incidence $\Theta_{0}=20^{\circ}$. The absorption of the MIR laser radiation results in direct transitions between e1 and e2 subbands. The current $j_{x}$ is perpendicular to the direction of the light propagation. The dotted curve shows the absorption measured using a Fourier spectrometer.

samples because of the broadening of the absorption [24]. Similar arguments hold for $\mathrm{C}_{2 \mathrm{v}}$ symmetry (relevant for (001)-oriented samples) for oblique incidence (see figure 5(a)), although the simple selection rules are no longer valid $[14,106]$. By the selection rules, the absorption of circularly polarized radiation is spin conserving but the rates of inter-subband transitions are different for electrons with the spin oriented parallel and anti-parallel to the in-plane direction of light propagation [91]. The asymmetric distribution of photoexcited electrons results in a current which is caused by these spin-conserving but spin-dependent transitions [24].

4.1.3. Inter-subband transitions in p-type $Q W s$. The helicity-dependent current of the spin orientation-induced CPGE has also been observed in p-type GaAs QWs due to transitions between heavy-hole (hh1) and light-hole (lh1) subbands demonstrating spin orientation of holes (see figure 10, lower plate) $[10,20,38]$. QWs with various widths in the range of 4$20 \mathrm{~nm}$ were investigated. For direct inter-subband transitions, photon energies between 35 and $8 \mathrm{meV}$ of FIR radiation corresponding to these QW widths were applied. Due to the different effective masses of the light and heavy holes the absorption does not show narrow resonances. Cooling the sample from room temperature to $4.2 \mathrm{~K}$ leads to a change of sign of the spin orientation-induced CPGE but the $\sin 2 \varphi$ dependence is retained [10]. This temperaturedependent change of sign of the photogalvanic current, which was also observed in n-type samples for direct transitions, may be caused by the change of scattering mechanism from impurity scattering to phonon-assisted scattering (see section 2.2.3). 


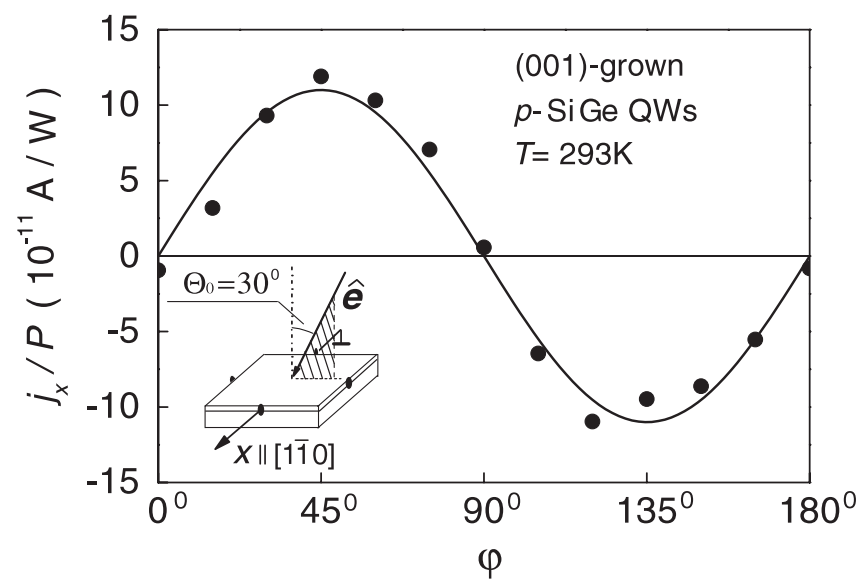

Figure 13. The photogalvanic current $j_{x}$ normalized by $P$ in (001)-grown and asymmetrically doped $\mathrm{SiGe}$ QWs and measured at room temperature as a function of the phase angle $\varphi$. The data were obtained for oblique incidence of irradiation at $\lambda=10.6 \mu \mathrm{m}$. The full line was fitted using equations (14). The inset shows the geometry of the experiment.

4.1.4. Intra-subband transitions in QWs. Optical absorption caused by indirect transitions in n-type samples has been obtained by applying FIR radiation covering the range of 76-280 $\mu \mathrm{m}$ corresponding to photon energies from 16 to $4.4 \mathrm{meV}$. The experiments were carried out on GaAs [20, 21], InAs [20], and semimagnetic ZnBeMnSe [43] QWs. The energy separation between the e1 and e2 size-quantized subbands of those samples is much larger than the FIR photon energies used here. Therefore the absorption is caused by indirect intra-subband optical transitions. For illumination of (001)-grown QWs with obliquely incident FIR radiation a current signal proportional to the helicity $P_{\text {circ }}$ has been observed (see figure 10, upper plate), showing that Drude absorption of a $2 \mathrm{D}$ electron gas results in spin orientation and a CPGE. A spin orientation-induced CPGE occurring upon intra-subband absorption was also observed in p-type samples at long wavelengths [10, 21, 23], where the photon energies are smaller than the energy separation between the first heavy-hole and the first light-hole subbands.

4.1.5. The spin orientation-induced CPGE in SiGe QWs. In symmetrically (001)-grown and symmetrically doped SiGe QWs no photogalvanic current has been observed, as expected from the presence of inversion symmetry in both materials. However, in non-symmetric QWs as described in section 3.1, a spin orientation-induced CPGE has been observed, caused by the Rashba spin-orbit coupling due to built-in potential gradient in the QWs [23, 107]. A spin orientation-induced CPGE is most clearly seen for hh1-lh1 inter-subband absorption in (001)-oriented p-type QWs. For illumination with MIR radiation of the $\mathrm{CO}_{2}$ laser a current signal proportional to the helicity $P_{\text {circ }}$ is observed for oblique incidence (figure 13). For irradiation along $\langle 110\rangle$ as well as along $\langle 100\rangle$ crystallographic directions, the photocurrent flows perpendicular to the propagation direction of the incident light. Therefore only a transverse CPGE was observed. This means that the effect of a Dresselhaus-like $\boldsymbol{k}$-linear term yielding a longitudinal effect for $\langle 100\rangle$ is negligible [107]. The wavelength dependence of the photocurrent obtained between 9.2 and $10.6 \mu \mathrm{m}$ corresponds to the spectral behaviour of direct inter-subband absorption between the lowest heavy-hole and light-hole subbands measured in transmission (see figure 14). 


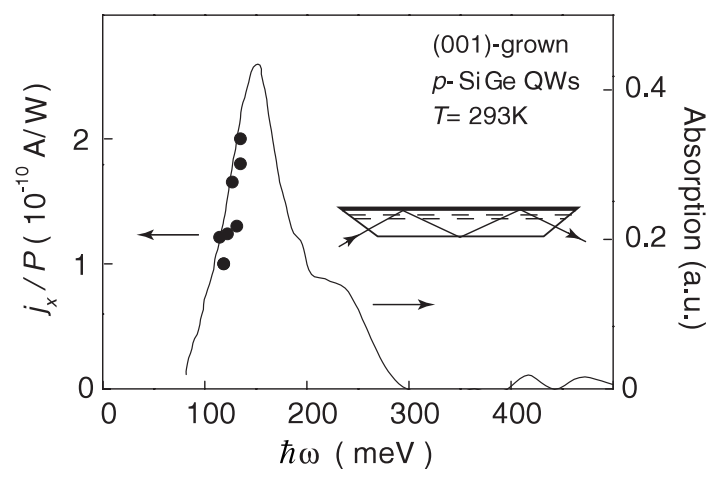

Figure 14. The spectral dependence of the spin orientation-induced CPGE (full dots) in (001)grown and asymmetrically doped $\mathrm{SiGe}$ QWs due to direct transitions between hh1 and lh1 valence subbands at room temperature. The full curve shows the absorption spectrum obtained at $10 \mathrm{~K}$. The absorption has been determined by transmission measurements making use of a multiple-reflection waveguide geometry shown in the inset.

In the FIR range a more complicated dependence of the current as a function of helicity has been observed. In (001)-grown asymmetric QWs as well as in (113)-grown samples, the dependence of the current on the phase angle $\varphi$ may be described by the sum of two terms; one of them is $\propto \sin 2 \varphi$ and the other $\propto \sin 2 \varphi \cos 2 \varphi$. In figure 15 experimental data and fits to these functions are shown for a step-bunched (001)-grown SiGe sample. The first term is due to the spin orientation-induced CPGE and the second term is caused by a LPGE [23] which will be discussed later (see section 5.1, equations (42)). For circularly polarized radiation the linear photogalvanic term $\sin 2 \varphi \cos 2 \varphi$ is equal to zero and the observed current is due to the spin orientation-induced CPGE only. We would like to point out that, in agreement with the symmetry, the same term may also be present in zinc-blende structure-based QWs but has not yet been detected. The CPGE and LPGE have different microscopic physical mechanisms. Variation of material parameters, excitation wavelengths, and temperature may change the relative strengths of these effects. For both spectral ranges, MIR and FIR, the angle of incidence dependence of the CPGE in SiGe structures is the same as shown above for zinc-blende structure-based materials.

The experimental results described so far are due to an imbalance of photoexcited spin-polarized electrons in $\boldsymbol{k}$-space yielding a CPGE. After momentum relaxation of the photoexcited carriers, the spin orientation-induced CPGE vanishes; however, a spin orientation may still be present if the spin relaxation time is longer than the momentum relaxation time. In such a case the spin-galvanic effect may contribute to the total current. In the next section we present experimental results demonstrating a pure spin-galvanic effect without admixture of a spin orientation-induced CPGE.

\subsection{The spin-galvanic effect}

4.2.1. The spin-galvanic effect in the presence of an external magnetic field. The spin photocurrent due to the spin-galvanic effect has been experimentally investigated by the method described in section 2.3.5 and is depicted in figure 7 [29, 30, 40, 41]. A homogeneous nonequilibrium spin polarization perpendicular to the plane of the (001)-grown QWs has been prepared by absorption of circularly polarized radiation normally incident. The measurements were carried out on n-type GaAs and InAs samples. In this experimental configuration the spin 


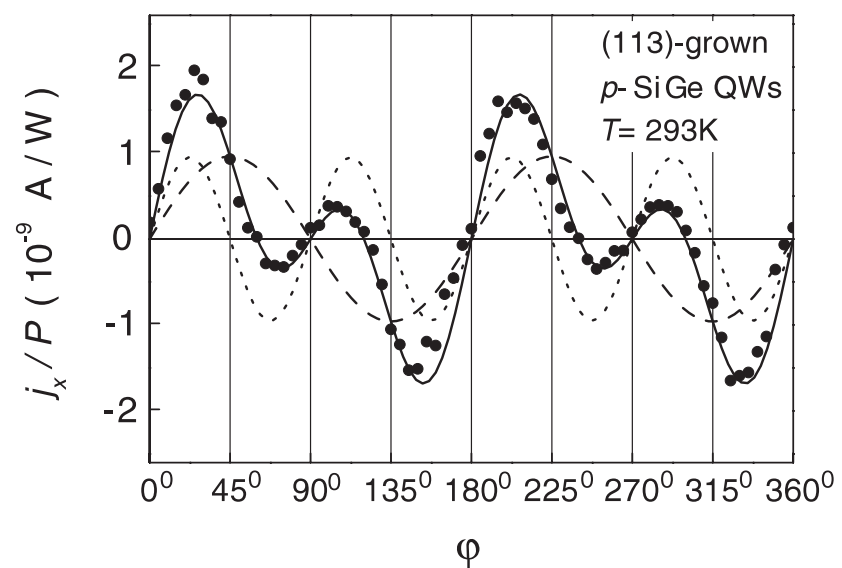

Figure 15. The photogalvanic current in (113)-grown SiGe QWs normalized by the light power $P$ as a function of the phase angle $\varphi$. The results were obtained at $\lambda=280 \mu \mathrm{m}$ for normal incidence of irradiation at room temperature. The full curve was fitted using the left-hand equations of equations (15) and (42) corresponding to the CPGE and the LPGE, respectively. Broken and dotted curves show $j_{x} \propto \sin 2 \varphi$ and $j_{x} \propto \sin 2 \varphi \cos 2 \varphi$, respectively.

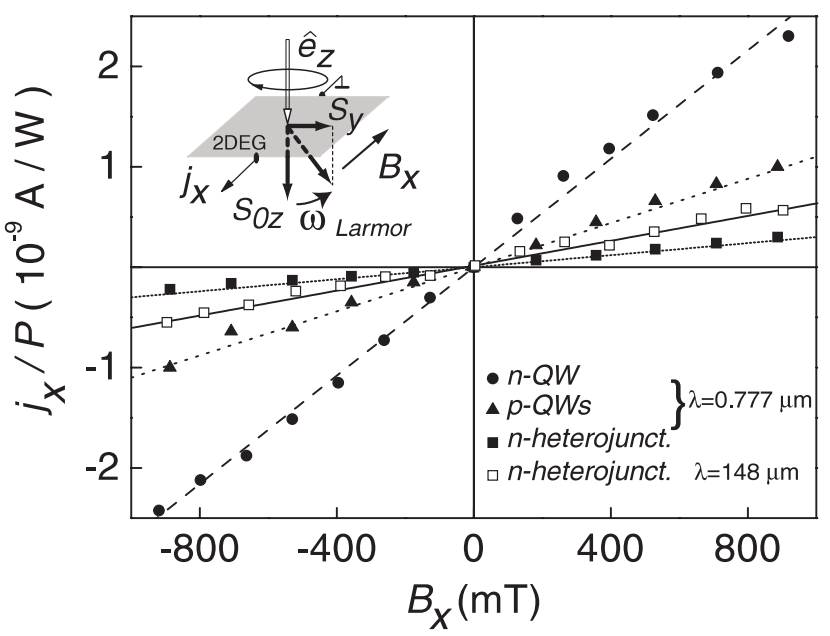

Figure 16. The spin-galvanic current $j_{x}$ normalized by $P$ as a function of the magnetic field $B$ for normally incident circularly polarized radiation at room temperature for various samples and wavelengths. Full symbols: $\lambda=0.777 \mu \mathrm{m}, P=100 \mathrm{~mW}$. Triangles, squares, and circles correspond to n-type and p-type multiple QWs, and an n-type GaAs/AlGaAs heterojunction, respectively. Open squares: an n-type GaAs/AlGaAs heterojunction, $\lambda=148 \mu \mathrm{m}, P=20 \mathrm{~kW}$. The inset shows the geometry of the experiment where $\hat{e}_{z}$ indicates the direction of the incoming light.

polarization does not yield an electric current. However, on applying an in-plane magnetic field, a spin-galvanic current has been observed in n-type materials for both visible and infrared radiation (figures 16-20).

For low magnetic fields $B$ where $\omega_{L} \tau_{s}<1$ holds, the photocurrent increases linearly as expected from equations (25) and (30). This is seen in the room temperature data of figures 16 and 17 as well as in the $4.2 \mathrm{~K}$ data in figure 18 for $B \leqslant 1 \mathrm{~T}$. The polarity of the current depends on the direction of the excited spins (see figures 17 and 18; $\pm z$-directions for right or 


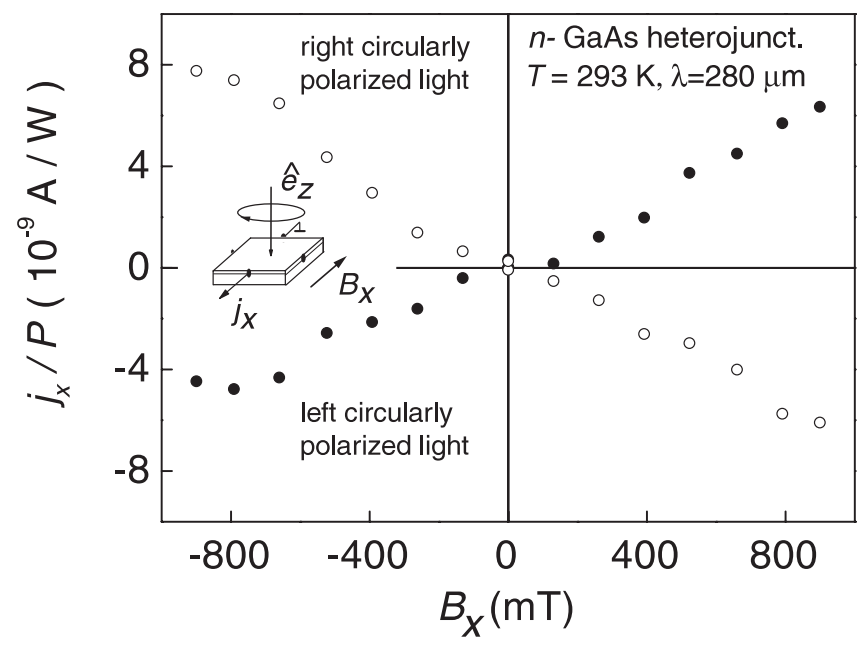

Figure 17. The magnetic field dependence of the spin-galvanic current, normalized by $P$, achieved by intra-subband transitions within the e 1 conduction subband caused by excitation with radiation of $\lambda=280 \mu \mathrm{m}$ wavelength. Results are plotted for an (001)-grown GaAs single heterojunction at room temperature.

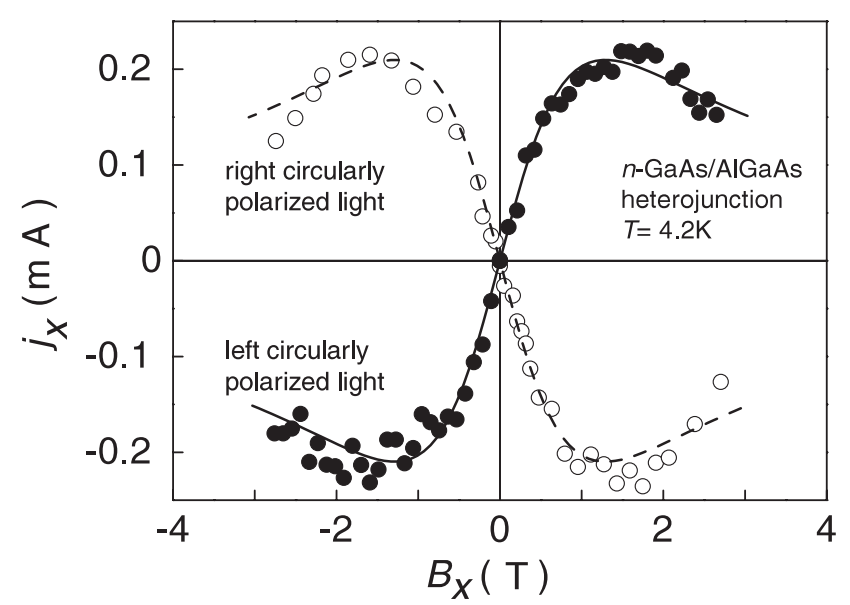

Figure 18. The spin-galvanic current $j_{x}$ as a function of the magnetic field $B$ for normally incident right-handed (open circles) and left-handed (solid circles) circularly polarized radiation at $\lambda=148 \mu \mathrm{m}$ and radiation power $20 \mathrm{~kW}$. Measurements are presented for an n-type GaAs/AlGaAs single heterojunction at $T=4.2 \mathrm{~K}$. Solid and dashed curves were fitted using equations (25) and (30) using the same value of the spin relaxation time $\tau_{s}$ and scaling of the ordinate.

left circularly polarized light, respectively) and on the direction of the applied magnetic field (see figures 16-19; $\pm B_{x}$-directions). For a magnetic field applied along $\langle 110\rangle$ the current is parallel (anti-parallel) to the magnetic field vector. For $B \|\langle 100\rangle$, both the transverse and the longitudinal effects are observed [30]. This observation as well as the helicity dependence of the photocurrent current shown in figure 19 are in good agreement with the phenomenological relation (see equations (32)).

Comparing the power sensitivity for visible and infrared excitation we find them to be of the same order of magnitude as seen in figure 16. However, we note that the current contribution 


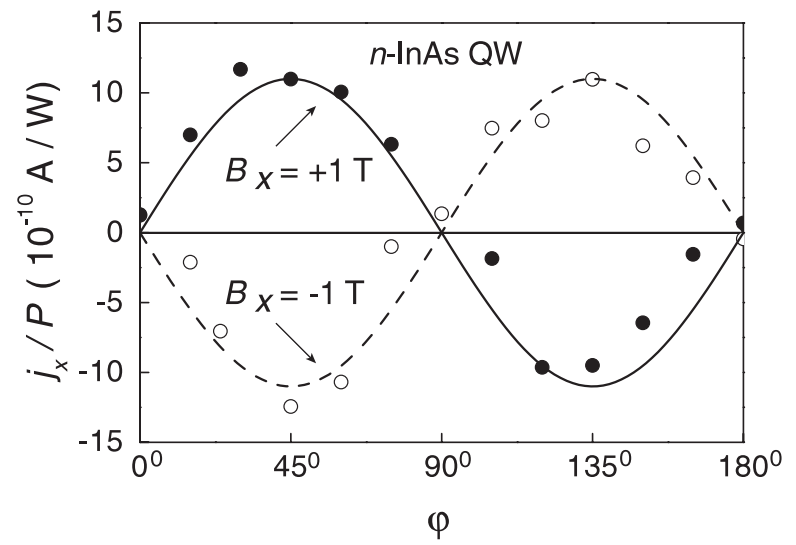

Figure 19. The spin-galvanic current normalized by $P$ as a function of the phase angle $\varphi$ in an (001)-grown n-type InAs QW of $15 \mathrm{~nm}$ width at $T=4.2 \mathrm{~K}$. The photocurrent excited by normally incident radiation of $\lambda=148 \mu \mathrm{m}$ is measured in the $x$-direction parallel (full circles) and antiparallel (open circles) to the in-plane magnetic field $B_{x}$. Solid and dashed curves were fitted using equations (32) using the same scaling of the ordinate.

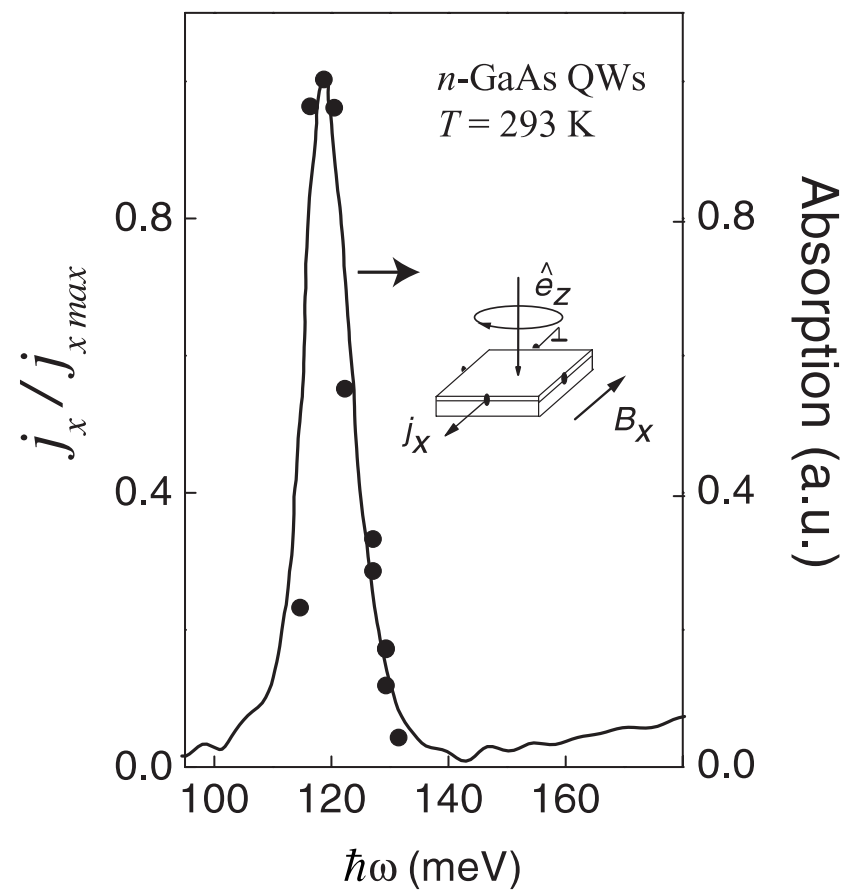

Figure 20. The spectral dependence of the spin-galvanic effect for (001)-grown n-type GaAs QWs of $8.2 \mathrm{~nm}$ width at room temperature. Data (dots) are presented for optical excitation by normally incident right-handed circularly polarized radiation. A magnetic field of $B_{x}=1 \mathrm{~T}$ was used. For comparison the absorption spectrum is shown, by the full curve.

per photon is larger by two orders of magnitude for inter-band excitation than for intra-subband absorption. This is due to the higher rate of spin generation by inter-band transitions. It may be even larger, since the current is somewhat reduced by photogenerated carriers in the semiinsulating substrate. For higher magnetic fields the current assumes a maximum and decreases 
upon further increase of $B$, as shown in figure 18. This drop of the current is ascribed to the Hanle effect [3]. The experimental data are well described by equations (25) and (30). The observation of the Hanle effect demonstrates that free carrier intra-subband transitions can polarize the spins of electron systems. The measurements allow us to obtain the spin relaxation time $\tau_{s}$ from the peak position of the photocurrent, where $\omega_{L} \tau_{s}=1$ holds [29].

In p-type GaAs QWs under infrared excitation causing spin polarization of holes only, no spin-galvanic effect could be detected $[29,30]$. In contrast to the case for infrared experiments, a current signal has been detected in p-type samples for visible excitation which polarizes both electrons and holes (see figure 16). This current is due to the spin polarization of electrons only, which are in this case the minority carriers generated by inter-band excitation. The spin-galvanic effect in p-type material under inter- or intra-subband excitation could not be observed because of the experimental procedure, which makes use of the Larmor precession to obtain an in-plane spin polarization. This is because the in-plane $g$-factor for heavy holes is very small [108], which makes the effect of the magnetic field negligible [29]. This result does not exclude the possibility of a spin-galvanic effect in p-type materials, which might be observable by means of hole injection with spins in the plane of the QW.

Spin photocurrents due to the spin-galvanic effect have been recorded for inter-band, inter-subband, as well as for intra-subband transitions [29-31].

Inter-band transitions in $\mathrm{n}$ - and p-type GaAs have been investigated using circularly polarized light from a Ti:sapphire laser at $\lambda=0.777 \mu \mathrm{m}$. In this experiment, electrons are excited from the valence band to the conduction band yielding a spin polarization in the conduction band by the selection rules.

Direct inter-subband transitions have been achieved in GaAs QWs of widths 8.2 and $8.6 \mathrm{~nm}$ for absorption of radiation in the range of 9-11 $\mu \mathrm{m}$ wavelength [40,41]. Applying MIR radiation from a $\mathrm{CO}_{2}$ laser, a spin-galvanic current has been observed for normal incidence of radiation. In contrast to the case for the spin orientation-induced CPGE, the wavelength dependence of the spin-galvanic effect obtained between 9.2 and $10.6 \mu \mathrm{m}$ repeats the spectral behaviour of direct inter-subband absorption (see figure 20). This observation is in agreement with the mechanism of the spin-galvanic effect and the microscopic theory presented in section 2.3.3. The occurrence of a spin-galvanic current requires only a spin polarization in the lower subband and asymmetric spin relaxation. In the present case the spin orientation is generated by resonant spin-selective optical excitation followed by spinnon-specific thermalization. Therefore the magnitude of the spin polarization and hence the current depends on the absorption strength but not on the momentum $k$ of optical transition as in the case of the CPGE described in section 2.2.1.

We would like to emphasize that spin-sensitive e1-e2 inter-subband transitions in n-type QWs have been observed for normal incidence when there is no component of the electric field of the radiation normal to the plane of the QWs. Generally it is believed that inter-subband transitions in n-type QWs can only be excited by infrared light polarized in the growth direction $z$ of the QWs [14]. Furthermore, such transitions are spin insensitive and, hence, do not lead to optical orientation. Since the argument leading to these selection rules is based on the effective mass approximation in a single-band model, the selection rules are not rigorous. The mechanism which leads to spin orientation in this geometry will be discussed in section 4.3.1.

For indirect transitions the spin-galvanic effect, as in the case of the spin orientationinduced CPGE, has been obtained in n-type GaAs and InAs QWs using FIR radiation (see figures 16-19). The presence of the spin-galvanic effect, which is due to spin orientation excitable at MIR and FIR wavelengths, gives clear evidence that direct inter-subband and Drude absorption of circularly polarized radiations results in spin orientation. The mechanism of this spin orientation is not obvious and will be introduced in section 4.3.2. 
4.2.2. The spin-galvanic effect for optical excitation without external fields. In the experiments described above an external magnetic field was used for reorientation of an optically generated spin polarization. The spin-galvanic effect can also be observed for optical excitation alone, without application of an external magnetic field. The necessary in-plane component of the spin polarization is obtained for oblique incidence of the exciting circularly polarized radiation. In this case, however, a spin orientation-induced CPGE may also occur, interfering with the spin-galvanic effect. Nevertheless, a pure spin-galvanic current may be obtained for inter-subband transitions in n-type GaAs QWs [31]. As shown above, the spectrum of the CPGE changes sign and vanishes at the centre of resonance [24] (see section 4.1.2 and equations (22) and (23)). In contrast, the optically induced spin-galvanic current is proportional to the absorbance (equations (29)) and, hence, assumes a maximum at the centre of the resonance $[40,41]$ (see section 4.2.1). Thus, if a measurable helicity-dependent current is present at the centre of the resonance, it must be attributed to the spin-galvanic effect.

These experiments have been carried out making use of the spectral tunability of the free electron laser 'FELIX' [103]. The photon energy dependence of the current was measured for incidence in two different planes with components of propagation along the $x$-and $y$-directions. In figure 21 the observed current, for both directions, is plotted as a function of photon energy $\hbar \omega$ for $\sigma_{+}$-radiation together with the absorption spectrum. It can be seen that for a current along $x \|[1 \overline{1} 0]$, the spectral shape is similar to the derivative of the absorption spectrum, and in particular there is a change of sign which occurs at the line centre of the absorption. When the sample was rotated by $90^{\circ}$ about $z$, the sign change of the current, now along $y \|[110]$, disappears and its spectral shape follows more closely the absorption spectrum.

The spectral inversion of sign of the photocurrent in the $x$-direction indicates the CPGE, which is proportional to the derivative of the absorption spectrum (see equations (22) and (23)). In contrast with the case for the CPGE, the sign of the spin-galvanic current does not depend on the wavelength (see section 4.2.1 and [40,41]). This can be seen from figure 5(b) which illustrates the origin of the spin-galvanic effect. All that is required is a spin orientation of the lower subband, and asymmetrical spin relaxation then drives a current [29]. In our case the spin orientation is generated by resonant spin-selective optical excitation followed by spin-nonspecific thermalization. The magnitude of the spin polarization, and hence the current, depends on the initial absorption strength but not on the momentum $k$ of the transition. Therefore there is no sign change and the shape of the spectrum follows the absorption (see equations (29) and $[31,40,41])$. The lack of a sign change for current along $y \|[110]$ in the experiment shows that the spin-galvanic current dominates for this orientation.

The non-equivalence of the two orientations [110] and [1 $\overline{10}]$ is caused by the interplay of the BIA and SIA terms in the Hamiltonian when rotating the wavevector in the QW plane. Both currents, the CPGE and the spin-galvanic current, are due to the spin splitting of subbands in $\boldsymbol{k}$-space described by equation (1). The pseudo-tensors $\gamma$ and $Q$ determining the current are related to the transposed pseudo-tensor $\boldsymbol{\beta}$. They are subjected to the same symmetry restrictions, so their irreducible components differ only by scalar factors. In $\mathrm{C}_{2 \mathrm{v}}$ symmetry, usually $\beta_{y x} \neq \beta_{x y}$, and it is reasonable to introduce symmetric and anti-symmetric tensor components $\beta_{B I A}^{(\nu)}=(1 / 2)\left(\beta_{x y}^{(v)}+\beta_{y x}^{(v)}\right)$ and $\beta_{S I A}^{(v)}=(1 / 2)\left(\beta_{x y}^{(v)}-\beta_{y x}^{(v)}\right)$, where $v=1,2$ indicates the 1 and 2 subbands respectively. $\beta_{B I A}^{(\nu)}$ and $\beta_{S I A}^{(v)}$ result from BIA and from SIA, respectively.

As discussed above and sketched in figure 5, both the CPGE and the spin-galvanic currents, say in the $x$-direction, are caused by the band splitting in the $k_{x}$-direction and therefore are proportional to $\beta_{y x}$ (for current in the $y$-direction one should interchange the indices $x$ and $y$ ). 


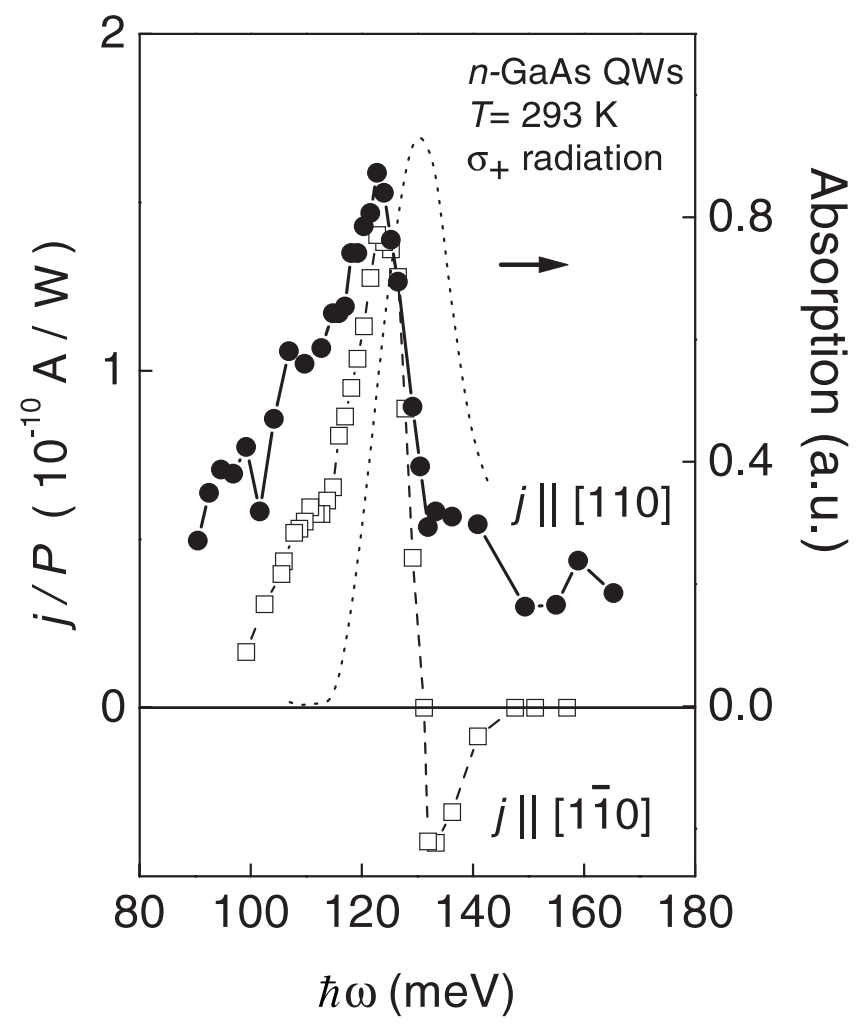

Figure 21. The photocurrent in QWs normalized by the light power $P$ for right-handed circularly polarized radiation obliquely incident on n-type (001)-grown GaAs/AlGaAs QWs of $8.2 \mathrm{~nm}$ width at $T=293 \mathrm{~K}$ as a function of the photon energy $\hbar \omega$. Circles: current in the [110] direction in response to irradiation parallel to [1ㅣㅣ]. Rectangles: current in the [1 10$]$ direction in response to irradiation parallel to [110]. The dotted curve shows the absorption measured using a Fourier transform spectrometer.

Then the currents in the $x$ - and $y$-directions read

$j_{x}=A_{C P G E}\left[\left(\beta_{B I A}^{(1)}-\beta_{S I A}^{(1)}\right)-\left(\beta_{B I A}^{(2)}-\beta_{S I A}^{(2)}\right)\right] P_{c i r c} \hat{e}_{y}+A_{S G E}\left(\beta_{B I A}^{(1)}-\beta_{S I A}^{(1)}\right) S_{y}$

and

$j_{y}=A_{C P G E}\left[\left(\beta_{B I A}^{(1)}+\beta_{S I A}^{(1)}\right)-\left(\beta_{B I A}^{(2)}+\beta_{S I A}^{(2)}\right)\right] P_{c i r c} \hat{e}_{x}+A_{S G E}\left(\beta_{B I A}^{(1)}+\beta_{S I A}^{(1)}\right) S_{x}$,

where $A_{C P G E}$ and $A_{S G E}$ are factors related to $\gamma$ and $Q$, respectively, and the subscript $S G E$ indicates the spin-galvanic effect.

In the present case, $S$ is obtained by optical orientation; its sign and magnitude are proportional to $P_{c i r c}$ and it is oriented along the in-plane component of $\hat{e}$. The magnitude of the CPGE is determined by the values of $\boldsymbol{k}$ in the initial and final states, and hence depends on the spin splittings $\beta_{B I A}$ and $\beta_{S I A}$ of the e1 and e2 subbands. In contrast, the spin-galvanic effect is due to relaxation between the spin states of the lowest subband and hence depends only on $\beta_{B I A}$ and $\beta_{S I A}$ for e1.

The equations above show that in the directions $y$ and $x$ the spin-galvanic effect and the CPGE are proportional to terms involving the sum and the difference respectively of BIA and SIA terms. For our sample it appears that in the case where they add, the spin-galvanic effect dominates over the CPGE, consistently with the lack of sign change for the current 
along the $y$-direction in figure 21. Conversely, when the difference of BIA and SIA terms is concerned, the spin-galvanic effect is suppressed and the CPGE dominates. We emphasize that at the maximum of absorption, where the spin orientation-induced CPGE is equal to zero for both directions, the current obtained is caused solely by the spin-galvanic effect.

\subsection{Monopolar spin orientation}

Absorption of circularly polarized light in semiconductors may result in spin polarization of photoexcited carriers. While this phenomenon of optical orientation caused by inter-band transitions in semiconductors has been known of for a long time [8, 109-111] and has been widely studied [3], it is not obvious that free carrier absorption due to inter-subband and intrasubband transitions can also result in a spin polarization. Observation of a spin polarizationinduced CPGE and the spin-galvanic effect in the MIR and FIR spectral ranges unambiguously demonstrates that spin orientation may be achieved due to free carrier absorption. This optical orientation may be referred to as 'monopolar' [36] because photon energies are much less than the fundamental energy gap and only carriers of one type, electrons or holes, are excited. Here we consider mechanisms of monopolar optical orientation due to direct inter-subband transitions as well as by Drude-like intra-subband absorption for n- and p-type QWs based on zinc-blende structure semiconductors.

Monopolar spin orientation in n-type QWs becomes possible if an admixture of valence band states to the conduction band wavefunction and the spin-orbit splitting of the valence band are taken into account $[40,41]$. We emphasize that the rate of spin generation under monopolar optical orientation depends strongly on the energy of the spin-orbit splitting of the valence band, $\Delta_{s o}$. This is due to the fact that the $\Gamma_{8}$ valence band and the $\Gamma_{7}$ spin-orbit split-off band contribute to the matrix element of spin-flip transitions with opposite signs. For p-type QWs, analogous mechanisms are responsible for the spin orientation.

The rate of generation of the electron spin polarization $S$ due to optical excitation can be written as

$$
\dot{S}=s(\eta I / \hbar \omega) P_{c i r c},
$$

where $s$ is the average electron spin generated per absorbed photon of circularly polarized radiation, and $\eta$ is the fraction of the energy flux absorbed in the QW.

4.3.1. Direct transitions between size-quantized subbands. As equation (35) shows, the spin generation rate $\dot{S}$ is proportional to the absorbance $\eta_{12}$. In order to explain the observed spin orientation for inter-subband transitions between e 1 and e 2 subbands in n-type QWs and, in particular, the absorption of light polarized in the plane of a $\mathrm{QW}$, the $\boldsymbol{k} \cdot \boldsymbol{p}$ admixture of valence band states to the conduction band wavefunctions has to be taken into account [40, 41]. Calculations yield that inter-subband absorption of circularly polarized light propagating along $z$ induces only spin-flip transitions resulting in $100 \%$ optical orientation of photoexcited carriers, i.e. $s=1$. In this geometry the fraction of the energy flux absorbed in the QW by transitions from the first subband $\mathrm{e} 1$ to the second subband $\mathrm{e} 2$ has the form

$$
\eta_{12}=\frac{128 \alpha^{*}}{9 n} \frac{\Delta_{s o}^{2}\left(2 \varepsilon_{g}+\Delta_{s o}\right)^{2}\left(\varepsilon_{e 2}-\varepsilon_{e 1}\right) \varepsilon_{e 1}}{\varepsilon_{g}^{2}\left(\varepsilon_{g}+\Delta_{s o}\right)^{2}\left(3 \varepsilon_{g}+2 \Delta_{s o}\right)^{2}} \frac{\hbar^{2} n_{s}}{m_{e 1}^{*}} \delta\left(\hbar \omega-\varepsilon_{e 1}+\varepsilon_{e 2}\right),
$$

where $\alpha^{*}$ is the fine-structure constant, $n$ is a refraction index, $n_{s}$ is a free carrier density, and $\varepsilon_{e 1}$ and $\varepsilon_{e 2}$ are the energies of the size-quantized subbands e1 and e2, respectively. The $\delta$-function describes the resonant behaviour of the inter-subband transitions. 
In p-type QWs, optical orientation is caused by heavy-hole-to-light-hole absorption of circularly polarized radiation and occurs for transitions with in-plane wavevector $k \neq 0$ due to the mixing of heavy-hole and light-hole subbands [112].

4.3.2. Drude absorption due to indirect intra-subband transitions. In the FIR range where the photon energy is not enough for a direct inter-subband transition in n- or in p-type samples, the absorption of light by free carriers is caused by indirect intra-subband transitions where the momentum conservation law is satisfied due to emission or absorption of acoustic or optical phonons, static defects, etc (Drude-like absorption). We assume that the carriers occupy the e1 subband. The intra-subband optical transitions in QWs involving both the electron-photon interaction and momentum scattering are described as second-order processes with virtual transitions via intermediate states. The compound matrix elements for such transitions with the initial and final states in the same band have the standard form [40]

$$
M_{c m_{s}^{\prime} k^{\prime} \leftarrow c m_{s} k}=\sum_{\nu}\left(\frac{V_{c m_{s}^{\prime} k^{\prime}, \nu k} R_{\nu k, c m_{s} k}}{\varepsilon_{\nu k}-\varepsilon_{c k}-\hbar \omega}+\frac{R_{c m_{s}^{\prime} k^{\prime}, \nu k^{\prime}} V_{\nu k^{\prime}, c m_{s} k}}{\varepsilon_{\nu k^{\prime}}-\varepsilon_{c k} \pm \hbar \Omega_{k-k^{\prime}}}\right) .
$$

Here $\varepsilon_{c k}, \varepsilon_{c \boldsymbol{k}^{\prime}}$, and $\varepsilon_{v}$ are the electron energies in the initial $\left|c, m_{s}, \boldsymbol{k}\right\rangle$, final $\left|c, m_{s}^{\prime}, \boldsymbol{k}^{\prime}\right\rangle$, and intermediate $|v\rangle$ states, respectively, $m_{s}$ is the spin index, $R$ is the matrix element for electron interaction with the electromagnetic wave, $V$ is the matrix element for electron-phonon or electron-defect interaction, and $\hbar \Omega_{k-k^{\prime}}$ is the energy of the phonon involved. The signs \pm in equation (37) correspond to emission and absorption of phonons. A dominant contribution to the optical absorption is caused by processes with intermediate states in the same subband. This is the channel that determines the coefficient of intra-subband absorbance, $\eta$. However, such transitions conserve the electronic spin and, hence, do not lead to an optical orientation.

In order to obtain optical orientation due to intra-subband transitions for normal incidence, we consider virtual inter-band transitions with intermediate states in the valence band [40,41]. Figure 22 shows schematically the spin orientation in intra-band absorption of right-handed circularly polarized light $\left(\sigma_{+}\right)$for normal incidence. Because of the dipole selection rules for inter-band optical transitions, the electron transitions with spin reversal from $m_{s}=-1 / 2$ to $+1 / 2$ are possible via intermediate states in the light-hole and spin-orbit-split subbands, while the opposite processes, $+1 / 2 \rightarrow-1 / 2$, are forbidden. As a result, spin orientation of electrons occurs. At oblique incidence, the transitions via heavy-hole subbands also contribute to the optical orientation.

For this particular mechanism of monopolar optical orientation, one can derive the following expression for the spin generated per absorbed photon of e.g. right-handed circularly polarized radiation:

$$
s \propto \frac{V_{c v}^{2}}{V_{c}^{2}} \frac{\hbar \omega \Delta_{s o}^{2}}{\varepsilon_{g}\left(\varepsilon_{g}+\Delta_{s o}\right)\left(3 \varepsilon_{g}+2 \Delta_{s o}\right)} .
$$

Here $V_{c}$ and $V_{c v}$ are the intra-subband and inter-band matrix elements, respectively, and they depend on the mechanism of momentum scattering. Acoustic phonon-assisted and static impurity processes are considered in [40, 41].

\subsection{The spin-controlled non-linearity of the spin orientation-induced CPGE}

Here we discuss the non-linear behaviour of the spin polarization-induced CPGE. It was observed in $[37,38]$ that the photocurrent saturates with increasing light intensity. In fact, in this case, the photogalvanic current normalized by the radiation intensity $I$ is proportional to the absorbance [21] and reflects the power dependence of the absorption coefficient. 
(a)

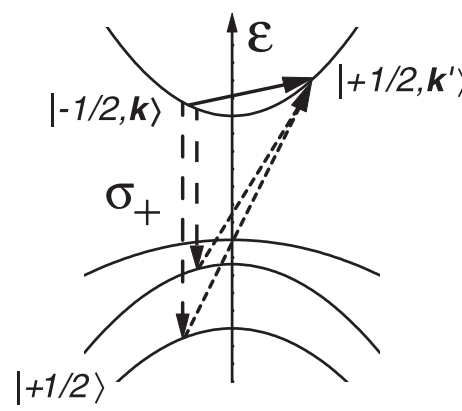

(b)

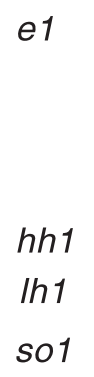

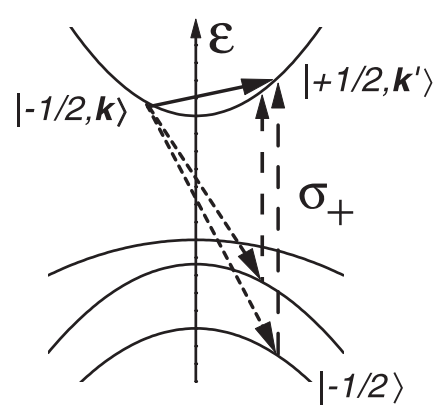

Figure 22. A sketch of the indirect intra-subband optical transitions (solid arrows) with intermediate states in the valence band. Dashed and dotted arrows indicate the electron-photon interaction and the electron momentum scattering.

The saturation effect on p-doped QW structures was investigated for direct inter-subband optical transitions. The basic physics of spin-sensitive bleaching of absorption is sketched in figure 23. Excitation with FIR radiation results in direct transitions between heavy-hole hh1 and light-hole $\mathrm{lh} 1$ subbands. This process depopulates and populates selectively spin states in hh 1 and lhl subbands. The absorption is proportional to the difference in population of the initial and final states. At high intensities the absorption decreases, since the photoexcitation rate becomes comparable to the rate of non-radiative relaxation to the initial state. By the selection rules, spins of only one type are involved in the absorption of circularly polarized light. Thus the absorption bleaching of circularly polarized radiation is governed by the energy relaxation of photoexcited carriers and spin relaxation in the initial state (see figure 23(b)). These processes are characterized by energy and spin relaxation times $\tau_{e}$ and $\tau_{s}$, respectively. We note that during energy relaxation to the initial state in hh1, the holes lose their photoinduced orientation due to rapid relaxation [113]. Thus, spin orientation occurs in the initial subband hh1, only. In contrast to the case for circularly polarized light, absorption of linearly polarized light is not spin selective and the saturation is controlled by the energy relaxation only (see figure 23(c)). If $\tau_{s}$ is larger than $\tau_{e}$, the bleaching of the absorption becomes spin sensitive and the saturation intensity of the circularly polarized radiation drops below the value for linear polarization.

The difference between the absorption bleaching for circularly polarized radiation and that for linearly polarized radiation has been observed experimentally employing the spin orientation-induced CPGE (see section 2.2) and the LPGE (see section 5.1) [38]. Figure 24 shows that the photocurrent $j_{x}$ measured for p-type GaAs QWs depends on the intensity $I$ as $j_{x} \propto I /\left(1+I / I_{s}\right)$, where $I_{s}$ is the saturation intensity. It has been shown that the saturation intensities $I_{s}$ for circularly polarized radiation are generally smaller than that for linearly polarized radiation (figure 25). The non-linear behaviour of the photogalvanic current has been analysed in terms of excitation-relaxation kinetics taking into account both optical excitation and non-radiative relaxation processes. It has been shown that the photocurrent $j_{L P G E}$ induced by linearly polarized radiation is described by $j_{L P G E} / I \propto\left(1+I / I_{s e}\right)^{-1}$, where $I_{s e}$ is the saturation intensity controlled by the energy relaxation of the hole gas. The photocurrent $j_{C P G E}$ induced by circularly polarized radiation is proportional to $\left(1+I\left(1 / I_{s e}+1 / I_{S S}\right)\right)^{-1}$ where $I_{s s}=\hbar \omega p_{s} /\left(\alpha_{0} \mathrm{~d} \tau_{s}\right)$ is the saturation intensity controlled by hole spin relaxation, $\alpha_{0}$ is the absorption coefficient at low intensities and $d$ is the period of multiple QW structure. 
(a)

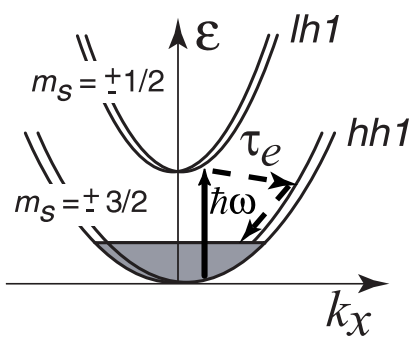

(b) circularly polarized

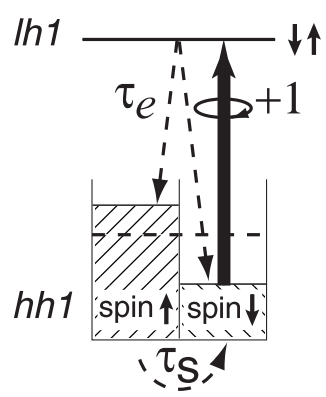

(c) linearly polarized radiation

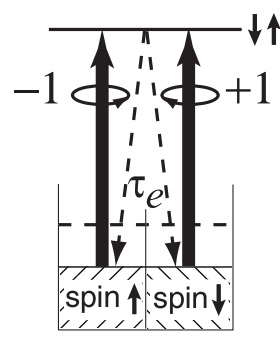

Figure 23. The microscopic picture of spin-sensitive bleaching: (a) a sketch of direct optical transitions (full line) between hh1 and lh1 in p-type GaAs/AlGaAs QWs; ((b), (c)) sketches of the process of bleaching for circularly and linearly polarized radiation, respectively. Dashed arrows indicate energy $\left(\tau_{e}\right)$ and spin $\left(\tau_{s}\right)$ relaxation.

Using the experimentally obtained $I_{S S}$ together with the absorption coefficient $\alpha_{0}$, calculated following [114], spin relaxation times $\tau_{s}$ have been derived (see figure 26) [38]. We note that in the definition of $I_{s s}$ it was assumed that the spin selection rules are fully satisfied at the transition energy. This is the case for optical transitions occurring close to $k=0$ [113] realized in the above experiment. If this is not the case, the mixture of heavy- and light-hole subbands reduces the strength of the selection rules [73] and therefore reduces the efficiency of the spin orientation. The mixing yields a multiplicative factor in $I_{s s}$, increasing the saturation intensity for constant spin relaxation time [37]. In [115] a substantial lowering of $I_{s s}$ with decreasing QW width was observed. This observation may indicate much slower spin relaxation times for holes in narrow QWs, as obtained theoretically in [113].

This result shows that spin relaxation of holes may be obtained by investigation of the CPGE as a function of the radiation intensity. A substantial proportion of investigations of the spin lifetime in semiconductor devices are based on optical spin orientation by interband excitation and further tracing of the kinetics of polarized photoluminescence (for a review see $[1,3,116,117])$. These studies give important insight into the mechanisms of spin relaxation of photoexcited free carriers. In contrast to these methods of optical spin orientation, the monopolar spin orientation method allows one to study spin relaxation without electron-hole interaction and exciton formation in conditions close to those for electric spin injection [29, 38, 39]. Spin photocurrents provide experimental access to such investigations.

\section{Spin-independent photocurrents under homogeneous excitation}

When investigating spin photocurrents, one should take it into account that optical excitation may generate other currents which are not the result of spin orientation. Spin photocurrents can be recognized by their dependence on the helicity of the exciting radiation. Indeed, only spin photocurrents change their direction when the state of polarization of radiation is switched from right to left handed or vice versa. This allows one to extract the spin photocurrent contribution from total photocurrents under visible excitation when spin-independent photocurrents, such as in the case of the Dember effect or the photovoltaic effect at contacts, may occur; these are substantially larger than spin photocurrents. In this situation the periodic modulation of the state of polarization and synchronous detection of the current constitutes a convenient technique 


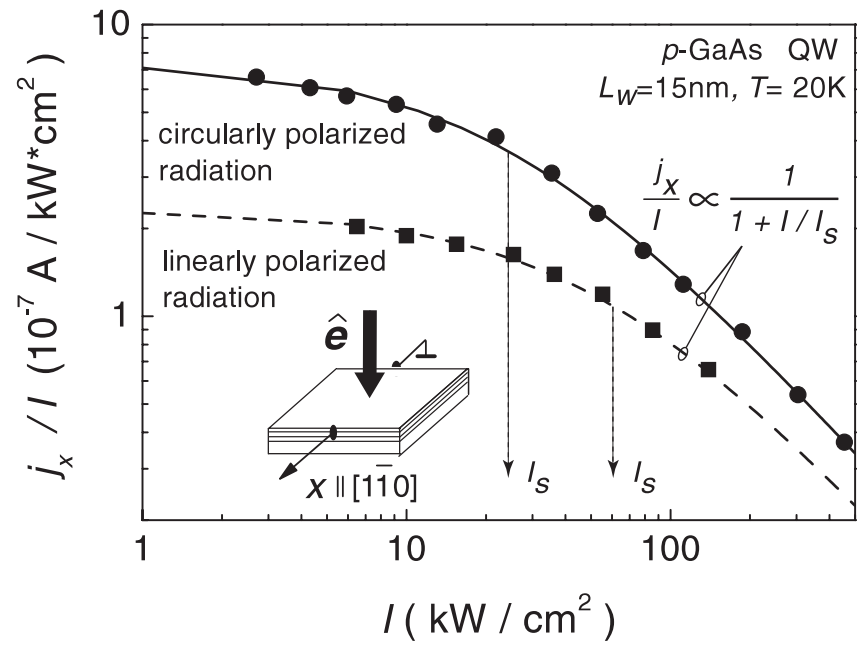

Figure 24. CPGE and LPGE currents $j_{x}$ normalized by the intensity $I$ as a function of $I$ for circularly and linearly polarized radiation of $\lambda=148 \mu \mathrm{m}$, respectively. The inset shows the geometry of the experiment. The current $j_{x}$ is measured along [110] for radiation normally incident on p-type (113)A-grown GaAs QW with $L_{W}=15 \mathrm{~nm}$ at $T=20 \mathrm{~K}$. The LPGE was obtained with the electric field vector $\boldsymbol{E}$ oriented at $45^{\circ}$ to the $x$-direction (see equations (44)). The measurements were fitted to $j_{x} / I \propto 1 /\left(I+I / I_{s}\right)$ with one parameter $I_{s}$ for each state of polarization (full curve: circular; broken curve: linear).

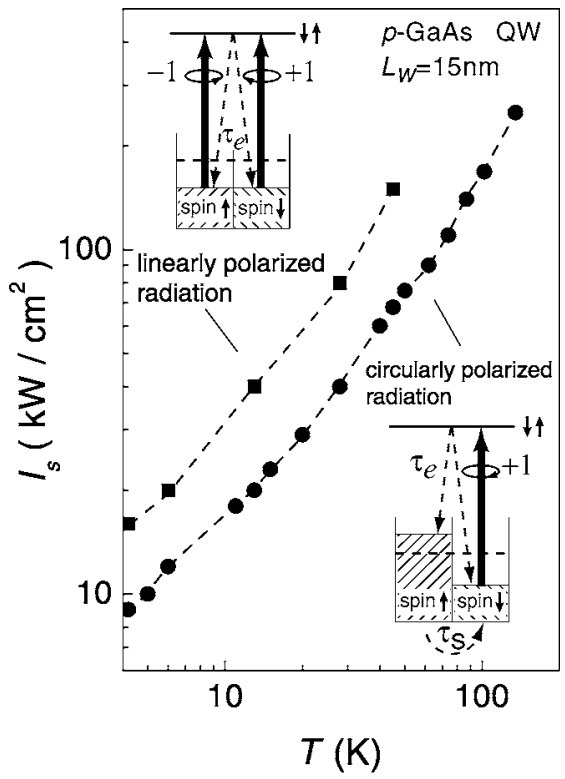

Figure 25. The temperature dependence of the saturation intensity $I_{s}$ for linearly and circularly polarized radiation of $\lambda=148 \mu \mathrm{m}$. The dependence is shown for a p-type GaAs/AlGaAs (113)Agrown sample with a single QW of $L_{W}=15 \mathrm{~nm}$ width. The free carrier density and the mobility were $1.66 \times 10^{11} \mathrm{~cm}^{-2}$ and $6.5 \times 10^{5} \mathrm{~cm}^{2} \mathrm{~V}^{-1} \mathrm{~s}^{-1}$, respectively.

for measuring spin photocurrents in itself. In the infrared spectral range these strong spinindependent photocurrents do not occur. However, there are two other sources of photocurrents 


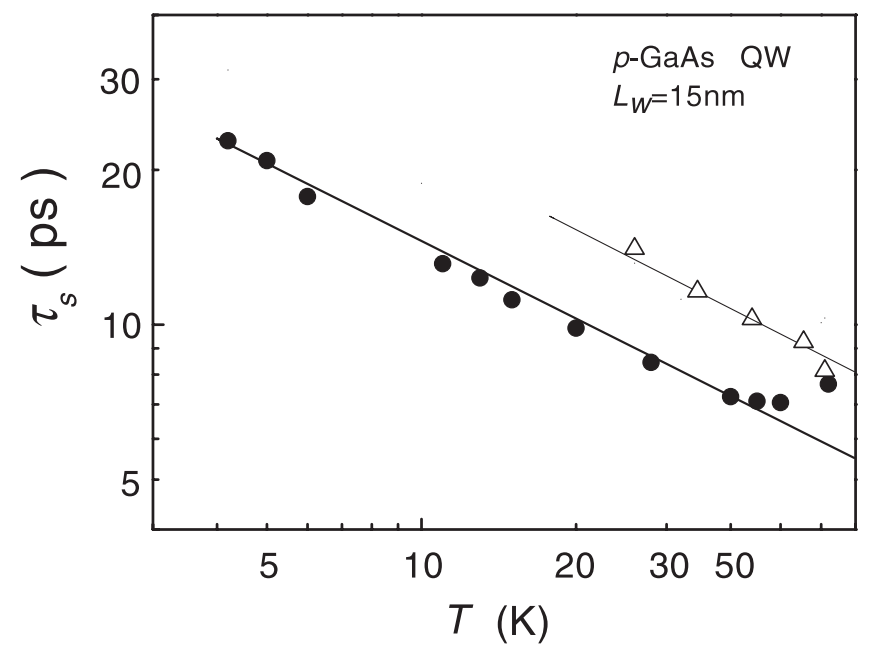

Figure 26. Experimentally determined spin relaxation times $\tau_{s}$ for holes in p-type GaAs/AlGaAs QWs as a function of temperature $T$. Open triangles and full dots correspond to (113)A-grown $15 \mathrm{~nm}$ single and multiple (twenty) QWs, respectively. The free carrier densities for all samples were about $2 \times 10^{11} \mathrm{~cm}^{-2}$ for each QW.

under homogeneous excitation which occur simultaneously and may be of the same order of magnitude as spin photocurrents, but again do not require spin orientation. These are the LPGE [13, 14] and the photon drag effect [118, 119]. These photocurrents are not changed in sign or amplitude if the polarization is switched from $\sigma_{+}$to $\sigma_{-}$. Furthermore, both effects, the LPGE and the photon drag effect, depend on the symmetry of the material in a different way to spin photocurrents. Therefore in most cases it is possible to choose a crystallographic orientation and an experimental geometry where only spin photocurrents occur. In other cases the helicity dependence of the photocurrents allows one to distinguish between spin photocurrents and spin-independent photocurrents. On the basis of the phenomenology, we discuss spin-independent photocurrents in the following and present some experimental examples.

By general symmetry arguments it can be shown that these spin-independent photocurrents are given by

$$
j_{\lambda}=\sum_{\mu \nu} \chi_{\lambda \mu \nu}\left(E_{\mu} E_{\nu}^{*}+E_{\nu} E_{\mu}^{*}\right) / 2+\sum_{\delta \mu \nu} T_{\lambda \delta \mu \nu} q_{\delta} E_{\mu} E_{\nu}^{*} .
$$

where $\chi_{\lambda \mu \nu}$ and $T_{\lambda \delta \mu \nu}$ are components of a third-rank and a fourth-rank tensor, respectively, and $q_{\delta}$ is the wavevector of the radiation in the sample.

The first term on the right-hand side of equation (39) is called the LPGE because it is independent of the sign of the circular polarization and is usually measured using linearly polarized radiation. The second term represents the photon drag effect, which yields a current due to momentum transfer from photons to electrons.

\subsection{The linear photogalvanic effect}

The LPGE arises in homogeneous samples under homogeneous excitation due to an asymmetry of the scattering of free carriers on phonons, static defects, or other carriers in noncentrosymmetric media (for a review see $[13,14]$ ). The LPGE was observed in some insulators as early as the 1950s, but was correctly identified as a new phenomenon only in [120] and 
theoretically treated in [121]. For bulk materials such as GaAs, the LPGE was studied in great detail (for a review see [11-14]). It has also been considered for and observed in lowdimensional structures such as MOSFET Si structures [122, 123], III-V QWs [10, 21, 35, 124], and asymmetric SiGe QWs [23]. Microscopically, the LPGE current consists of a so-called ballistic contribution and a shift contribution [125-127]. The LPGE was successfully applied as a fast detector of the degree of linear polarization [128, 129].

As shown by equation (39), the linear photogalvanic current is linked to the symmetrized product $E_{\mu} E_{v}^{*}$ by a third-rank tensor $\chi_{\lambda \mu \nu}$ which in turn is symmetric in the last two indices. Therefore $\chi_{\lambda \mu \nu}$ is isomorphic to the piezoelectric tensor and may have non-zero components in media lacking a centre of symmetry. Note that, in contrast to the case for spin photocurrents, gyrotropy is not necessary. In zinc-blende structure-based QWs of $\mathrm{C}_{2 \mathrm{v}}$ symmetry, taking into account only the LPGE term of equation (39), we get

$$
j_{L P G E, x}=\chi_{x x z}\left(E_{x} E_{z}^{*}+E_{z} E_{x}^{*}\right), \quad j_{L P G E, y}=\chi_{y y z}\left(E_{y} E_{z}^{*}+E_{z} E_{y}^{*}\right) .
$$

For higher-symmetry QWs of point group $\mathrm{D}_{2 \mathrm{~d}}$, the coefficients are linearly dependent and $\chi_{x x z}=-\chi_{y y z}$. Equations (40) show that the LPGE occurs only for oblique incidence of radiation, because a component of the electric field, $E_{z}$, along the $z$-axis is required.

We assume now irradiation of a $\mathrm{QW}$ of $\mathrm{C}_{2 \mathrm{v}}$ symmetry with the plane of incidence parallel to $(y z)$. For linearly polarized light with an angle $\alpha$ between the plane of polarization defined by the electric field vector and the $x$-coordinate parallel to [1 $\overline{10}$ ], the LPGE current is given by

$$
j_{L P G E, x}=\chi_{x x z} \hat{e}_{y} E_{0}^{2} \sin 2 \alpha, \quad j_{L P G E, y}=\left(\chi_{+}+\chi_{-} \cos 2 \alpha\right) \hat{e}_{y} E_{0}^{2},
$$

where $\chi_{ \pm}=\left(\chi_{x x z} \pm \chi_{y y z}\right) / 2$. In the experimental set-up used for the measurement of the helicity dependence of the spin photocurrents, the laser light is linearly polarized along $x$ and a $\lambda / 4$ plate is placed between the laser and the sample; equations (40) take the form

$$
j_{L P G E, x}=\chi_{x x z} \hat{e}_{y} E_{0}^{2} \cos 2 \varphi \sin 2 \varphi, \quad j_{L P G E, y}=\left(\chi_{+}+\chi_{-} \cos 2 \varphi\right) \hat{e}_{y} E_{0}^{2} .
$$

In addition to the current given by equations (40), the point group $\mathrm{C}_{\mathrm{s}}$ allows a LPGE current for normal incidence of the radiation, because in this case the tensor $\chi$ has the additional nonzero components $\chi_{x x y^{\prime}}=\chi_{x y^{\prime} x}, \chi_{y^{\prime} x x}$, and $\chi_{y^{\prime} y^{\prime} y^{\prime}}$. This current is given by

$$
j_{L P G E, x}=\chi_{x x y^{\prime}}\left(E_{x} E_{y^{\prime}}^{*}+E_{y^{\prime}} E_{x}^{*}\right), \quad j_{L P G E, y^{\prime}}=\left(\chi_{y^{\prime} x x}\left|E_{x}\right|^{2}+\chi_{y^{\prime} y^{\prime} y^{\prime}}\left|E_{y^{\prime}}\right|^{2}\right)
$$

yielding for linearly polarized light

$$
j_{L P G E, x}=\chi_{x x y^{\prime}} \hat{e}_{z^{\prime}} E_{0}^{2} \sin 2 \alpha, \quad j_{L P G E, y^{\prime}}=\left(\chi_{+}^{\prime}+\chi_{-}^{\prime} \cos 2 \alpha\right) \hat{e}_{z^{\prime}} E_{0}^{2},
$$

where $\chi_{ \pm}^{\prime}=\left(\chi_{x x x} \pm \chi_{x y^{\prime} y^{\prime}}\right) / 2$, and, for the experimental arrangement of the helicity dependence measurements,

$j_{L P G E, x}=\chi_{x x y^{\prime}} \hat{e}_{z^{\prime}} E_{0}^{2} \cos 2 \varphi \sin 2 \varphi, \quad j_{L P G E, y^{\prime}}=\left(\chi_{+}^{\prime}+\chi_{-}^{\prime} \cos 2 \varphi\right) \hat{e}_{z^{\prime}} E_{0}^{2}$,

The dependence of the LPGE current on the angle of incidence $\Theta_{0}$ is determined by the projections of the unit vector $\hat{e}$ which, as in the case of the CPGE, have the forms of equations (16)-(18).

Equations (42) and (45) for the LPGE under excitation with elliptically polarized radiation show that it may occur simultaneously with spin orientation-induced CPGE (see equations (14) and (15)). However, in this case the LPGE is equal to zero for circularly polarized radiation for all symmetries considered. Indeed, light is circularly polarized at $2 \varphi=\pi / 2,3 \pi / 2, \ldots$; therefore for $\sigma_{ \pm}$-radiation, $\cos 2 \varphi$ in equations (42) and (45) is equal to zero and the LPGE current vanishes. For $P_{\text {circ }}$ between -1 and +1 , as in the measurements of the helicity dependence of the photocurrent, the spin orientation-induced CPGE and LPGE can be simultaneously present. However, measurements carried out on GaAs [10, 20-22], 


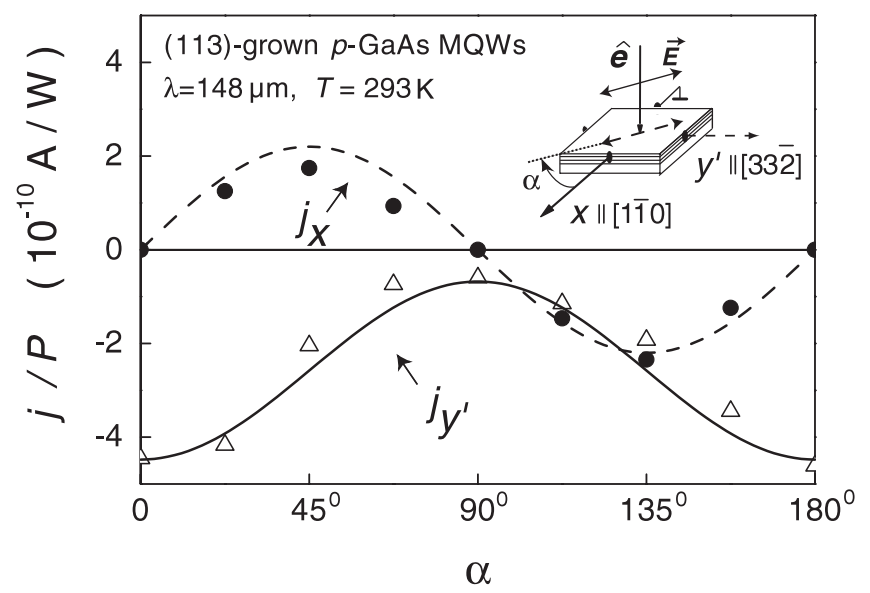

Figure 27. The linear photogalvanic current $j$ normalized by $P$ as a function of the angle $\alpha$ between the plane of linear polarization and the axis $x$. Data are obtained for the $x$-and $y^{\prime}$-directions under normal incidence. The broken curve and the full curve were fitted using equations (44).

InAs [20, 21], and ZnSeMnTe [43] QWs over the whole wavelength range as well as on SiGe QWs in the mid-infrared [23, 107] have shown that the contribution of the LPGE is negligible (see figures 10 and 13).

The situation is different for SiGe QWs under FIR excitation where a spin orientationinduced CPGE and LPGE with comparable strength have been detected [23, 107]. Figure 15 exhibits experimental data obtained on p-type SiGe (113)-grown QW structures. Broken and dotted curves show the contributions of the circular photocurrent, $j_{x} \propto \sin 2 \varphi$, and the linear photocurrent, $j_{x} \propto \sin 2 \varphi \cos 2 \varphi$, respectively. The tensors $\gamma$ and $\chi$ describe different physical mechanisms and, therefore, may depend differently on the material parameters, excitation wavelength, and temperature. Obviously in most cases the contribution of $\gamma$ to the photocurrent is larger than that of $\chi$.

The occurrence of a LPGE without a CPGE is demonstrated by measurements for linear polarization, where the CPGE is zero, depicted in figure 27 for GaAs QWs and in figure 28 for SiGe QWs. It was also observed under excitation with elliptically polarized radiation with $P_{\text {circ }}$ varying from -1 to +1 in a geometrical direction where the CPGE is forbidden by symmetry. This is demonstrated in figure 29 where a longitudinal current generated with oblique incidence along the [110] direction is shown.

\subsection{The photon drag effect}

The photon drag effect arises due to a momentum transfer from photons to free carriers. This effect was first observed in bulk semiconductors $[130,131]$ and has been investigated in various materials such as $\mathrm{Ge}, \mathrm{Si}$, and $\mathrm{GaP}$ for a wide range of optical excitation mechanisms such as inter-band transitions, direct and indirect transitions in free carrier absorption, and impurity ionization (for a review see [118, 119]). It was also intensively studied for GaAs and InAs QWs [21, 132-139]. The photon drag is of great technical importance for fast infrared and FIR detection of short laser pulses [104, 105, 118, 119, 140].

The photon drag current described by the second term of the right-hand side of equation (39) is mediated by a fourth-rank tensor $\boldsymbol{T}$. Therefore there is no symmetry restriction for this effect. In $T_{\lambda \delta \mu \nu}$ the first index $\lambda$ runs over $x$ and $y$ only, because the current must be confined in the plane of the QW. 


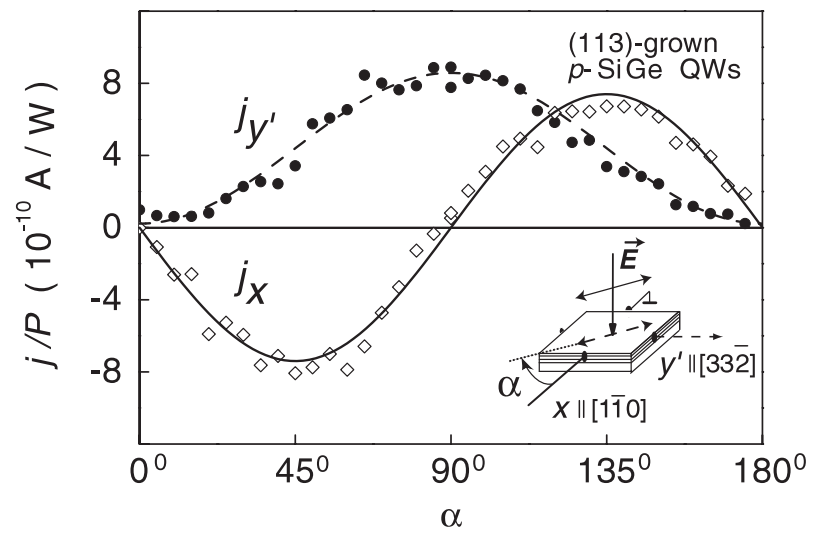

Figure 28. The linear photogalvanic current $j$ normalized by $P$ for the $x$ - and $y^{\prime}$-directions as a function of the angle $\alpha$ between the plane of linear polarization and the axis $x$. The results were obtained at room temperature with (113)-grown SiGe QWs for normal incidence of irradiation at $\lambda=280 \mu \mathrm{m}$. The broken curve and the full curve were fitted using equations (44).

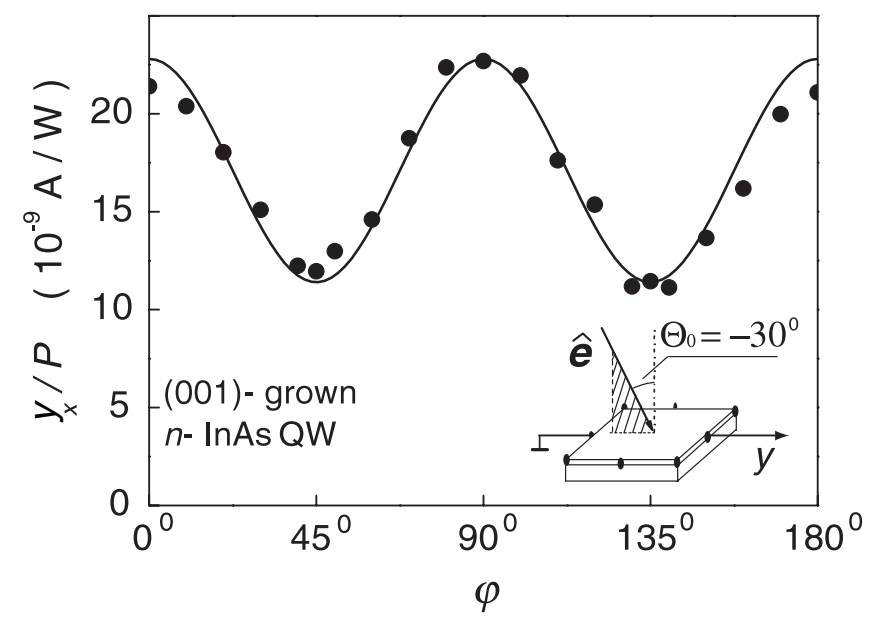

Figure 29. The linear photogalvanic current $j_{y}$ normalized by $P$ as a function of the phase angle $\varphi$ measured at $I=293 \mathrm{~K}$ and $\lambda=76 \mu \mathrm{m}$. The solid curve was fitted using equation (42).

For QWs of $\mathrm{C}_{2 \mathrm{v}}$ symmetry the photon drag effect yields the current

$$
\begin{aligned}
& j_{P D, x}=\sum_{\mu=x, y, z} T_{x x \mu \mu} q_{x}\left|E_{\mu}\right|^{2}+T_{x y x y} q_{y}\left(E_{x} E_{y}^{*}+E_{y} E_{x}^{*}\right), \\
& j_{P D, y}=\sum_{\mu=x, y, z} T_{y y \mu \mu} q_{y}\left|E_{\mu}\right|^{2}+T_{y x y x} q_{x}\left(E_{x} E_{y}^{*}+E_{y} E_{x}^{*}\right) .
\end{aligned}
$$

For $\mathrm{C}_{\mathrm{s}}$ symmetry this equation has the same form if $y$ and $z$ are replaced by the primed coordinates. In more highly symmetric QWs of point group $\mathrm{D}_{2 \mathrm{~d}}$, the number of independent non-zero tensor components is reduced by

$$
T_{x x x x}=T_{y y y y}, \quad T_{x x y y}=T_{y y x x}, \quad T_{x x z z}=T_{y y z z}, \quad T_{x y x y}=T_{y x y x} .
$$

The above equations show that the photon drag effect occurs in QWs of all symmetries for oblique incidence of radiation only. The longitudinal effect-the first term on the righthand side of equations (46) — is usually much stronger than the transverse effect described by 
the second term. This is the reason that in the transverse geometry of the spin orientationinduced CPGE, no influence of the photon drag effect could be detected as yet. However, in the longitudinal geometry the photon drag effect yields a measurable current. Again helicity dependence can help one to distinguish spin photocurrents from the photon drag effect. On the other hand, the separation of the LPGE and the photon drag effect is not so obvious. The usual method used to identify the photon drag effect is based on sign inversion of the current by reversing the wavevector of light in the plane of the sample. The same sign inversion occurs also for the LPGE (see equations (16), (18), (41), and (42)).

Both effects may be distinguished from polarization dependences. The situation is simple for transverse currents. Indeed, the LPGE vanishes for linearly polarized radiation with the radiation electric field normal or parallel to the current flow (see the left-hand parts of equations (41), (44)) or for circularly polarized radiation (see the left-hand parts of equations (42), (45)), whereas a photon drag effect may be present (see equations (46)). However, for longitudinal currents the photon drag effect and the LPGE may be present at the same time with comparable strengths for any polarization. In contrast to the transverse effect, the longitudinal LPGE has a polarization-independent term $\chi_{+}$in the right-hand parts of equations (41), (42) and $\chi_{+}^{\prime}$ in the right-hand parts of equations (44), (45). Thus, a longitudinal current in non-centrosymmetric QWs which changes sign upon reversal of the light propagation need not be a photon drag current. The characteristic polarization dependences as well as the helicity dependence may help in identifying the underlying microscopic mechanisms. We note that by investigation of the photon drag effect in QWs without inversion centres, one should always take into account the LPGE contribution and vice versa. For elliptical polarization, the spin orientation-induced CPGE may also contribute to the total current.

As a concluding remark, spin photocurrents can in any case be distinguished from helicityindependent currents by switching the helicity from right to left or the other way round. The fraction of spin photocurrents in the total current can be quantitatively extracted by modulation methods.

\section{Spin photocurrents caused by inhomogeneities}

One of the essential features of spin photocurrents reviewed here is the homogeneity of both the optical excitation and the distribution of spin polarization in a two-dimensional electron gas of gyrotropic QWs. However, spin photocurrents may occur due to an inhomogeneous spin distribution obtained by inhomogeneous optical excitation or in bulk inhomogeneities such as in $\mathrm{p}-\mathrm{n}$ junctions.

As was already introduced, a surface current $j=e \zeta$ rot $S$ due to inhomogeneity of the spin polarization $S$ of electrons at a semiconductor surface layer proposed in [7] was observed in bulk AlGaAs samples [9]. Here $\zeta$ is a coefficient proportional to the energy of the spin-orbit splitting of the valence band $\Delta_{s o}$. The inhomogeneous spin polarization was obtained by the strong absorption of circularly polarized radiation at the band edge of AlGaAs mixed crystals (HeNe laser excitation). The radiation was normally incident on the sample, resulting in a gradient of the spin density into the material due to surface generation and diffusion into the bulk. Spin-orbit interaction at the surface yields asymmetric electron scattering which gives rise to a current in the direction perpendicular to the gradient of the spin density and the average electron spin. An external magnetic field oriented in the surface plane was used to optimize the current by rotating the spin polarization into the surface plane (see figure 30(a)) [9]. The surface current exhibits the Hanle effect as a function of the magnetic field strength. The geometry and the experimental procedure are very similar to those used to demonstrate the spin-galvanic effect (see figure 7). The crucial difference from the case of the spin-galvanic 
(a) He-Ne laser light

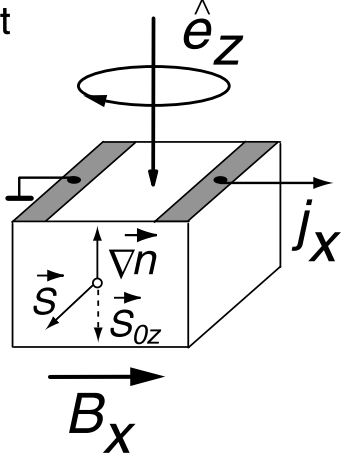

(b) visible light

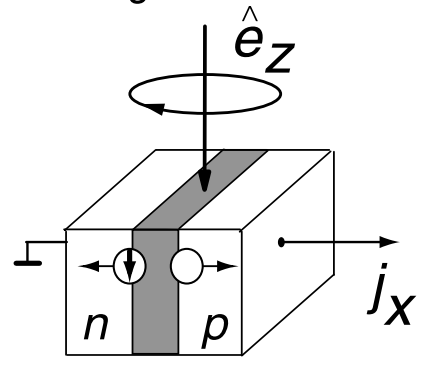

Figure 30. Sketches of the experimental arrangement of: (a) the surface spin photocurrent due to non-homogeneous spin orientation following [9], where $\nabla \boldsymbol{n}$ indicates the gradient of the electron density in the penetration depth of the strong fundamental absorption; (b) the spin-voltaic effect according to [141, 142], where the circle with an arrow indicates spin-polarized electrons and the open circle represents unpolarized holes due to rapid spin relaxation.

effect is that here, for a surface photocurrent caused by optical orientation, a gradient of spin density is needed. Naturally this gradient is absent in QWs where the spin-galvanic effect has been investigated, because QWs are two dimensional (no 'thickness').

Another type of spin photocurrents was recently proposed in analogy to the photovoltaic effect in $\mathrm{p}-\mathrm{n}$ junctions $[141,142]$. This spin-voltaic effect occurs due to uniform illumination of a $\mathrm{p}-\mathrm{n}$ junction with circularly polarized inter-band light resulting in spin polarization of a charge current. Circular polarization generates spin-polarized electrons and holes. Due to the fast relaxation of the hole spin polarization in the bulk and the long spin lifetime of electrons, the photocurrent becomes spin polarized. Indeed, spin-polarized electrons are swept to the $n$ side by the built-in electric field $E_{b i}$ and the unpolarized holes drift to the p side of the junction (see figure 30(b)).

\section{Summary}

A non-equilibrium uniform spin polarization obtained by optical orientation drives an electric current in QWs if they belong to a gyrotropic crystal class. In QWs prepared from zinc-blende structure materials, gyrotropy is naturally present due to the lack of inversion symmetry in the basic material which is itself not gyrotropic. In QWs based on diamond structure materials, such as $\mathrm{Si}$ and $\mathrm{Ge}$, which possess a centre of inversion, gyrotropy may be introduced by artificially growing asymmetric structures. In gyrotropic QWs, spin-orbit interaction results in a spin splitting in $\boldsymbol{k}$-space of subbands, yielding the basis of spin photocurrents. Two different microscopic mechanisms for spin photocurrents can be distinguished: the spin orientationinduced CPGE and the spin-galvanic effect. In the first effect the coupling of the helicity of light to spin-polarized final states with a net linear momentum is caused by angular momentum selection rules together with band splitting in $\boldsymbol{k}$-space due to $\boldsymbol{k}$-linear terms in the Hamiltonian. The current flow in the second effect is driven by asymmetric spin relaxation of a homogeneous non-equilibrium spin polarization. The current is present even if the initial electron distribution in each spin-split subband is uniform.

The experimental results on spin photocurrents due to homogeneous spin polarization are in good agreement with the phenomenological theory. Both mechanisms of spin 
photocurrents, as well as the removal of the spin degeneracy in $k$-space, are described by second-rank pseudo-tensors. Because of the tensor equivalence in the different symmetries, the irreducible components of these tensors differ by scalar factors only. Therefore, macroscopic measurements of photocurrents in different geometric configurations of experiments allow one to reach a conclusion as regards the details of the microscopic tensorial spin-orbit interaction. In particular, the relation between the symmetric and anti-symmetric parts of the spin-orbit interaction, representing Dresselhaus-like terms (including IIA) and the Rashba term, respectively, may be obtained. Furthermore, the macroscopic symmetry of QWs may easily be determined.

Spin photocurrents are obtained from circularly polarized radiation. The most important feature of spin photocurrents is their helicity dependence. The current is proportional to the helicity and reverses its direction upon the handedness of the radiation changing. The effect is a quite general property of QWs and has already been observed in many different n- and p-type semiconductor structures under various kinds of optical excitation such as inter-band and free carrier absorption. It is present in materials of different mobilities, and also at very low mobility, for a wide range of carrier densities and can be detected even at room temperature. Spin photocurrents are not limited to 2D structures. Most recently they have been predicted for gyrotropic 1D systems such as carbon nanotubes of spiral symmetry [143]. The effect is caused by coupling between the electron wavevector along the tube principal axis and the orbital momentum around the tube circumference.

Spin photocurrents were used to investigate the mechanism of spin relaxation for monopolar spin orientation where only one type of charge carrier is involved in the excitationrelaxation process. This condition is close to that for electrical spin injection in semiconductors. Two methods were applied to determine spin relaxation times: those based on the Hanle effect in the spin-galvanic current and the spin-sensitive bleaching of photogalvanic currents. The spin orientation-induced CPGE has also been used to detect the state of polarization of terahertz radiation [42]. The rapid momentum relaxation at room temperature in QWs yields picosecond time resolution.

\section{Acknowledgments}

The authors thank E L Ivchenko, V V Bel'kov, L E Golub, S N Danilov, and P Schneider for many discussions and helpful comments on the present work. We are also indebted to G Abstreiter, V V Bel'kov, M Bichler, J DeBoeck, G Borghs, K Brunner, S N Danilov, J Eroms, E L Ivchenko, S Giglberger, P Grabs, L E Golub, T Humbs, J Kainz, H Ketterl, B N Murdin, P Schneider, D Schuh, M Sollinger, S A Tarasenko, L Molenkamp, R Neumann, V I Perel, C R Pidgeon, P J Phillips, U Rössler, W Schoepe, D Schowalter, G Schmidt, V M Ustinov, L E Vorobjev, D Weiss, W Wegscheider, D R Yakovlev, I N Yassievich, and A E Zhukov for long-standing cooperation during the work on spin photocurrents. We gratefully acknowledge financial support by the Deutsche Forschungsgemeinschaft (DFG), the Russian Foundation for Basic Research (RFBR) and the NATO Linkage Grant which made this work possible.

\section{References}

[1] Awschalom D D, Loss D and Samarth N (ed) 2002 Semiconductor Spintronics and Quantum Computation (Nanoscience and Technology Series) series ed K von Klitzing, H Sakaki and R Wiesendanger (Berlin: Springer)

[2] Bychkov Y A and Rashba E I 1984 Pis. Zh. Eksp. Teor. Fiz. 3966 (Engl. transl. 1984 Sov. Phys.-JETP 39 78)

[3] Meier F and Zakharchenya B P (ed) 1984 Optical Orientation (Amsterdam: Elsevier)

[4] Hägele D, Oestreich M, Rühle W W, Nestle N and Eberl K 1998 Appl. Phys. Lett. 731580

[5] Kikkawa J M and Awschalom D D 1999 Nature 397139 
[6] Hirohata A, Xu Y B, Guertler C M and Bland A C 1999 J. Appl. Phys. 855804

[7] Averkiev N S and D'yakonov M I 1983 Fiz. Tekh. Poluprov. 17629 (Engl. transl. 1983 Sov. Phys.-Semicond. 17 393)

[8] D’yakonov M I and Perel' V I 1971 Pis. Zh. Eksp. Teor. Fiz. 13206 (Engl. transl. 1971 Sov. Phys.-JETP 13 144)

[9] Bakun A A, Zakharchenya B P, Rogachev A A, Tkachuk M N and Fleisher V G 1984 Pis. Zh. Eksp. Teor. Fiz. 40464 (Engl. transl. 1984 Sov. Phys.-JETP 40 1293)

[10] Ganichev S D, Ivchenko E L, Ketterl H, Prettl W and Vorobjev L E 2000 Appl. Phys. Lett. 773146

[11] Ivchenko E L and Pikus G E 1980 Problems of Modern Physics ed V M Tuchkevich and V Ya Frenkel (Leningrad: Nauka) p 275 (Engl. transl. 1986 Semiconductor Physics ed V M Tuchkevich and V Ya Frenkel (New York: Consultants Bureau) p 427)

[12] Belinicher V I and Sturman B I 1980 Usp. Fiz. Nauk 130415 (Engl. transl. 1980 Sov. Phys.-Usp. 23 199)

[13] Sturman B I and Fridkin V M 1992 The Photovoltaic and Photorefractive Effects in Non-Centrosymmetric Materials (New York: Gordon and Breach)

[14] Ivchenko E L and Pikus G E 1997 Superlattices and Other Heterostructures. Symmetry and Optical Phenomena (Berlin: Springer)

[15] Ivchenko E L and Pikus G E 1978 Pis. Zh. Eksp. Teor. Fiz. 27640 (Engl. transl. 1978 Sov. Phys.-JETP 27 604)

[16] Belinicher V I 1978 Phys. Lett. A 66213

[17] Asnin V M, Bakun A A, Danishevskii A M, Ivchenko E L, Pikus G E and Rogachev A A 1978 Pis. Zh. Eksp. Teor. Fiz. 2880 (Engl. transl. 1978 Sov. Phys.-JETP 28 74)

[18] Asnin V M, Bakun A A, Danishevskii A M, Ivchenko E L, Pikus G E and Rogachev A A 1979 Solid State Commun. 30565

[19] Averkiev N S, Asnin V M, Bakun A A, Danishevskii A M, Ivchenko E L, Pikus G E and Rogachev A A 1984 Fiz. Tekh. Poluprov. 18639 (Engl. transl. 1984 Sov. Phys.-Semicond. 18 397)

Averkiev N S, Asnin V M, Bakun A A, Danishevskii A M, Ivchenko E L, Pikus G E and Rogachev A A 1984 Fiz. Tekh. Poluprov. 18648 (Engl. transl. 1984 Sov. Phys.-Semicond. 18 402)

[20] Ganichev S D, Ivchenko E L, Danilov S N, Eroms J, Wegscheider W, Weiss D and Prettl W 2001 Phys. Rev. Lett. 864358

[21] Ganichev S D, Ivchenko E L and Prettl W 2002 Physica E 14166

[22] Ganichev S D 2003 Proc. ICPS-26 at press

[23] Ganichev S D, Rössler U, Prettl W, Ivchenko E L, Bel'kov V V, Neumann R, Brunner K and Abstreiter G 2002 Phys. Rev. B 66075328

[24] Ganichev S D, Bel'kov V V, Schneider P, Ivchenko E L, Tarasenko S A, Schuh D, Wegscheider W, Weiss D and Prettl W 2003 Phys. Rev. B submitted

(Ganichev S D, Bel'kov V V, Schneider P, Ivchenko E L, Tarasenko S A, Schuh D, Wegscheider W, Weiss D and Prettl W 2003 Preprint cond-mat/0303054)

[25] Golub L E 2003 Physica E at press (Golub L E 2002 Preprint cond-mat/0208295)

[26] Golub L E 2003 Phys. Rev. B at press

[27] Ivchenko E L, Lyanda-Geller Yu B and Pikus G E 1989 Pis. Zh. Eksp. Teor. Fiz. 50156 (Engl. transl. 1989 Sov. Phys.-JETP 50 175)

[28] Ivchenko E L, Lyanda-Geller Yu B and Pikus G E 1990 Zh. Eksp. Teor. Fiz. 98989 (Engl. transl. 1990 Sov. Phys.-JETP 71 550)

[29] Ganichev S D, Ivchenko E L, Bel'kov V V, Tarasenko S A, Sollinger M, Weiss D, Wegscheider W and Prettl W 2002 Nature 417153

[30] Ganichev S D, Ivchenko E L, Bel'kov V V, Tarasenko S A, Sollinger M, Schowalter D, Weiss D, Wegscheider W and Prettl W 2003 J. Supercond.: Incorp. Novel Magn. 16369 (Preprint cond-mat/0301390)

[31] Ganichev S D, Schneider P, Bel'kov V V, Ivchenko E L, Tarasenko S A, Wegscheider W, Weiss D, Schuh D, Clarke D G, Merrick M, Murdin B N, Murzyn P, Phillips P J, Pidgeon C R, Beregulin E V and Prettl W 2003 Phys. Rev. B submitted

(Ganichev S D, Schneider P, Bel'kov V V, Ivchenko E L, Tarasenko S A, Wegscheider W, Weiss D, Schuh D, Clarke D G, Merrick M, Murdin B N, Murzyn P, Phillips P J, Pidgeon C R, Beregulin E V and Prettl W 2003 Preprint cond-mat/0303193)

[32] Bhat R D R and Sipe J E 2000 Phys. Rev. Lett. 855432

[33] Stevens M J, Smirl A L, Bhat R D R, Sipe J E and van Driel H M 2002 J. Appl. Phys. 914382

[34] Entin M V 1989 Fiz. Tekh. Poluprov. 231066 (Engl. transl. 1989 Sov. Phys.-Semicond. 23 664)

[35] Magarill L I 2001 Physica E 9652

[36] Ganichev S D, Ketterl H and Prettl W 1999 Physica B 272464 
[37] Ganichev S D, Bel'kov V V, Danilov S N, Ivchenko E L, Ketterl H, Vorobjev L E, Bichler M, Wegscheider W and Prettl W 2001 Physica E 1052

[38] Ganichev S D, Danilov S N, Bel'kov V V, Ivchenko E L, Bichler M, Wegscheider W, Weiss D and Prettl W 2002 Phys. Rev. Lett. 88 057401-1

[39] Ganichev S D, Danilov S N, Sollinger M, Weiss D, Wegscheider W, Prettl W, Bel'kov V V and Ivchenko E L 2001 MRS Symp. Proc. vol 690, ed T J Klemmer, J S Sun, A Fert and J Bass (Pittsburgh, PA: Materials Research Society) p F1.2.1

[40] Tarasenko S A, Ivchenko E L, Bel'kov V V, Ganichev S D, Schowalter D, Schneider P, Sollinger M, Prettl W, Ustinov V M, Zhukov A E and Vorobjev L E 2003 Proc. ICPS-26 at press

(Tarasenko S A, Ivchenko E L, Bel'kov V V, Ganichev S D, Schowalter D, Schneider P, Sollinger M, Prettl W, Ustinov V M, Zhukov A E and Vorobjev L E 2003 Preprint cond-mat/0301393)

[41] Tarasenko S A, Ivchenko E L, Bel'kov V V, Ganichev S D, Schowalter D, Schneider P, Sollinger M, Prettl W, Ustinov V M, Zhukov A E and Vorobjev L E 2003 J. Supercond.: Incorp. Novel Magn. 16419 (Preprint cond-mat/0301388)

[42] Ganichev S D, Ketterl H and Prettl W 2003 Int. J. Infrared Millimetre Waves 24

[43] Ganichev S D, Sollinger M, Prettl W, Yakovlev D R, Grabs P, Schmidt G, Molenkamp L and Ivchenko E L 2001 Ver. Deutsch. Phys. Ges. V 361170

[44] Stein D, von Klitzing K and Weimann G 1983 Phys. Rev. Lett. 51130

[45] Stormer H L, Schlesinger Z, Chang A, Tsui D C, Gossard A C and Wiegmann W 1983 Phys. Rev. Lett. 51126

[46] D’yakonov M I and Kachorovskii V Yu 1986 Fiz. Tekh. Poluprov. 20178 (Engl. transl. 1986 Sov. Phys.Semicond. 20 110)

[47] Averkiev N S, Golub L E and Willander M 2002 J. Phys.: Condens. Matter 14 R271

[48] Voskoboynikov A, Liu S S and Lee C P 1998 Phys. Rev. B 5815397

[49] de Andrada e Silva E A and La Rocca G C 1999 Phys. Rev. B 5915583

[50] Koga T, Nitta J, Takayanagi H and Datta S 2002 Phys. Rev. Lett. 88126601

[51] Perel V I, Tarasenko S A, Yassievich I N, Ganichev S D, Bel'kov V V and Prettl W 2003 Phys. Rev. B submitted (Perel V I, Tarasenko S A, Yassievich I N, Ganichev S D, Bel'kov V V and Prettl W 2003 Preprint condmat/0301098)

[52] Kalevich V K and Korenev V L 1990 Pis. Zh. Eksp. Teor. Fiz. 52859 (Engl. transl 1990 Sov. Phys.-JETP 52 230)

[53] Nitta J, Akazaki T and Takayanagi H 1997 Phys. Rev. Lett. 781335

[54] Lu J P, Yau J B, Shukla S P, Shayegan M, Wissinger L, Rössler U and Winkler R 1998 Phys. Rev. Lett. 81 1282

[55] Heida J P, van Wees B J, Kuipers J J, Klapwijk T M and Borghs G 1998 Phys. Rev. B 5711911

[56] Hu C-M, Nitta J, Akazaki T, Takayanagi H, Osaka J, Pfeffer P and Zawadski W 1999 Phys. Rev. B 607736

[57] Salis G, Kato Y, Ensslin K, Driscoll D C, Gossard A C and Awschalom D D 2001 Nature 414619

[58] Smet J H, Deutschmann R A, Ertl F, Wegscheider W, Abstreiter G and von Klitzing K 2002 Nature 415281

[59] Vas'ko F T and Prima N A 1979 Fiz. Tverd. Tela 211734 (Engl. transl. 1979 Sov. Phys.-Solid State 21 994)

[60] Rössler U 1984 Solid State Commun. 49943

[61] Cardona M, Christensen N E and Fasol G 1988 Phys. Rev. B 381806

[62] Lommer G, Malcher F and Rössler U 1988 Phys. Rev. Lett. 60728

[63] Luo J, Munekata H, Fang F F and Stiles P J 1988 Phys. Rev. B 3810142

[64] Das B, Miller D C, Datta S, Reifenberger R, Hong W P, Bhattacharya P K, Singh J and Jaffe M 1989 Phys. Rev. B 391411

[65] Luo J, Munekata H, Fang F F and Stiles P J 1990 Phys. Rev. B 417685

[66] Ivanov Yu L, Kop'ev P S, Suchalkin S D and Ustinov V M 1991 Pis. Zh. Eksp. Teor. Phys. 53470 (Engl. transl. 1991 Sov. Phys.-JETP 53 493)

[67] Dresselhaus P D, Papavassiliou C M A, Wheeler R G and Sacks R N 1992 Phys. Rev. Lett. 68106

[68] de Andrada e Silva E A 1992 Phys. Rev. B 461921

[69] Santos R V and Cardona M 1994 Phys. Rev. Lett. 72432

[70] Andrada e Silva E A, La Rocca G C and Bassani F 1994 Phys. Rev. B 508523

[71] Jusserand B, Richards D, Allan G, Priester C and Etienne B 1995 Phys. Rev. B 514707

[72] Pfeffer P and Zawadski W 1995 Phys. Rev. B 52 R14332

[73] Ivchenko E L, Kaminski A Yu and Rössler U 1996 Phys. Rev. B 545852

[74] Engels G, Lange J, Schäpers Th and Lüth H 1997 Phys. Rev. B 55 R1958

[75] Andrada e Silva E A, La Rocca G C and Bassani F 1997 Phys. Rev. B 5516293

[76] Grundler D 2000 Phys. Rev. Lett. 846074

[77] Hammar P R and Johnson M 2000 Phys. Rev. B 617207 
[78] Majewski J A, Vogl P and Lugli P 2001 Proc. ICPS: 25th Int. Conf. on Physics of Semiconductors ed N Miura and T Ando (Berlin: Springer) p 791

[79] Wilamowski Z, Jantsch W, Malissa H and Rössler U 2002 Phys. Rev. B 66195315

[80] Tarasenko S A and Averkiev N S 2002 Pis. Zh. Eksp. Teor. Fiz. 75669 (Engl. transl. 2002 Sov. Phys.-JETP 75 552)

[81] Krebs O and Voisin P 1996 Phys. Rev. Lett. 771829

[82] Guettler T, Triques A L C, Vervoort L, Ferreira R, Roussignol Ph, Voisin P, Rondi D and Harmand J C 1998 Phys. Rev. B 58 R 10179

[83] Krebs O, Rondi D, Gentner J L, Goldstein L and Voisin P 1998 Phys. Rev. Lett. 805770

[84] Toropov A A, Ivchenko E L, Krebs O, Cortez S, Voisin P and Gentner J L 2000 Phys. Rev. B 63035302

[85] Olesberg J T, Lau W H, Flatte M, Altunkaya C Yu E, Shaw E M, Hasenberg T C and Bogges T 2001 Phys. Rev. B 64201301

[86] Rössler U and Kainz J 2002 Solid State Commun. 121313

[87] Golub L E and Ivchenko E L 2003 at press

(Golub L E and Ivchenko E L 2003 Preprint cond-mat/0302308)

[88] Dresselhaus G 1955 Phys. Rev. 100580

[89] Rashba E I 1960 Fiz. Tverd. Tela 21224 (Engl. transl. 1960 Sov. Phys.-Solid State 2 1109)

[90] Datta S and Das B 1990 Appl. Phys. Lett. 56665

[91] Ivchenko E L and Tarasenko S A 2003 JETP at press

[92] Haché A, Kostoulas Y, Atanasov R, Hudges J L P, Sipe J E and van Driel H M 1997 Phys. Rev. Lett. 78306

[93] Aronov A G and Lyanda-Geller Yu B 1989 Pis. Zh. Eksp. Teor. Phys. 50398 (Engl. transl. 1990 Sov. Phys.-JETP 50 431)

[94] Edelstein V M 1990 Solid State Commun. 73233

[95] Parson R R 1971 Can. J. Phys. 491850

[96] Hanle W 1924 Z. Phys. 3093

[97] Andrianov A V and Yaroshetskii I D 1984 Pis. Zh. Eksp. Teor. Fiz. 40131 (Engl. transl. 1984 Sov. Phys.-JETP 40 882)

[98] Ivchenko E L, Lyanda-Geller Yu B and Pikus G E 1989 Solid State Commun. 69663

[99] Behet M, Nemeth S, DeBoeck J, Borghs G, Tümmler J, Woitok J and Geurts J 1998 Semicond. Sci. Technol. 13428

[100] Fiederling R, Keim M, Reuscher G, Ossau W, Schmidt G, Waag A and Molenkamp L W 1999 Nature 402787

[101] Ganichev S D 1999 Physica B 273/274 737

[102] Ganichev S D, Yassievich I N and Prettl W 2002 J. Phys.: Condens. Matter 14 R1263

[103] Knippels G M H, Yan X, MacLeod A M, Gillespie W A, Yasumoto M, Oepts D and van der Meer A F G 1999 Phys. Rev. Lett. 831578

[104] Ganichev S D, Terent'ev Ya V and Yaroshetskii I D 1985 Pis. Zh. Tekh. Phys. 1146 (Engl. transl. 1985 Sov. Tech. Phys. Lett. 11 20)

[105] Beregulin E V, Ganichev S D, Yaroshetskii I D, Lang P T, Schatz W and Renk K F 1990 Proc. SPIE vol 1362-2, ed M Razeghi (Bellingham, WA: SPIE Optical Engineering Press) p 853

[106] Warburton R J, Gauer C, Wixforth A, Kotthaus J P, Brar B and Kroemer H 1996 Phys. Rev. B 537903

[107] Bel'kov V V, Ganichev S D, Schneider P, Schowalter D, Rössler U, Prettl W, Ivchenko E L, Neumann R, Brunner K and Abstreiter G 2003 J. Supercond.: Incorp. Novel Magn. 16419 (Preprint cond-mat/0301389)

[108] Marie X, Amand T, Le Jeune P, Paillard M, Renucci P, Golub L E, Dymnikov V D and Ivchenko E L 1999 Phys. Rev. B 605811

[109] Lampel G 1968 Phys. Rev. Lett. 20491

[110] Ekimov A I and Safarov V I 1970 Pis. Zh. Eksp. Teor. Fiz. 12293 (Engl. transl. 1970 Sov. Phys.-JETP 12 198)

[111] Zakharchenya B I, Fleisher V G, Dzhioev R I, Veshchunov Yu P and Rusanov I B 1971 Pis. Zh. Eksp. Teor. Fiz. 13195 (Engl. transl. 1971 Sov. Phys.-JETP 13 137)

[112] Danishevskii A M, Ivchenko E L, Kochegarov S F and Subashiev V K 1985 Fiz. Tverd. Tela 27710 (Engl. transl. 1985 Sov. Phys.-Solid State 27 439)

[113] Ferreira R and Bastard G 1991 Phys. Rev. B 439687

[114] Vorobjev L E, Donetskii D V and Golub L E 1996 Pis. Zh. Eksp. Teor. Fiz. 63977 (Engl. transl. 1996 Sov. Phys.-JETP 63 981)

[115] Schneider P, Ganichev S D, Kainz J, Rössler U, Wegscheider W, Weiss D, Prettl W, Bel'kov V V, Golub L E and Schuh D 2003 Phys. Status Solidi a at press

(Schneider P, Ganichev S D, Kainz J, Rössler U, Wegscheider W, Weiss D, Prettl W, Bel'kov V V, Golub L E and Schuh D 2003 Preprint cond-mat/0303056)

[116] Shah J 1999 Ultrafast Spectroscopy of Semiconductor Nanostructures (Berlin: Springer) pp 243-61 
[117] Viña L 1999 J. Phys.: Condens. Matter 115929

[118] Yaroshetskii I D and Ryvkin S M 1980 Problems of Modern Physics ed V M Tuchkevich and V Ya Frenkel (Leningrad: Nauka) p 173 (Engl. transl. 1986 Semiconductor Physics ed V M Tuchkevich and V Ya Frenkel (New York: Consultants Bureau) p 249)

[119] Gibson A F and Kimmitt M F 1980 Photon drag detection Infrared and Millimetre Waves vol 3, ed K J Button (New York: Academic) p 182

[120] Glass A M, von der Linde D and Negran T J 1974 Appl. Phys. Lett. 25233

[121] Belinicher V I, Malinovskii V K and Sturman B I 1977 Zh. Eksp. Teor. Fiz. 73692 (Engl. transl. 1977 Sov. Phys.-JETP 46 362)

[122] Magarill L I and Entin M V 1982 Poverchnost 174 (Engl. transl. 1982 Sov. Surf. 1 74)

[123] Gusev G M, Kvon Z D, Magarill L I, Palkin A M, Sozinov V I, Shegai O A and Entin M V 1987 Pis. Zh. Eksp. Teor. Fiz. 4628 (Engl. transl. 1987 Sov. Phys.-JETP 46 33)

[124] Schneider H, Ehret S, Schönbein C, Schwarz K, Bihlmann G, Fleissner J, Tränkle G and Böhm G 1998 Superlatt. Microstruct. 231289

[125] von Baltz R and Kraut W 1980 Phys. Lett. A 79364

[126] Belinicher V I, Ivchenko E L and Sturman B I 1982 Zh. Eksp. Teor. Fiz. 83649 (Engl. transl. 1982 Sov. Phys.-JETP 56 359)

[127] Ivchenko E L, Lyanda-Geller Yu B and Pikus G E 1984 Fiz. Tekh. Poluprov. 1893 (Engl. transl. 1984 Sov. Phys.-Semicond. 18 55)

[128] Andrianov A V, Beregulin E V, Ganichev S D, Gloukh K Yu and Yaroshetskii I D 1988 Pis. Zh. Tekh. Fiz. 14 1326 (Engl. transl. 1988 Sov. Tech. Phys. Lett. 14 580)

[129] Schönbein C, Schneider H, Bihlmann G, Schwarz K and Koidl P 1995 Appl. Phys. Lett. 68973

[130] Danishevskii A M, Kastal'skii A A, Ryvkin S M and Yaroshetskii I D 1970 Zh. Eksp. Teor. Fiz. 58544 (Engl. transl. 1970 Sov. Phys.-JETP 31 292)

[131] Gibson A F, Kimmitt M F and Walker A C 1970 Appl. Phys. Lett. 1775

[132] Luryi S 1987 Phys. Rev. Lett. 582263

[133] Wieck A D, Sigg H and Ploog K 1990 Phys. Rev. Lett. 64463

[134] Grinberg A A and Luryi S 1988 Phys. Rev. B 3887

[135] Dmitriev A P, Emel'yanov S A, Ivanov S V, Kop'ev P S, Terent'ev Ya V and Yaroshetskii I D 1991 Pis. Zh. Eksp. Teor. Fiz. 54460 (Engl. transl. 1991 Sov. Phys.-JETP 54 462)

[136] Keller O 1993 Phys. Rev. B 484786

[137] Beregulin E V, Voronov P M, Ivanov S V, Kop'ev P S and Yaroshetskii I D 1994 Pis. Zh. Eksp. Teor. Fiz. 59 83 (Engl. transl. 1994 Sov. Phys.-JETP 59 85)

[138] Vasko F T 1996 Phys. Rev. B 539576

[139] Vasko F T and Keller O 1998 Phys. Rev. B 5815666

[140] Sigg H, Kwakermaak M H, Margotte B, Erni D, van Son P and Köhler K 1995 Appl. Phys. Lett. 672827

[141] Žutić I, Fabian J and Das Sarma S 2001 Appl. Phys. Lett. 791558

[142] Žutić I, Fabian J and Das Sarma S 2002 Phys. Rev. Lett. 88066603

[143] Ivchenko E L and Spivak B 2002 Phys. Rev. B 66155404 